\title{
Status and Future of Nuclear Matrix Elements for Neutrinoless Double-Beta Decay: A Review
}

\author{
Jonathan Engel* \\ Department of Physics and Astronomy, University of North Carolina, Chapel Hill, NC 27516-3255, USA \\ Javier Menéndez ${ }^{\dagger}$ \\ Department of Physics, University of Tokyo, Hongo, Tokyo 113-0033, Japan
}

(Dated: March 28, 2017)

\begin{abstract}
The nuclear matrix elements that govern the rate of neutrinoless double beta decay must be accurately calculated if experiments are to reach their full potential. Theorists have been working on the problem for a long time but have recently stepped up their efforts as ton-scale experiments have begun to look feasible. Here we review past and recent work on the matrix elements in a wide variety of nuclear models and discuss work that will be done in the near future. Ab initio nuclearstructure theory, which is developing rapidly, holds out hope of more accurate matrix elements with quantifiable error bars.
\end{abstract}

\section{CONTENTS}

I. Introduction

II. Significance of Double-Beta Decay

A. Neutrino Masses and Hierarchy

B. Neutrinoless Double-Beta Decay

1. Light-neutrino Exchange

2. New Physics Mechanisms

C. Importance of Nuclear Matrix Elements for Experiments

III. Nuclear Matrix Elements At Present

A. Shell Model

B. The QRPA and Some of Its Variants

C. Energy-Density Functional Theory and the Generator-Coordinate Method

D. The Interacting Boson Model

E. Other Approaches

F. Tests and Comparisons

IV. The $g_{A}$ Problem

A. Systematic Over-prediction of Single- $\beta$ and $2 \nu \beta \beta$ Matrix Elements

B. Possible Causes and Implications for Neutrinoless Double-Beta Decay

V. Improving Matrix Element Calculations in the Next Few Years

A. General Ideas

B. Improving Present Models

1. Extending Shell Model Configuration Spaces

2. Adding Correlations to the EDF and the IBM

3. Higher QRPA and the Overlap Problem
C. Ab Initio Approaches

1. Kinds of $A b$ Initio Calculations

2. Effective Operators for the Shell Model from Ab Initio Calculations

3. Multi-reference IMSRG 24

D. Assessing Error

25

1. Statistical Error

2. Systematic Error and $g_{A}$

VI. Summary and Prospects

Acknowledgments

References

\section{INTRODUCTION}

Neutrinos are the only neutral fermions we know to exist. They are thus the only known particles that may be Majorana fermions, that is, their own antiparticles. Because neutrinos are so light, the difference in behavior between Majorana neutrinos and Dirac neutrinos, which would be distinct from their antiparticles, is slight. The easiest way to determine which of the two possibilities nature has chosen - and it is far from easy - is to see whether certain nuclei undergo neutrinoless double-beta $(0 \nu \beta \beta)$ decay, a second-order weak-interaction process in which the parent nucleus decays into its daughter with two fewer neutrons and two more protons, while emitting two electrons but, crucially, no (anti)neutrinos.

Experiments to observe $0 \nu \beta \beta$ decay are becoming more and more sensitive, and international teams are trying to push the sensitivity to the point at which they can identify a few decay events per year in a ton of material [1-4]. The hope is to be sensitive enough to detect $0 \nu \beta \beta$ decay if neutrinos are indeed Majorana particles and their masses are arranged in a pattern known as the "inverted hierarchy" (discussed in Sec. II A). Because the decay takes place inside nuclei, the amount of material

\footnotetext{
* engelj@physics.unc.edu

† menendez@nt.phys.s.u-tokyo.ac.jp
} 
required to fully cover the inverted-hierarchy region depends not only on the masses of the three kinds of neutrinos, but also on the nuclear matrix element (or elements, since present and planned $0 \nu \beta \beta$ decay experiments [5-18] may consider about a dozen different nuclei) of a subtle two-nucleon operator between the ground states of the decaying nucleus and its decay product. Since $0 \nu \beta \beta$ decay involves not only nuclear physics but also unknown neutrino properties, such as the neutrino mass scale, the matrix elements cannot be measured; they must be calculated. And at present they are not calculated with much accuracy. We need to know them better.

Fortunately, nuclear-structure theory has made rapid progress in the last decade and the community is now in a position to improve calculated matrix elements materially. This review describes work that has already been carried out, from early pioneering studies to more recent and sophisticated efforts, and discusses what is needed to do significantly better. We are optimistic that recent progress in the use of chiral effective field theory $(\chi$ EFT $)$ to understand nuclear interactions [19-22], and of nonperturbative methods to efficiently solve the nuclear many-body problem from first principles (with controlled errors) [23-28] will produce reliable matrix elements with quantified uncertainties over the next five or so years. We will outline the ways in which that might happen.

This review is structured as follows: Section II discusses the significance of $0 \nu \beta \beta$ decay and the nuclear matrix elements that govern it. Section III reviews calculations of the matrix elements and indicates where we stand at present. Section IV is a slight detour into a more general problem, the "renormalization of the axial vector coupling $g_{A}$," that has important consequences for $0 \nu \beta \beta$ nuclear matrix elements. Section $\mathrm{V}$ is about ways in which matrix-element calculations should improve in the next few years, and ways in which the uncertainty in new calculations can be assessed. Section VI is a conclusion.

\section{SIGNIFICANCE OF DOUBLE-BETA DECAY}

\section{A. Neutrino Masses and Hierarchy}

Before turning to nuclear-structure theory, we very briefly review the neutrino physics that makes it necessary. References [29] and [30] contain pedagogical reviews of both the neutrino physics and the nuclear matrix elements that are relevant for $\beta \beta$ decay.

Flavor oscillations of neutrinos from the atmosphere [31], from the sun [32], and from nuclear reactors [33] have revealed neutrino properties that were unknown a few decades ago. Neutrinos have mass, but the three kinds of neutrino with well-defined masses are linear combinations of the kinds with definite flavor that interact in weak processes. We know with reasonable accuracy the differences in squared mass among the three

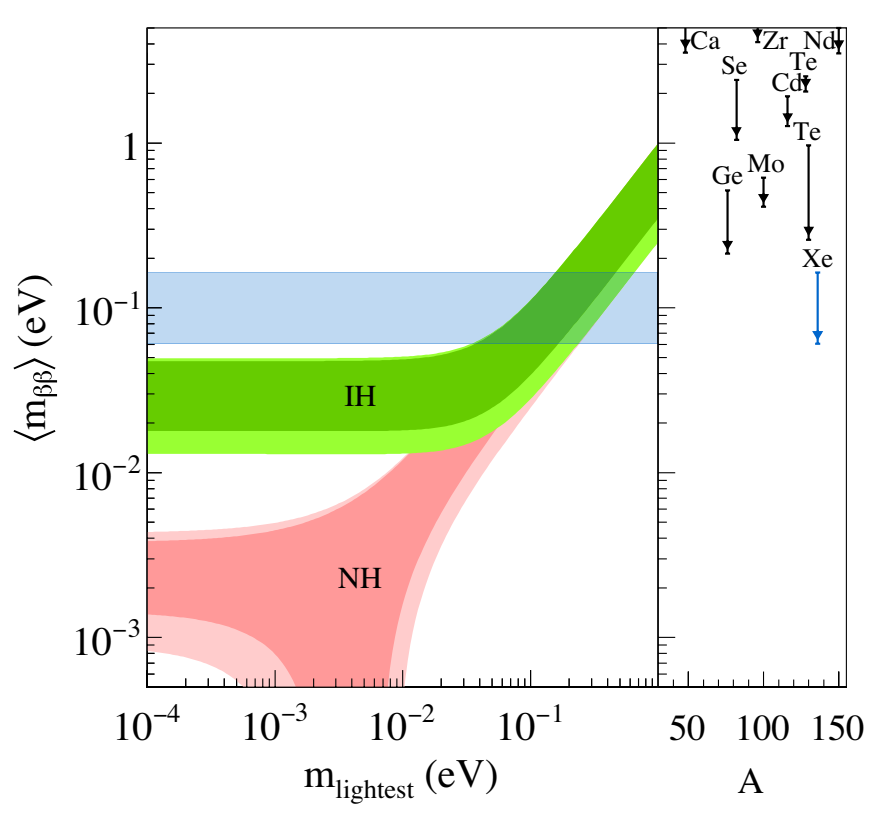

FIG. 1. Left panel: Bands for the value of the parameter $m_{\beta \beta}$ as a function of the mass of the lightest neutrino, for the case of normal (NH, red band) and inverted ( $\mathrm{IH}$, green band) neutrino-mass hierarchies. The present best experimental upper limits on $m_{\beta \beta}$ are shown in the blue band. Right panel: Present best upper limits, with uncertainty bars, on $m_{\beta \beta}$ from experiments performed on each $\beta \beta$ emitter, as a function of mass number $A$. The uncertainty bands and bars include experimental uncertainties and ranges of calculated nuclear matrix elements. Figure adapted from Ref. [5], courtesy of the KamLAND-Zen collaboration.

mass eigenstates, with one smaller difference $\Delta m_{\text {sun }}^{2} \simeq$ $75 \mathrm{meV}^{2}[34]$ coming mainly from solar-neutrino experiments and one larger difference $\Delta m_{\mathrm{atm}}^{2} \simeq 2400 \mathrm{meV}^{2}[34]$ coming mainly from atmospheric-neutrino experiments. We also know, with comparable accuracy, the mixing angles that specify which linear combinations of flavor eigenstates have definite mass [35].

The arrangement of the masses, called the "hierarchy," is still unknown, however. There are two possibilities: either the two mass eigenstates that mix most strongly with electron flavor are lighter than the third (the "normal hierarchy," because it is similar to the hierarchy of quark mass eigenstates) or they are heavier (the "inverted hierarchy"). Long baseline neutrino-oscillation experiments can eventually determine the hierarchy with a confidence level corresponding to four standard deviations or more, but for now they show just a two- $\sigma$ preference for the normal hierarchy $[36,37]$. Figure 1 shows the present experimental $0 \nu \beta \beta$ decay limits on the combination of neutrino masses $m_{\beta \beta}$ [defined by Eq. (5) in Sec. II B 1], together with the regions corresponding to the normal and inverted hierarchies, as a function of the mass of the lightest neutrino. If the hierarchy is normal and the lightest neutrino is lighter than about $10 \mathrm{meV}$, then a de- 
tection of $0 \nu \beta \beta$ decay is out of reach for the coming generation of experiments unless the decay is driven by the exchange of a heavy particle, the existence of which we have not yet discovered, or some other new physics (see Sec. II B 2). If the hierarchy is inverted, the experiments to take place in the next decade have a good chance to see the decay, provided they have enough material. Indeed, Fig. 1 shows that the current experimental limit almost touches the upper part of the inverted-hierarchy region.

How much material will be needed to completely cover the region, so that we can conclude in the absence of a $0 \nu \beta \beta$ signal that either the neutrino hierarchy is normal or neutrinos are Dirac particles? And in the event of a signal, how will we tell whether the exchange of light neutrinos or some other mechanism is responsible? If it is the latter, what is the underlying new physics? To answer any of these questions, we need accurate nuclear matrix elements.

\section{B. Neutrinoless Double-Beta Decay}

\section{Light-neutrino Exchange}

The beginning of this section closely follows Ref. [29], which itself is informed by Ref. [38]. More detailed derivations of the $\beta \beta$ transition rates can be found in Refs. [39-41].

The rate for $0 \nu \beta \beta$ decay, if we assume that it is mediated by the exchange of the three light Majorana neutrinos and the Standard Model weak interaction as represented in Fig. 2, is

$\left[T_{1 / 2}^{0 \nu}\right]^{-1}=\sum_{\text {spins }} \int\left|Z_{0 \nu}\right|^{2} \delta\left(E_{e 1}+E_{e 2}+E_{f}-E_{i}\right) \frac{d^{3} \boldsymbol{p}_{1}}{2 \pi^{3}} \frac{d^{3} \boldsymbol{p}_{2}}{2 \pi^{3}}$,

where $E_{e 1}, E_{e 2}$ and $\boldsymbol{p}_{1}, \boldsymbol{p}_{2}$ are the energies and momenta of the two emitted electrons, $E_{i}$ and $E_{f}$ are the energies of the initial and final nuclear states, and $Z_{0 \nu}$ is an amplitude proportional to an $S$-matrix element up to delta functions that enforce energy and momentum conservation. The $S$ matrix depends on the product of leptonic and hadronic currents in the effective low-energy semileptonic Lagrangian density:

$$
\mathcal{L}(x)=G_{F} / \sqrt{2}\left\{\bar{e}(x) \gamma_{\mu}\left(1-\gamma_{5}\right) \nu_{e}(x) J_{L}^{\mu}(x)\right\}+\text { h.c. },
$$

with $J_{L}^{\mu}$ the left-handed charge-changing hadronic current density. Because $Z_{0 \nu}$ is second order in the weakinteraction Lagrangian, it contains a lepton part that depends on two space-time positions $x$ and $y$, which are contracted and ultimately integrated over:

$$
\begin{aligned}
& \sum_{k} \bar{e}(x) \gamma_{\mu}\left(1-\gamma_{5}\right) U_{e k} \nu_{k}(x) \bar{e}(y) \gamma_{\nu}\left(1-\gamma_{5}\right) U_{e k} \nu_{k}(y) \\
= & -\sum_{k} \bar{e}(x) \gamma_{\mu}\left(1-\gamma_{5}\right) U_{e k} \nu_{k}(x) \overline{\nu_{k}^{c}}(y) \gamma_{\nu}\left(1+\gamma_{5}\right) U_{e k} e^{c}(y) .
\end{aligned}
$$

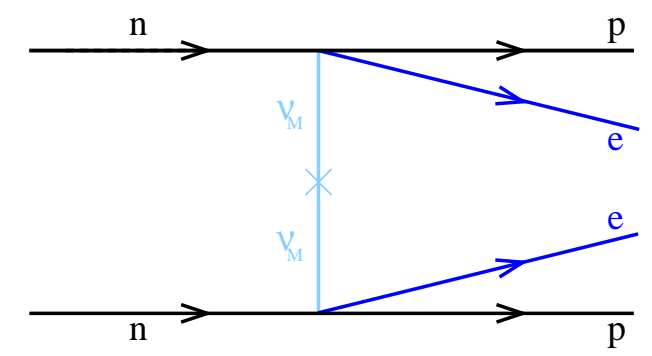

FIG. 2. Feynman diagram for $0 \nu \beta \beta$ decay mediated by lightneutrino exchange. Two neutrons (n) decay into two protons (p), emitting two electrons (e). No neutrinos are emitted, implying that they are Majorana particles $\left(\nu_{M}\right)$.

Here $\nu_{k}$ is the Majorana mass eigenstate with mass $m_{k}$ and $U_{e k}$ is the element of the neutrino mixing matrix that connects electron flavor with mass eigenstate $k$. We denote the charge conjugate of a field $\psi$ by $\psi^{c} \equiv i \gamma^{2} \psi^{*}$ (in the Pauli-Dirac representation), and because $\nu_{k}$ are Majorana states we can take $\nu_{k}^{c}=\nu_{k}$.

The contraction of $\nu_{k}$ with $\overline{\nu_{k}^{c}}$ turns out to be the usual fermion propagator, so that the lepton part above becomes

$$
\begin{aligned}
-\frac{i}{4} \int \sum_{k} \frac{d^{4} q}{(2 \pi)^{4}} e^{-i q \cdot(x-y)} \bar{u}\left(p_{1}\right) \gamma_{\mu}\left(1-\gamma_{5}\right) e^{-i\left(p_{1} \cdot x+p_{2} \cdot y\right)} \\
\times \frac{q+m_{k}}{q^{2}-m_{k}^{2}} \gamma_{\nu}\left(1+\gamma_{5}\right) u^{c}\left(p_{2}\right) U_{e k}^{2}
\end{aligned}
$$

where $q$ is the 4-momentum of the virtual neutrino. The term with $q$ vanishes because the two currents are left handed and if we neglect the very small neutrino masses in the denominator, the decay amplitude becomes proportional to

$$
\begin{aligned}
& m_{\beta \beta} \equiv\left|\sum_{k} m_{k} U_{e k}^{2}\right| \\
& =\left.\left|m_{1}\right| U_{e 1}\right|^{2}+m_{2}\left|U_{e 2}\right|^{2} e^{i\left(\alpha_{2}-\alpha_{1}\right)}+m_{3}\left|U_{e 3}\right|^{2} e^{i\left(-\alpha_{1}-2 \delta\right)} \mid .
\end{aligned}
$$

Here $\delta$ is the so-called Dirac phase, and $\alpha_{1}, \alpha_{2}$ are Majorana phases that vanish if neutrinos are Dirac particles. We have inserted the absolute value in Eq. (5) consistently with the amplitude in Eq. (1), because the expression inside can be complex.

To obtain the full amplitude $Z_{0 \nu}$, one must multiply the lepton part above by the nuclear matrix element of two time-ordered hadronic currents and integrate the product over $x$ and $y$. Because $J_{L}^{\mu}(x)=$ $e^{i H x_{0}} J_{L}^{\mu}(\boldsymbol{x}) e^{-i H x_{0}}$ ( $H$ is the hadronic Hamiltonian and the current on the right-hand side is evaluated at time $x_{0}=0$ ), one can write the matrix element of an ordinary product of hadronic currents between initial (i) and final 
(f) nuclear states as

$$
\begin{aligned}
\left\langle f\left|J_{L}^{\mu}(x) J_{L}^{\nu}(y)\right| i\right\rangle=\sum_{n} & \left\langle f\left|J_{L}^{\mu}(\boldsymbol{x})\right| n\right\rangle\left\langle n\left|J_{L}^{\nu}(\boldsymbol{y})\right| i\right\rangle \\
& \times e^{-i\left(E_{n}-E_{f}\right) x_{0}} e^{-i\left(E_{i}-E_{n}\right) y_{0}},
\end{aligned}
$$

where the $|n\rangle$ 's are a complete set of intermediate nuclear states, with corresponding energies $E_{n}$. Time ordering the product of currents and combining the phases in Eq. (6) with similar factors from the lepton currents yields the following amplitude, after integration first over $x_{0}, y_{0}$, and $q^{0}$, then over $\boldsymbol{x}$ and $\boldsymbol{y}$ :

$$
\begin{aligned}
\sum_{n} & {\left[\frac{\left\langle f\left|J_{L}^{\mu}(\boldsymbol{q})\right| n\right\rangle\left\langle n\left|J_{L}^{\nu}(-\boldsymbol{q})\right| i\right\rangle}{|\boldsymbol{q}|\left(E_{n}+|\boldsymbol{q}|+E_{e 2}-E_{i}\right)}+\frac{\left\langle f\left|J_{L}^{\nu}(\boldsymbol{q})\right| n\right\rangle\left\langle n\left|J_{L}^{\mu}(-\boldsymbol{q})\right| i\right\rangle}{|\boldsymbol{q}|\left(E_{n}+|\boldsymbol{q}|+E_{e 1}-E_{i}\right)}\right] } \\
& \times 2 \pi \delta\left(E_{f}+E_{e 1}+E_{e 2}-E_{i}\right)
\end{aligned}
$$

where the tiny neutrino masses in the denominator of Eq. (4) and the electron momenta $\left|\boldsymbol{p}_{1}\right|$ and $\left|\boldsymbol{p}_{2}\right|$ have been neglected because they are much smaller than a typical momentum transfer $|\boldsymbol{q}|$. The energy-conservation condition comes from the definition of $Z_{0 \nu}$.

To go further one needs to know the nuclear current operators. At this point, most authors make two important approximations. The first is the "impulse approximation," i.e. the use of the current operator for a collection of free nucleons. The operator is then specified by its one-body matrix elements:

$$
\begin{aligned}
\left\langle p\left|J_{L}^{\mu}(x)\right| p^{\prime}\right\rangle= & e^{i q x} \bar{u}(p)\left(g_{V}\left(q^{2}\right) \gamma^{\mu}-g_{A}\left(q^{2}\right) \gamma_{5} \gamma^{\mu}\right. \\
& \left.-i g_{M}\left(q^{2}\right) \frac{\sigma^{\mu \nu}}{2 m_{N}} q_{\nu}+g_{P}\left(q^{2}\right) \gamma_{5} q^{\mu}\right) u\left(p^{\prime}\right)
\end{aligned}
$$

where $q=p^{\prime}-p$, the conservation of the vector current tells us that $g_{V} \equiv g_{V}(0)=1$, and $g_{M}\left(q^{2}\right)=g_{M} g_{V}\left(q^{2}\right)$ with $g_{M} \equiv g_{M}(0) \simeq 4.70 g_{V}$ (as given by the proton and neutron anomalous magnetic moments [34]), $g_{A}=g_{A}(0) \simeq 1.27$ [34] (from neutron $\beta$-decay measurements [42]), and the Goldberger-Treiman relation $g_{P}\left(q^{2}\right)=2 m_{N} g_{A}\left(q^{2}\right) /\left(\boldsymbol{q}^{2}+m_{\pi}^{2}\right)$, with $m_{N}$ and $m_{\pi}$ the nucleon and pion masses, connects the pseudoscalar and axial terms and is accurate enough for our purposes. The momentum-transfer dependence of the axial and vector terms can be parameterized in several ways by fitting experimental data [43, 44]. A non-relativistic reduction of the matrix elements in Eq. (8) leads to the form $J_{L}(\boldsymbol{q})=\sum_{a} \exp \left(-i \boldsymbol{q} \cdot \boldsymbol{x}_{a}\right) \hat{O}\left(\boldsymbol{x}_{a}\right) \tau_{a}^{+}$, where the operator $\hat{O}\left(\boldsymbol{x}_{a}\right)$ acts on space and spin variables of the $a^{\text {th }}$ nucleon and the isospin-raising $\tau_{a}^{+}$operator makes the nucleon a proton if it is initially a neutron.

The second approximation, known as closure, begins with the observation that to contribute significantly to the amplitude, the momentum transfer must be on the order of an average inverse spacing between nucleons, about $100 \mathrm{MeV}$. The closure approximation is to neglect the intermediate-state-dependent quantity $E_{n}-E_{i}$ (which is generally small compared to $|\boldsymbol{q}|$ ) in the denominator of Eq. (7), so that $E_{n}$ can be replaced by a state-independent average value $\bar{E}$ and the contributions of intermediate states can be summed implicitly in Eq. (7). This approximation avoids the explicit calculation of excited states of the intermediate odd-odd nucleus up to high energies, a nuclear structure calculation that is computationally much more involved than obtaining the initial and final states in the decay. Because the momentum transfer in $2 \nu \beta \beta$ decay (limited by the $\mathrm{Q}$-value of the transition) is of the same order of magnitude as $E_{n}-E_{i}$, the closure approximation cannot be used there. For that reason, some methods that focus on low-lying states or even-even nuclei can be applied to $0 \nu \beta \beta$ decay but not to $2 \nu \beta \beta$ decay. Approaches that do allow an evaluation of the contributions of each intermediate state suggest that a sensible choice of $\bar{E}$ can allow the closure approximation to reproduce the unapproximated $0 \nu \beta \beta$ matrix element to within $10 \%$ [45-49]. It is worth noting, however, that tests of the closure approximation have not included states above 10's of MeV. Since higher-energy/shorterrange dynamics could be important, future closure tests should include them.

Assuming the closure approximation is accurate, and neglecting terms associated with the emission of $p$-wave electrons (which are expected to be a few percent of those associated with $s$-wave electrons) and the small electron energies $\left(E_{e 1}-E_{e 2}\right) / 2$ in the denominator of Eq. (7), one has the expression

$$
\left[T_{1 / 2}^{0 \nu}\right]^{-1}=G_{0 \nu}(Q, Z)\left|M_{0 \nu}\right|^{2} m_{\beta \beta}^{2},
$$

where $Q \equiv E_{i}-E_{f}, Z$ is the proton number, and $G_{0 \nu}(Q, Z)$ comes from the phase-space integral and has recently been re-evaluated with improved precision $[50,51]$. The "nuclear matrix element" $M_{0 \nu}[52-54]$ is given by

$$
M_{0 \nu}=M_{0 \nu}^{G T}-\frac{g_{V}^{2}}{g_{A}^{2}} M_{0 \nu}^{F}+M_{0 \nu}^{T},
$$

with, in the approximations mentioned above, 


$$
\begin{aligned}
M_{0 \nu}^{G T} & =\frac{2 R}{\pi g_{A}^{2}} \int_{0}^{\infty}|\boldsymbol{q}| d|\boldsymbol{q}|\left\langle f\left|\sum_{a, b} \frac{j_{0}\left(|\boldsymbol{q}| r_{a b}\right) h_{G T}(|\boldsymbol{q}|) \boldsymbol{\sigma}_{a} \cdot \boldsymbol{\sigma}_{b}}{|\boldsymbol{q}|+\bar{E}-\left(E_{i}+E_{f}\right) / 2} \tau_{a}^{+} \tau_{b}^{+}\right| i\right\rangle, \\
M_{0 \nu}^{F} & =\frac{2 R}{\pi g_{A}^{2}} \int_{0}^{\infty}|\boldsymbol{q}| d|\boldsymbol{q}|\left\langle f\left|\sum_{a, b} \frac{j_{0}\left(|\boldsymbol{q}| r_{a b}\right) h_{F}(|\boldsymbol{q}|)}{|\boldsymbol{q}|+\bar{E}-\left(E_{i}+E_{f}\right) / 2} \tau_{a}^{+} \tau_{b}^{+}\right| i\right\rangle, \\
M_{0 \nu}^{T} & =\frac{2 R}{\pi g_{A}^{2}} \int_{0}^{\infty}|\boldsymbol{q}| d|\boldsymbol{q}|\left\langle f\left|\sum_{a, b} \frac{j_{2}\left(|\boldsymbol{q}| r_{a b}\right) h_{T}(|\boldsymbol{q}|)\left[3 \boldsymbol{\sigma}_{j} \cdot \hat{\boldsymbol{r}}_{a b} \boldsymbol{\sigma}_{k} \cdot \hat{\boldsymbol{r}}_{a b}-\boldsymbol{\sigma}_{a} \cdot \boldsymbol{\sigma}_{b}\right]}{|\boldsymbol{q}|+\bar{E}-\left(E_{i}+E_{f}\right) / 2} \tau_{a}^{+} \tau_{b}^{+}\right| i\right\rangle .
\end{aligned}
$$

Here the nucleon coordinates are all operators that, like spin and isospin operators, act on nuclear states. The nuclear radius, $R$, is inserted by convention to make the matrix element dimensionless, with a compensating factor in $G_{0 \nu}$ in Eq. (9). The quantity $r_{a b}=\left|\boldsymbol{x}_{a}-\boldsymbol{x}_{b}\right|$ is the magnitude of the inter-nucleon position vector, and $\hat{r}_{a b}=\left(\boldsymbol{x}_{a}-\boldsymbol{x}_{b}\right) / r_{a b}$ is the corresponding unit vector. The objects $j_{0}$ and $j_{2}$ denote spherical Bessel functions, and the $h$ 's, called neutrino potentials, are defined in momentum space by

$$
\begin{gathered}
h_{G T}(|\boldsymbol{q}|) \equiv g_{A}^{2}\left(\boldsymbol{q}^{2}\right)-\frac{g_{A}\left(\boldsymbol{q}^{2}\right) g_{P}\left(\boldsymbol{q}^{2}\right) \boldsymbol{q}^{2}}{3 m_{N}} \\
\quad+\frac{g_{P}^{2}\left(\boldsymbol{q}^{2}\right) \boldsymbol{q}^{4}}{12 m_{N}^{2}}+\frac{g_{M}^{2}\left(\boldsymbol{q}^{2}\right) \boldsymbol{q}^{2}}{6 m_{N}^{2}}, \\
h_{F}(|\boldsymbol{q}|) \equiv \frac{g_{V}^{2}\left(\boldsymbol{q}^{2}\right)}{g_{A}^{2}}, \\
h_{T}(|\boldsymbol{q}|) \equiv \frac{g_{A}\left(\boldsymbol{q}^{2}\right) g_{P}\left(\boldsymbol{q}^{2}\right) \boldsymbol{q}^{2}}{3 m_{N}}-\frac{g_{P}^{2}\left(\boldsymbol{q}^{2}\right) \boldsymbol{q}^{4}}{12 m_{N}^{2}}+\frac{g_{M}^{2}\left(\boldsymbol{q}^{2}\right) \boldsymbol{q}^{2}}{12 m_{N}^{2}},
\end{gathered}
$$

where terms of higher order in $1 / m_{N}$, coming from the nonrelativistic expansion of Eq. (8), have been neglected.

Sometimes the operators inside the matrix elements of Eq. (11) are multiplied by a radial function $f\left(r_{a b}\right)$, designed to take into account short-range correlations that are omitted by Hilbert-space truncation in most manybody calculations. Several parameterizations of $f$ have been proposed; they are based either based on a Jastrow ansatz [55], the unitary correlator operator method [56], Brueckner-Goldstone calculations $[57,58]$ or nuclear matter correlation functions [59]. Even though the prescriptions differ from one another, those that preserve isospin symmetry (the Jastrow ansatz does not [60]) have small effects on $0 \nu \beta \beta$ matrix elements when the momentum dependence of the transition operator is taken fully into account $[61,62]$.

For completeness, we write down the decay rate for $2 \nu \beta \beta$ decay, which is permitted in the Standard Model and therefore does not depend on neutrino mass or charge-conjugation properties. This process is sketched in Fig. 3 and the decay rate can be derived in a similar way as for $0 \nu \beta \beta$ decay, with the result that

$$
\left[T_{1 / 2}^{2 \nu}\right]^{-1}=G_{2 \nu}(Q, Z)\left|M_{G T}^{2 \nu}-\frac{g_{V}^{2}}{g_{A}^{2}} M_{F}^{2 \nu}\right|^{2},
$$

where $G_{2 \nu}(Q, Z)$ is the corresponding phase-space factor

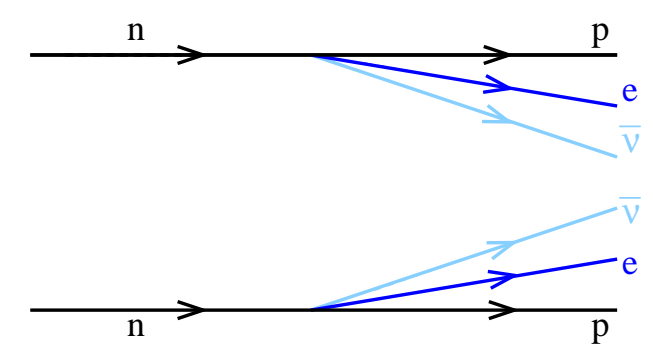

FIG. 3. Feynman diagram for $2 \nu \beta \beta$ decay.

(also calculated to high precision in Refs. [50, 51]) and

$$
\begin{aligned}
M_{F}^{2 \nu} & =\sum_{n} \frac{\left\langle f\left|\sum_{a} \tau_{a}^{+}\right| n\right\rangle\left\langle n\left|\sum_{b} \tau_{b}^{+}\right| i\right\rangle}{E_{n}-\left(M_{i}+M_{f}\right) / 2}, \\
M_{G T}^{2 \nu} & =\sum_{n} \frac{\left\langle f\left|\sum_{a} \boldsymbol{\sigma}_{a} \tau_{a}^{+}\right| n\right\rangle\left\langle n\left|\sum_{b} \boldsymbol{\sigma}_{b} \tau_{b}^{+}\right| i\right\rangle}{E_{n}-\left(M_{i}+M_{f}\right) / 2} .
\end{aligned}
$$

Isospin symmetry forces all the Fermi strength to lie in the isobar analog state in the daughter nucleus, so that $M_{F}^{2 \nu}$ for the transition to the daughter ground state is negligibly small.

\section{New Physics Mechanisms}

It is not just light-neutrino exchange that can contribute to $0 \nu \beta \beta$ decay. Although the occurrence of $0 \nu \beta \beta$ decay immediately implies that neutrinos are Majorana particles [63] — once a lepton-number violating operator appears in the Lagrangian, all possible effective operators violating the symmetry are generated - some other lepton-number violating mechanism could be the dominant cause of the decay $[64,65]$. If that were the case, the detection of $0 \nu \beta \beta$ decay would not give us information about the absolute neutrino mass, but could be used as a low-energy test of new physics that would complement high-energy searches at accelerators such as the Large Hadron Collider [66-69].

Several mechanisms for $0 \nu \beta \beta$ decay have been proposed. Besides the exchange of sterile neutrinos via left-handed currents [70], the most popular are the exchange of light or heavy neutrinos in left-right symmetric models [71, 72], the exchange of supersymmetric particles [73, 74], and the emission of Majorons (bosons 
that appear in theories with spontaneous breaking of the global baryon-lepton number symmetry $B-L$ [75-77]). Combining the contributions from all possible mechanisms, one finds that the $0 \nu \beta \beta$ decay rate takes the form

$$
\left[T_{1 / 2}^{0 \nu}\right]^{-1}=\sum_{i} G_{0 \nu}^{i}(Q, Z)\left|M_{i}^{0 \nu}\right|^{2} \eta_{i}^{2}
$$

with new-physics parameters $\eta_{i}$ that are distinct for every mechanism and mode: a combination of the light neutrino masses $\left(m_{\beta \beta}\right)$ for the usual mechanism and combinations of heavy-neutrino masses, the right-handed $W_{R}$ boson mass, the left- and right-handed $W_{L}-W_{R}$ boson mixing angles, supersymmetric couplings, couplings of Majorons to neutrinos, etc. for nonstandard mechanisms. It is these parameters that $0 \nu \beta \beta$ decay experiments can constrain, provided that the associated nuclear matrix elements $M_{i}^{0 \nu}$ are known. (Updated phase-space factor calculations can be found in Ref. [78].) Detailed treatments of the matrix elements governing these newphysics $0 \nu \beta \beta$ decay modes appear in Refs. [30, 39-41]. Matrix elements calculations for the sterile-neutrino exchange [70, 79-82], left-right symmetric models [78, 8385], and the exchange of supersymmetric particles [86-89] are common in the literature.

Most of the new-physics mechanisms involve the exchange of heavy particles. However, the direct exchange between nucleons, represented by the contact operator in the bottom diagram in Fig. 4 in the heavy-particle limit, occurs less often in most models than exchange between pions or between a pion and a nucleon, shown in the top and middle diagrams of the figure. In $\chi \mathrm{EFT}$ each pion propagator carries a factor $\Lambda_{b}^{2} / m_{\pi}^{2}$, where $\Lambda_{b} \sim 500$ $\mathrm{MeV}-1 \mathrm{GeV}$ is the chiral-symmetry breaking scale, at which the effective theory breaks down. Each ordinary two-nucleon-pion $(N N \pi)$ vertex comes with a derivative, which results in a factor of $p / \Lambda_{b}$ or $m_{\pi} / \Lambda_{b}$, where $p$ is a typical momentum. Because the contact interaction has no derivatives in most models, pion mediation enhances the amplitude [90]. The two-pion mode at the top of the figure is thus generally the dominant one. The onepion graph in the middle is nominally smaller by a factor of $\Lambda_{b} / m_{\pi}$ and the four-nucleon graph at the bottom is smaller by another factor of the same quantity. The leading one-pion-exchange contribution to $0^{+} \rightarrow 0^{+} 0 \nu \beta \beta$ decay is forbidden by parity symmetry, however, and so the middle graph ends up contributing at the same order as the contact term [90]. The counting is different for nuclear forces, where the contact and one-pion exchange interactions both appear at leading order $[19,20]$. The usual one-pion exchange interaction diagram contains a derivative at each vertex; the derivatives counteract the pion propagator, placing the diagram at the same chiral order as the four-nucleon contact diagram. Two-pion exchange occurs at higher order. Computations of matrix elements in supersymmetric models, even when they do not rely explicitly on $\chi \mathrm{EFT}$, support the statement that pion-exchange modes are the most important [91-93].

The $\chi$ EFT counting should be confirmed by explicit

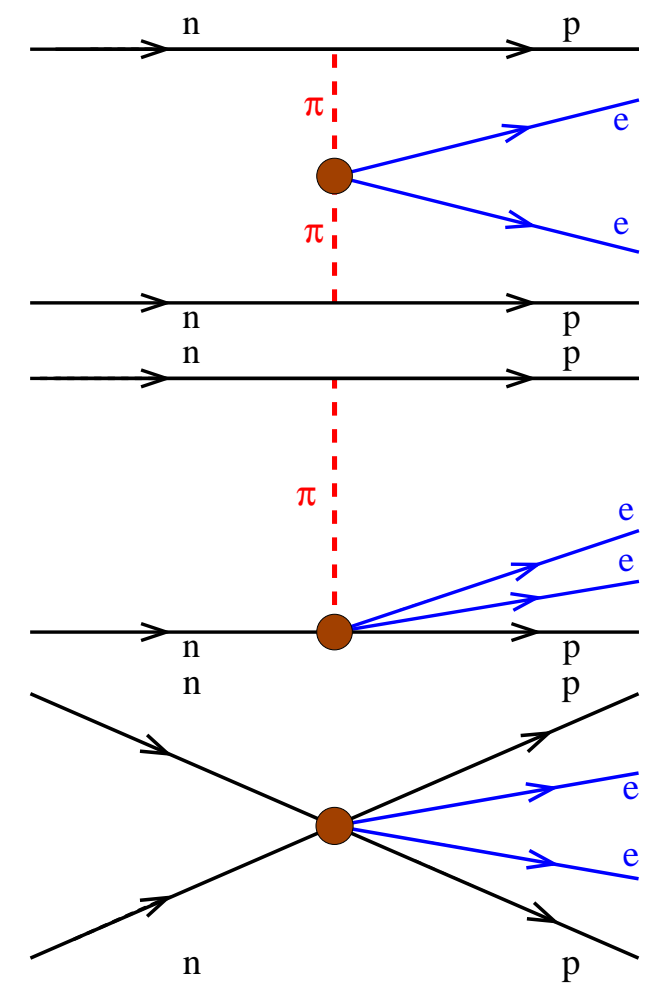

FIG. 4. Diagrams for the two-pion-exchange (top), one-pionexchange (middle) and contact (bottom) modes of $0 \nu \beta \beta$ decay caused by lepton-number violation associated with the exchange of a heavy particle.

calculations, as additional suppression or enhancement may occur [94]. Lattice QCD studies that explicitly incorporate hadronic degrees of freedom are underway [95], and will provide accurate input for the effective field theory treatment of these decay modes.

The four-nucleon contact vertex represented at the bottom of Fig. 4 is further affected by short-range physics. In the light-neutrino exchange $0 \nu \beta \beta$ decay mode, typical internucleon distances are of the order of few femtometers. The exchange of heavy particles, with mass $m_{H} \gtrsim 100 \mathrm{GeV}$ [90], requires nucleons to be closer to each other and will thus be suppressed. Pions have a mass of $m_{\pi} \simeq 138 \mathrm{MeV} \approx 1.4 \mathrm{fm}^{-1}$, a distance comparable to the average internucleon spacing, and so the graphs with pions propagating between nucleons will not be suppressed. This behavior is apparent in potentials associated with the three modes of heavy-particle exchange. In momentum space, they have the form

$$
\begin{aligned}
h_{N N-G T / N N-T}(|\boldsymbol{q}|) & \sim \boldsymbol{q}^{2}, \\
h_{\pi-G T / \pi-T}(|\boldsymbol{q}|) & \sim \frac{\boldsymbol{q}^{2}}{\boldsymbol{q}^{2}+m_{\pi}^{2}}, \\
h_{\pi \pi-G T / \pi \pi-T}(|\boldsymbol{q}|) & \sim \frac{\boldsymbol{q}^{2}}{\left(\boldsymbol{q}^{2}+m_{\pi}^{2}\right)^{2}} .
\end{aligned}
$$

The first of these is clearly more strongly affected at 
high momentum transfer than the two pion-exchange modes $^{1}$. Additional powers of the momentum transfer can be present in subleading contributions to each diagram.

The effects of short-range physics must be treated with care when working in $\chi \mathrm{EFT}$, from which that physics has effectively been integrated out, or in many-body approaches with severe truncation. The contact coupling constant must then be renormalized from its naive value by an amount that can be computed with the similarity renormalization group (SRG) [96, 97] or related schemes. Until such methods are perfected (we discuss progress in Sec. V) the strength of the contact term will carry some uncertainty. No such uncertainty plagues the strong contact interaction in the $\chi$ EFT Hamiltonian, the parameters of which are fit directly to data. It is fortunate that the $\beta \beta$ contact operators are less important than those involving pion exchange.

Within specific models, heavy-particle exchange with $m_{H}$ of the order of one $\mathrm{TeV}$ can compete with the lightneutrino exchange $[98,99]$. Once $0 \nu \beta \beta$ decay has been observed, therefore, one must cope with the question of its cause. The easiest process to distinguish from lightneutrino exchange is decay with the emission of a Majoron; the additional emitted particle causes the energy spectrum of the electron pairs to be spread out rather than concentrated at a single energy. More challenging for experiments is exploiting the angular correlation between the two electrons, which is different if one of them is emitted in a $p$-wave. The emission in $p$-waves is suppressed in light-neutrino exchange (where $s$-waves are always more likely), but turns out to be important in models with right-handed lepton currents [39, 85], where the parity-odd $q^{\rho} \gamma_{\rho}$ term in Eq. (4) contributes to the amplitude instead of the term containing the neutrino masses. Some upcoming $0 \nu \beta \beta$ decay experiments should be able to measure angular distributions, given enough events $[7,100]$.

Unfortunately, other kinds of new physics can produce the same angular electron correlations as does light neutrino exchange [101]. In those cases, however, one might be able to determine the physics responsible for the decay by combining measurements in different isotopes (such measurements will be required in any event to confirm detection). The matrix elements governing heavy-particlemediated and light-neutrino-mediated decay can depend differently on the nuclear species in which the decay occurs [85, 89, 102-104]. The task will be difficult, however, if the dependence is similar, as Refs. [105, 106] suggest. Another possibility is to compare the decay rates to the ground state and the first excited $0^{+}$state of the daughter nucleus; the ratio of these quantities can

\footnotetext{
1 The induced pseudoscalar term discussed in Sec. II B 1 also involves pion-exchange, in combination with the usual exchange of a light neutrino. There the pion brings no enhancement because the light-neutrino exchange is already long range.
}

also depend on the decay mode [107]. Unfortunately, it is difficult to observe the decay to an excited state, which is suppressed by the small $Q$-value associated with the transition [50]. The suppression is too great to be compensated for by differences in nuclear matrix elements [82, 107-110].

\section{Importance of Nuclear Matrix Elements for Experiments}

The next generation of $0 \nu \beta \beta$ decay experiments will aim to fully cover the region $m_{\beta \beta} \gtrsim 10 \mathrm{meV}$ in Fig. 1, so that a signal will be detected if neutrinos are Majorana particles and the mass hierarchy is inverted. As Eq. (9) shows, the $0 \nu \beta \beta$ decay lifetime depends on the square of the nuclear matrix element that we need to calculate. This sensitivity makes matrix elements calculations important in a number of ways.

First, if an experiment is completely background free, the amount of material needed to be sensitive to any particular neutrino mass $m_{\beta \beta}$ in a given time is proportional to the lifetime and thus, from Eq. (9), the inverse square of the matrix element. An uncertainty of a factor of three in the matrix element thus corresponds to nearly an order of magnitude uncertainty in the amount of material required, e.g., to cover the parameter space corresponding to the inverted hierarchy. If the experiment is background-limited, the uncertainty is even larger [111]. An informed decision about how much material to use in an expensive experiment will require a more accurate matrix element.

Second, the uncertainty affects the choice of material to be used in $0 \nu \beta \beta$ decay searches, a choice that is a compromise between experimental advantages and the matrix element value. Figure 5 (top) shows nuclear matrix elements calculated in different approaches, and because of the spread of the results (roughly the factor of three above) we can conclude only that the matrix element of ${ }^{48} \mathrm{Ca}$ is smaller than those of the other $0 \nu \beta \beta$ decay candidates. And the differences in the expected rate, a product of the nuclear matrix elements and phase-space factors, are even more similar (see Fig. 5 bottom, and Eq. (9)) [112]. Better calculations would make it easier to select an optimal isotope.

Finally, and perhaps most obviously, we need matrix elements to obtain information about the absolute neutrino masses once a $0 \nu \beta \beta$ decay lifetime is known. Reducing the uncertainty in the matrix element calculations will be crucial if we wish to fully exploit an eventual measurement of the decay half-life. Even the interpretation of limits is hindered by matrix-element uncertainty. The blue band in Figure 1 represents the upper limit of $m_{\beta \beta}<61-165 \mathrm{meV}$ from the KamLAND-Zen experiment [5]. The uncertainty, again a factor of about three, is due almost entirely to the matrix element. And the real theoretical uncertainty, at this point, must be taken to be larger; the " $g_{A}$ problem," which we discuss in Sec. IV, 
has been ignored in this analysis. We really need better calculations. Fortunately, we are now finally in a position to undertake them.

\section{NUCLEAR MATRIX ELEMENTS AT PRESENT}

As we have noted, calculated matrix elements at present carry large uncertainties. Matrix elements obtained with different nuclear-structure approaches differ by factors of two or three. Figure 5 compares matrix elements produced by the shell model [82, 113, 114], different variants of the quasiparticle random phase approximation (QRPA) [81, 115-117], the interacting boson model (IBM) [109], and energy density functional (EDF) theory [118-120]. The strengths and weaknesses of each calculation are discussed in detail later in this Section.

Some of these methods can be used to compute single$\beta$ and $2 \nu \beta \beta$ decay lifetimes. It is disconcerting to find that predicted lifetimes for these processes are almost always shorter than measured lifetimes, i.e. computed single Gamow-Teller and $2 \nu \beta \beta$ matrix elements are too large [121-123]. The problems are usually "cured" by reducing the strength of the spin-isospin Gamow-Teller operator $\boldsymbol{\sigma} \boldsymbol{\tau}$, which is equivalent to using an effective value of the axial coupling constant that multiplies this operator in place of its "bare" value of $g_{A} \simeq 1.27$. This phenomenological modification is sometimes referred to as the "quenching" or "renormalization" of $g_{A}$. In Sec. IV we review possible sources of the renormalization, none of which has yet been shown to fully explain the effect, and their consequences for $0 \nu \beta \beta$ matrix elements.

\section{A. Shell Model}

The nuclear shell model is a well-established manybody method, routinely used to describe the properties of medium-mass and heavy nuclei [121, 124, 125], including candidates for $\beta \beta$-decay experiments. The model, also called the "configuration interaction method" (particularly in quantum chemistry $[126,127]$ ), is based on the idea that the nucleons near the Fermi level are the most important for low-energy nuclear properties, and that all the correlations between these nucleons are relevant. Thus, instead of solving the Schrödinger equation for the full nuclear interaction in the complete manybody Hilbert space, one restricts the dynamics to a limited configuration space (sometimes called the valence space) containing only a subset of the system's nucleons. In the configuration space one uses an effective nuclear interaction $H_{\text {eff }}$, defined (ideally) so that the observables of the full-space calculation are reproduced, e.g.

$$
H\left|\Phi_{i}\right\rangle=E_{i}\left|\Phi_{i}\right\rangle \rightarrow H_{\mathrm{eff}}\left|\bar{\Phi}_{i}\right\rangle=E_{i}\left|\bar{\Phi}_{i}\right\rangle .
$$

The states $\left|\Phi_{i}\right\rangle$ and $\left|\bar{\Phi}_{i}\right\rangle$ are defined in the full space and the configuration space, respectively, and have associated

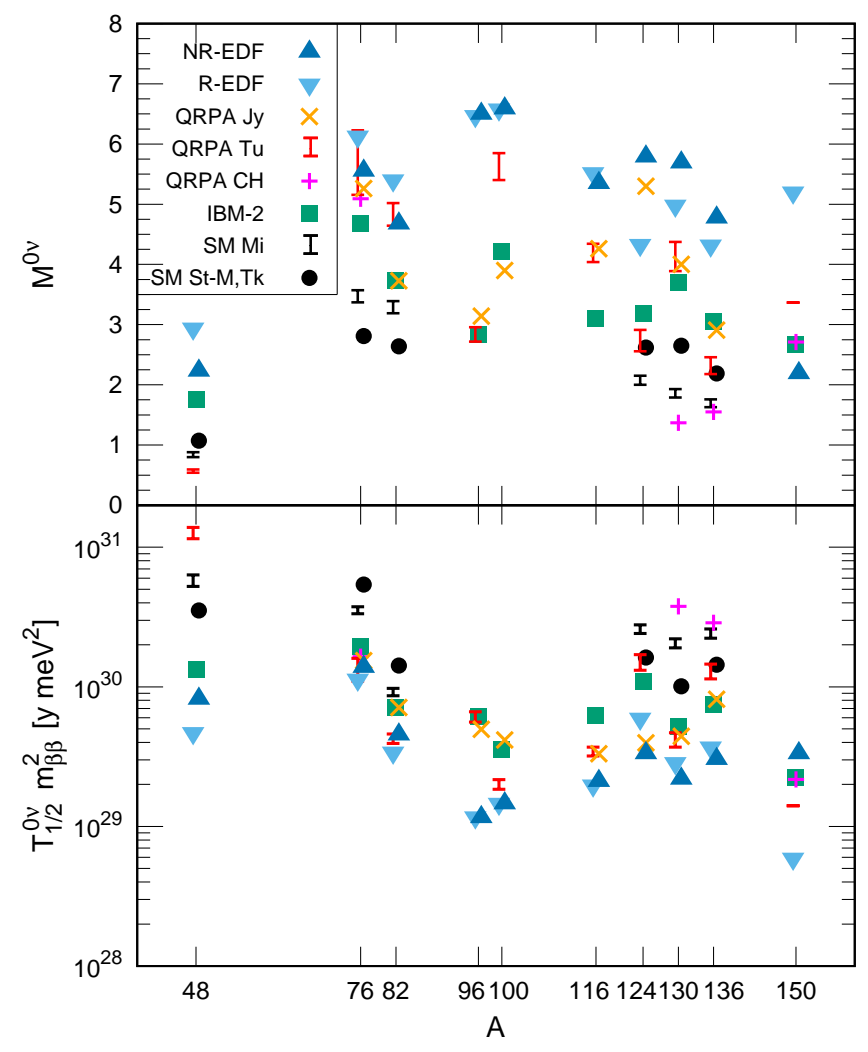

FIG. 5. Top panel: Nuclear matrix elements $\left(M^{0 \nu}\right)$ for $0 \nu \beta \beta$ decay candidates as a function of mass number $A$. All the plotted results are obtained with the assumption that the axial coupling constant $g_{A}$ is unquenched and are from different nuclear models: the shell model (SM) from the StrasbourgMadrid (black circles) [113], Tokyo (black circle in ${ }^{48} \mathrm{Ca}$ ) [114], and Michigan (black bars) [82] groups; the interacting boson model (IBM-2, green squares) [109]; different versions of the quasiparticle random-phase approximation (QRPA) from the Tübingen (red bars) [115, 116], Jyväskylä (orange times signs) [81], and Chapel Hill (magenta crosses) [117] groups; and energy density functional theory (EDF), relativistic (downside cyan triangles) $[118,119]$ and non-relativistic (blue triangles) [120]. QRPA error bars result from the use of two realistic nuclear interactions, while shell model error bars result from the use of several different treatments of short range correlations. Bottom panel: Associated $0 \nu \beta \beta$ decay half-lives, scaled by the square of the unknown parameter $m_{\beta \beta}$.

energy $E_{i}$.

The configuration space usually comprises only a relatively small number of "active" nucleons outside a core of nucleons that are frozen in the lowest-energy orbitals and not included in the calculation. The active nucleons can occupy only a limited set of single-particle levels around the Fermi surface. Many-body states are linear combinations of orthogonal Slater determinants $\left|\psi_{i}\right\rangle$ (usually from a harmonic-oscillator basis) for nucleons in those 
single-particle states,

$$
\left|\bar{\Phi}_{i}\right\rangle=\sum_{j} c_{i j}\left|\psi_{j}\right\rangle
$$

with the $c_{i j}$ determined by exact diagonalization of $H_{\text {eff }}$.

The shell model describes ground-state nuclear properties such as masses, separation energies, and charge radii quite well. It also does a good job with low-lying excitation spectra and with electric moments and transitions [121, 124, 125] if appropriate effective charges are used [128]. The wide variety of successes over a broad range of isotopes reflects the shell model's ability to capture both the excitation of a single particle from an orbital below the Fermi surface to one above, in the spirit of the original naive shell model [129, 130], and collective correlations that come from the coherent motion of many nucleons in the configuration space. The exact diagonalization of $H_{\text {eff }}$ means that the shell model states $\left|\bar{\Phi}_{i}\right\rangle$ contain all correlations (isovector and isoscalar pairing, quadrupole collectivity, etc.) that can be induced by $H_{\text {eff. }}$.

This careful treatment of correlations, on the other hand, restricts the range of shell model to relatively small configuration spaces, at present those for which the Hilbert-space dimension is less than about $\left(10^{11}\right)[131$, 132]. For this reason most shell model calculations of $0 \nu \beta \beta$ decay have been performed in a single harmonicoscillator shell, consisting of four or five single-particle orbitals, not counting degeneracies from rotational invariance, for both protons and neutrons $[47-49,54,82$, 108, 113, 133-135]. Enlarging the configuration space increases the number of active nucleons, leading to a Hilbert-space dimension that increases combinatiorally with the number of active-nucleon configurations in the valence space and quickly making dimensions intractable. (Very recently, however, the authors of Ref. [114] performed the first calculation in two oscillator shells; we discuss it in detail in Sec. IIIF.)

Pairing correlations, which are central in nuclear structure and have large effects on $0 \nu \beta \beta$ decay, may not be fully captured by $H_{\text {eff }}$ within a single oscillator shell [136]. In addition, because the one-body spin-orbit interaction significantly lowers the energy of orbitals with spin parallel to the orbital angular momentum in heavy nuclei, spin-orbit partners are split by several $\mathrm{MeV}$ or more, and the shell-model configuration space contains only one member of some spin-orbit pair (except in ${ }^{48} \mathrm{Ca}$ ). The omission may have important consequences because the spin-isospin part of the $0 \nu \beta \beta$ decay operator in Eq. (11), $\boldsymbol{\sigma} \cdot \boldsymbol{\sigma} \tau^{+} \tau^{+}$, strongly connects spin-orbit partners. The omission also affects single- $\beta$ decay.

Despite these limitations, the shell model reproduces experimental single- $\beta$ decay rates well if one quenches the strength of the spin-isospin operator $\boldsymbol{\sigma}^{ \pm}$from its bare value $g_{A} \simeq 1.27$ [137-139]. (Of course, that is a big "if," which we address in detail in Sec. IV.) The quenching needed to agree with data is about $20 \%-30 \%$, and this small range is enough to describe Gamow-Teller transitions in nuclei with valence nucleons in the $p$ shell (a configuration space comprising the $0 p_{3 / 2}$ and $0 p_{1 / 2}$ single-particle orbitals, representing the valence shell for $A \sim 10$ ), the $s d$ shell (the $0 d_{5 / 2}, 1 s_{1 / 2}$, and $0 d_{3 / 2}$ orbitals, $A \sim 30$ ), the $p f$ shell (the $0 f_{7 / 2}, 1 p_{3 / 2}, 1 p_{1 / 2}$ and $0 f_{5 / 2}$ orbitals, $A \sim 50$ ), and the space spanned by the $1 p_{3 / 2}, 1 p_{1 / 2}, 0 f_{5 / 2}$, and $0 g_{9 / 2}$ orbitals, for use near $A=70$. With roughly the same quenching the model also reproduces the $2 \nu \beta \beta$ decay rate of the only emitter in this mass region, ${ }^{48} \mathrm{Ca}$. One shell model success, in fact, was the accurate calculation of the $2 \nu \beta \beta$ decay half-life of ${ }^{48} \mathrm{Ca}[140,141]$ before it was measured ${ }^{2}$ [143]. The shell model also reproduces other $2 \nu \beta \beta$ decay half-lives provided it uses a quenching factor appropriate for the configuration space and interaction [48, 49, 82, 134, 144, 145]. It requires a similar quenching to reproduce magnetic moments and transitions [146-148], which, like $\beta$ decay, involve the spin operator.

The reliability of the shell model depends on the effective interaction $H_{\text {eff }}$ as well as the configuration space. The starting point for $H_{\text {eff }}$ is usually the bare nucleonnucleon interaction, which is fit to two-nucleon scattering data (and discussed in a bit more detail in Sec V C). Then, many-body perturbation theory is used to obtain a configuration-space-only Hamiltonian that takes the effects of the inert core and neglected single-particle orbitals into account [149]. Finally, phenomenological corrections, mainly in the monopole part of the interaction (the part responsible for changes in single-particle-like excitations with increasing $A$ ) are usually made to improve the agreement with experimental data [121, 124, 125]. This last step in the construction of $H_{\text {eff }}$ severely restricts our ability to quantify the theoretical uncertainty associated with $0 \nu \beta \beta$ matrix-elements. The usual fallback - comparing the results of calculations that use equally reasonable effective Hamiltonians - leads to variations off $10 \%-20 \% \quad[54,108,134]$.

As Fig. 5 shows, shell-model $0 \nu \beta \beta$ matrix elements are usually smaller than all others. We discuss possible reasons in Sec. III F.

\section{B. The QRPA and Some of Its Variants}

The other major thrust over the past 30 years has been the development of the charge-changing quasiparticle random phase approximation (QRPA). This approach is a generalization of the ordinary random phase approximation (RPA), which has a long history both in nuclear physics and elsewhere [150, 151]. The method can be

\footnotetext{
2 The NEMO-3 collaboration has reported a longer half-live than was measured previously, resulting in a deviation of about two $\sigma$ [142] from the shell-model prediction. If this new value is confirmed, the shell model would overestimate the ${ }^{48} \mathrm{Ca} 2 \nu \beta \beta$ matrix element.
} 
derived in a number of ways. One is through an approach we take up in more detail later, the generator coordinate method (GCM). Suppose one has solved the Hartree-Fock equations, yielding a set of $\mathcal{A}$ occupied orbitals $\left|\varphi_{i}\right\rangle$ that when put together form the best possible Slater determinant $\left|\psi_{0}\right\rangle$, and has then constructed another set of "nearby" non-orthogonal Slater determinants $|\psi(z)\rangle$, each with $\mathcal{A}$ occupied orbitals of the form $\chi_{i}(z)$ :

$$
\left|\chi_{i}(z)\right\rangle=\left|\varphi_{i}\right\rangle+\sum_{j=\mathcal{A}+1}^{\mathcal{N}_{o}} z_{i j}^{*}\left|\varphi_{j}\right\rangle
$$

where $\mathcal{N}_{O}$ is the number of orbitals included in the calculation. The RPA ground state $|\mathrm{RPA}\rangle$ is the (continuous) superposition of the $|\psi(z)\rangle$ that minimizes the energy in the limit that the $z_{i j}$ are small. In that limit, the minimization process becomes equivalent to the solution of the Schrödinger equation for a multi-dimensional harmonic oscillator, in which the $z_{i j}$ play the role of creation operators [152]. Along with the ground state one finds a set of "one-phonon" normal modes, one-particle onehole excitations that are the only states that can be connected to the ground state by a one-body operator. The transition strengths to those states automatically satisfy energy-weighted sum rules.

For application to single- $\beta$ and $\beta \beta$ decay, the RPA must be modified in two ways. First, it must be "chargechanging," that is, if $\left|\varphi_{i}\right\rangle$ in Eq. (19) is a neutron orbital then at least some of the $\left|\varphi_{j}\right\rangle$ must be proton orbitals. That condition guarantees that the one-phonon excited states have components with one more proton and one less neutron than the ground state. Second, it must include the physics of pairing, which one can add by replacing the Hartree-Fock state with the BCS or Hartree-Fock-Bogoliubov (HFB) quasi particle vacuum, and the nearby Slater determinants with nearby quasiparticle vacua [153]. The result is the charge-changing (or proton-neutron) QRPA.

In single- $\beta$ decay QRPA excitations of the initial state produce final states. In $\beta \beta$ decay, they produce intermediate states in Eqs. (7) and (14) and one must carry out two separate QRPA calculations, one based on the initial nucleus as the BCS vacuum, and a second on the final nucleus. (The closure approximation is not required.) In this way one obtains two different sets of states in the intermediate nucleus, one of which must be expressed in terms of the other. The overlaps that are needed to do that require approximations beyond those in the QRPA. We will return to this subject in Sec. V B 3.

The main advantage of the QRPA in comparison to the shell model is the number of single-particle orbits that can be included in the calculation. As we mentioned in Sec. III A, shell-model configuration spaces are usually based on a few single-particle orbitals, most often in one major oscillator shell. In most QRPA calculations, all the orbitals within one or two oscillator shells of the Fermi surface are treated explicitly, with those further below assumed to be fully occupied and those further above

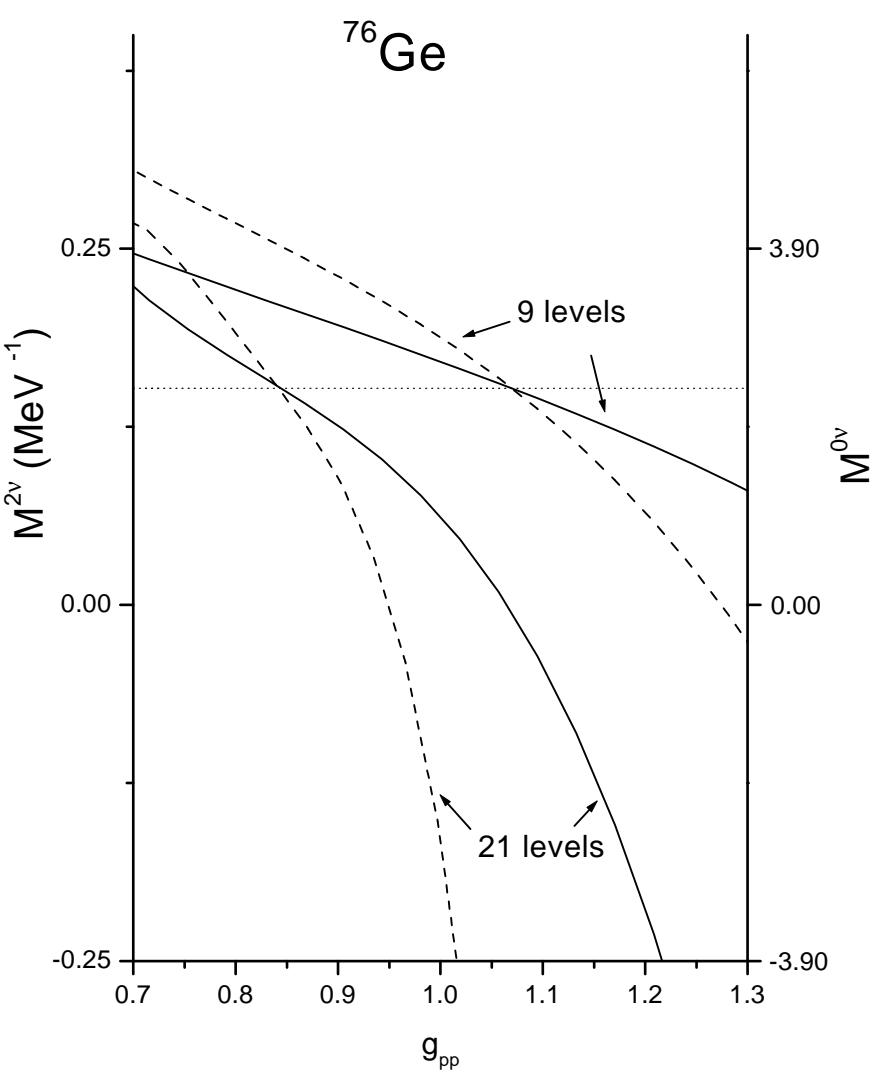

FIG. 6. Matrix elements $M_{G T}^{2 \nu}$, (left scale, dashed lines) and $M^{0 \nu}$, (right scale, solid lines) for the $2 \nu \beta \beta$ and $0 \nu \beta \beta$ decay of ${ }^{76} \mathrm{Ge}$, as a function of the strength of the proton-neutron interaction $g_{p p}$ for QRPA calculations in configuration spaces consisting of 9 and 21 single-particle orbitals. The dotted horizontal line is at the measured value of the $M_{G T}^{2 \nu}$. Figure taken from Ref. [154].

completely empty. One QRPA calculation [117], albeit a particularly demanding one, actually included all levels between 0 and $60 \mathrm{MeV}$, with no inert core.

Being able to handle many orbitals guarantees, for example, that the $\beta^{-}$and $\beta^{+}$strengths obey the "Ikeda sum rule" [see Eq. (20) in Sec. IV]. But the price for such large single-particle spaces in the QRPA is a restricted set of correlations. As a result, one cannot expect a really accurate calculation without compensating for the method's limitations by modifying the effective nucleonnucleon interaction used to generate the nuclear states. As in the shell model, the original interaction is typically a realistic nucleon-nucleon potential, one adapted to the QRPA configuration space through many-body perturbation theory [149]. The interaction is modified more severely than in the shell model, however, usually independently in the particle-hole and pairing channels. Practitioners often renormalize the strengths of the interaction in the proton-neutron particle-hole $J^{\pi}=1^{+}$ and $2^{-}$channels by $10 \%$ or so to properly reproduce the energies of the Gamow-Teller and spin-dipole giant res- 
onances, altering both the $2 \nu \beta \beta$ and $0 \nu \beta \beta$ matrix elements somewhat. It is the proton-neutron pairing interaction that is most important, however. Its strength, often called $g_{p p}$, is also changed by only about $10 \%$, but that modest adjustment usually has a large effect on the $\beta \beta$ matrix elements. Figure 6 shows a typical plot of the $2 \nu \beta \beta$ and $0 \nu \beta \beta$ matrix elements versus $g_{p p}$; the strong suppression near the nominally correct value of that parameter, $g_{p p}=1$, is clear not only in the $2 \nu \beta \beta$ matrix element, where it is dramatic, but also in the $0 \nu \beta \beta$ matrix element. The usual procedure in the QRPA is to fix the value of $g_{p p}$ so that the measured rate of $2 \nu \beta \beta$ decay is correctly reproduced [154]. Then the same value is used to predict the rate of $0 \nu \beta \beta$ decay. As Fig. 6 shows, this procedure almost eliminates the dependence of the $0 \nu \beta \beta$ matrix elements on the number of single-particle levels in the configuration space.

The reason for the sensitivity to the proton-neutron pairing strength has been the subject of many investigations [155]. Some of it is easy to understand: in the isoscalar channel, for example, proton-neutron pairing mixes proton-neutron spin-one pairs, which are like in-medium deuterons, into the usual condensate of likeparticle spin-zero pairs. The Gamow-Teller single- $\beta$ operator, which plays a role in both the $2 \nu \beta \beta$ matrix element in Eq. (14) and the $0 \nu \beta \beta$ matrix element in Eq. (11), connects the two kinds of pairs, altering the matrix elements. (For a simple discussion of why they shrink rather than grow as a result, see Ref. [156].) But some of the sensitivity can be an artifact of the QRPA. When the parameter $g_{p p}$ is made large enough the method breaks down because of an impending phase transition from the like-particle pairing condensate to a "deuteron-like" condensate. In the region before this (non-existent) transition, the sensitivity to $g_{p p}$ is unrealistically high.

Several groups have tried to modify the QRPA to cure this behaviour. The usual approach is the "renormalized QRPA" (RQRPA) [157, 158]. The idea is that the breakdown is due to the violation of the Pauli exclusion principle by the small-amplitude approximation at the heart of the QRPA, and that the violation can be remedied by modifying the BCS vacuum on which the QRPA phonons are based. In the "fully renormalized" version of Ref. [158] some terms are kept beyond lowest order in the small-amplitude expansion as well. These procedures succeed in avoiding the infinities that mark the breakdown of the ordinary QRPA, but may overcompensate by eliminating all traces of the approximate phase transitions that really take place in solvable models, where the methods have been benchmarked $[159,160]$. In realistic calculations, these methods alter the QRPA matrix elements by noticeable but not large amounts [53]. More comprehensive and systematic modifications to the QRPA have been proposed; we discuss some of them in Sec. VB 3 .

Finally, it is possible to calculate $0 \nu \beta \beta$ matrix elements, in the closure approximation, in the like-particle QRPA rather than the charge-changing version. With closure, any set of intermediate states that form a partition of the identity operator will do. To apply the likeparticle QRPA, one chooses these intermediate states to lie in the nucleus with two-more protons (or two-less neutrons) than the initial nucleus. Though such excitations do not conserve particle number, or even charge in the case of two-proton addition, they can be represented as two-like-quasiparticle excitations of the initial state. So far, however, only one author $[161,162]$ has applied the QRPA in this way.

Turning at last to QRPA-produced $\beta \beta$ matrix elements: Their most notable feature is that they are almost uniformly larger than shell model matrix elements (see Fig. 5), whether or not a renormalized version of QRPA is used. We discuss the reasons in Sec. IIIF.

\section{Energy-Density Functional Theory and the Generator-Coordinate Method}

The term Energy-Density Functional (EDF) theory refers at its most basic level to the process of minimizing an energy functional $\mathcal{E}[\rho, \boldsymbol{s}, \boldsymbol{j}, \ldots]$ with respect to local and semi-local densities such as the number density $\rho$, the spin density $\boldsymbol{s}$, the current density $\boldsymbol{j}$, etc. [163]. The functional $\mathcal{E}[\rho, \boldsymbol{s}, \boldsymbol{j}, \ldots]$ is the minimum possible value for the expectation value of the Hamiltonian when the densities are constrained to have particular values. Once the functional is obtained, minimizing it with respect to its arguments provides the exact ground-state energy and densities, as Hohenberg and Kohn originally showed [164]. Moreover, the minimization can be formulated so that it looks like mean-field theory with one-body potentials and orbitals, via the Kohn-Sham procedure [165]. The independent particle or quasiparticle wave functions that result have no meaning beyond supplying the correct energy and densities. In nuclear physics, approximations to the functional $\mathcal{E}$ usually derive from the Hartree-Fock or HFB energy asociated with a "density-dependent twobody interaction" of the Skyrme [166], Gogny [167] or relativistic Walecka [168] type, sometimes with additional modifications. The parameters of the interaction or functional are then fit to ground-state properties - masses, radii, etc. - in a variety of nuclei and used without alteration all over the nuclear chart. The method can be extended to EDF-based RPA or QRPA, which are adiabatic versions of time-dependent Kohn-Sham theory. Indeed, that approach was used profitably in the $\beta \beta$ decay calculations of Ref. [117].

The problem at present is that the Skyrme, Gogny, or relativistic functionals $\mathcal{E}$, though they do a good job with collective properties such as binding energies, radii, and E2 transitions, are not close enough to the exact functional to work for all quantities in all nuclei ${ }^{3}$. Some-

\footnotetext{
${ }^{3}$ Nor should they be, even in principle. The Hohenberg-Kohn
} 
thing must be added to accurately describe nuclear properties, and the easiest way to add physics is to explicitly modify the Hartree-Fock or HFB wave functions so that they contain explicit correlations [169]. Adding correlations means mixing the wave-function-based and EDFbased approaches, however, opening the door to all kinds of inconsistencies [170]. The only way to remove these is to abandon the focus on functionals and return to a Hamiltonian. It is still an open question whether densitydependent Hamiltonians of the kind used in phenomenological calculations of isotopes along the nuclear chart can ever be made fully consistent.

The use of functionals together with explicit correlations is valuable nonetheless, particularly within the GCM. Although the method can be used in conjunction with the small-amplitude approximation to derive the RPA or QRPA (see Sec. III B) the need for it is greatest when deviations from a single mean field have large amplitudes and the QRPA breaks down. Large-amplitude fluctuations are often associated with collective correlations. Shapes with significantly different degrees of deformation often must coexist, making the QRPA inapplicable. The same is sometimes true of pairing gaps. And both deformation and pairing involve the simultaneous motion of many nucleons in many orbitals, some of which may be outside shell-model spaces. The large single-particle spaces in EDF-based work and the mixing of mean fields in the GCM make it possible for these collective correlations to be fully captured.

The GCM with EDFs has several steps [169]:

1. Choose one or more "collective operators" $\hat{O}_{i}$. The most commonly chosen is the axial quadrupole operator $r^{2} Y_{20}$. Operators that reflect non-axial deformation and pairing gaps are other common choices.

2. Carry out repeated mean-field calculations with the expectation values of the $\hat{O}_{i}$ constrained to many different values (approximating a continuum of values). In other words, find the Slater determinants or $\mathrm{HFB}$ vacua that minimize $\mathcal{E}$ under the constraint that the $\left\langle\hat{O}_{i}\right\rangle$ take on particular sets of values.

3. Project the resulting mean-field states onto states with well defined angular momentum, particle number, and whatever other conserved quantity the mean-field approximation does not respect.

4. Use the resulting non-orthogonal states as a basis in which to "diagonalize the Hamiltonian."

The last phrase is in quotation marks because although the EDF is associated with one or more densitydependent Hamiltonians (often separate ones for the

theorem does not imply that the functional has the same form in all nuclei. particle-hole and pairing channels), the theory says nothing about how to evaluate matrix elements of the density or of density-dependent operators between different Slater determinants. The most sensible and commonly used procedure is to replace the density by the transition density, but the approach has been shown to produce ill-defined singularities when examined closely [170172] and no alternative has gained currency. Some functionals have fewer problems than others, however, and the Gogny and relativistic functionals, which are based strictly on density-dependent Hamiltonians, both allow stable results, provided one does not push numerical accuracy too far.

The applications to $0 \nu \beta \beta$ decay have been relatively few so far. Reference [173] contains the first, with the Gogny functional and axial quadrupole moment $\left\langle r^{2} Y_{20}\right\rangle$ as the generator coordinate. In Ref. [120], fluctuations in particle number were added as coordinates, leading in a modest enhancement of the matrix elements from the richer pairing correlations. References [118, 174] used the axial quadrupole moment as a coordinate in conjunction with a relativistic functional, and Ref. [119] added octupole deformation in the decay of the rare-earth nucleus ${ }^{150} \mathrm{Nd}$. The most obvious feature of all these results is that the $0 \nu \beta \beta$ matrix elements are larger than those of the shell model, and usually larger than those of the QRPA as well. The reason seems to be missing correlations in the GCM ansatz. At first these were thought to be non-collective [175], but recent work [176] strongly suggests that most of them come from the isoscalar pairing that we encountered when discussing the QRPA in Sec. III B. Quite recently, Ref. [177] included the isoscalar pairing amplitude as a generator coordinate together with a Hamiltonian in one or two oscillator shells rather than an EDF. That coordinate allows the GCM to reproduce shell-model results quite well. The Hamiltonianbased GCM can easily be extended to larger spaces, and we discuss plans to do so in Sec. IIIF.

\section{The Interacting Boson Model}

The strength of the shell model is the inclusion of all correlations around the Fermi surface and that of the GCM is a careful treatment of collective motion. The IBM aims to share both these strengths and describes excitation spectra and electromagnetic transitions among collective states up to heavy nuclei [178]. The cost, perhaps, is more abstract degrees of freedom than nucleons and a more phenomenological approach to nuclear structure.

The IBM leverages the algebra of boson creation and annihilation operators to provide simple Hamiltonians that generate complex and realistic collective spectra. In its original form (IBM-1) [179], the degrees of freedom are $\mathcal{N}$ bosons (where $\mathcal{N}$ is usually half the number of nucleons in the shell-model configuration space), each of which can be in six positive parity states: an 
angular-momentum-zero state (in which case the boson is labeled " $s ")$ and five angular-momentum-two states (in which case it is labeled " $d_{\mu}$," with $\mu$ the magnetic projection). The Hamiltonian is usually a combination of one- and two-boson scalar operators, of the form $s^{\dagger} s,\left(d^{\dagger} \tilde{d}\right)^{0},\left(\left[s^{\dagger} \tilde{d}\right]^{2}\left[d^{\dagger} \tilde{d}\right]^{2}\right)^{0}$, etc., where $\tilde{d}_{\mu} \equiv(-1)^{\mu} d_{-\mu}$ and the superscripts denote the angular momentum to which the operators inside the corresponding parentheses or square brackets are coupled. In the IBM-2 [178], which is used to study $\beta \beta$ decay, there are separate $s$ and $d$ boson states for neutrons and protons.

The IBM is appealing because it has clear connections to both the shell model and the collective model of Bohr and Mottelson [180]. On the one hand, the bosons represent nucleon pairs and on the other quadrupole phonons. Because the first correspondence is hard to make precise, Hamiltonians and effective operators are usually determined by fits to data rather than by mappings from the shell model [181]. There are no data on $0 \nu \beta \beta$ matrix elements, however, and the associated operators therefore must be derived from the shell model, at least approximately. The mapping for doing so is described in Ref. [182]. It is approximate because it involves only two- and four-nucleon states (which are mapped to one- and twoboson states) and a schematic surface-delta shell-model interaction that is inconsistent with the phenomenological boson interaction.

The $0 \nu \beta \beta$ matrix elements produced by the IBM generally lie between those of the EDF/GCM and those of the shell model, and for several isotopes are close to the QRPA values. This result is perhaps surprising because the IBM is supposed to be a collective approximation to the shell-model in one harmonic oscillator shell. The behavior may be connected to the truncation of the set of all possible bosons to a single set of like-particle $s$ and $d$ objects, but that is just a guess; the subject needs to be investigated more thoroughly.

\section{E. Other Approaches}

We will do little more than mention a couple of other methods that have been used to calculate $\beta \beta$ matrix elements. Neither gives results that are startlingly different from those of other more modern and sophisticated approaches.

References [183-186] describe calculations with projected HFB states in a valence space of between one and two shells, with a schematic paring plus quadrupole Hamiltonian. The method itself is a simpler version of the GCM, which diagonalizes Hamiltonians in a space built from many projected HFB states.

References [187, 188] describe calculations in the pseudo-SU(3) model of the $0 \nu \beta \beta$ matrix elements for ${ }^{150} \mathrm{Nd}$, for the very heavy nucleus ${ }^{238} \mathrm{U}$, and for a few other deformed isotopes. The model takes advantage of a dynamical symmetry in the deformed Nilsson basis to construct states that should be similar to those ob-
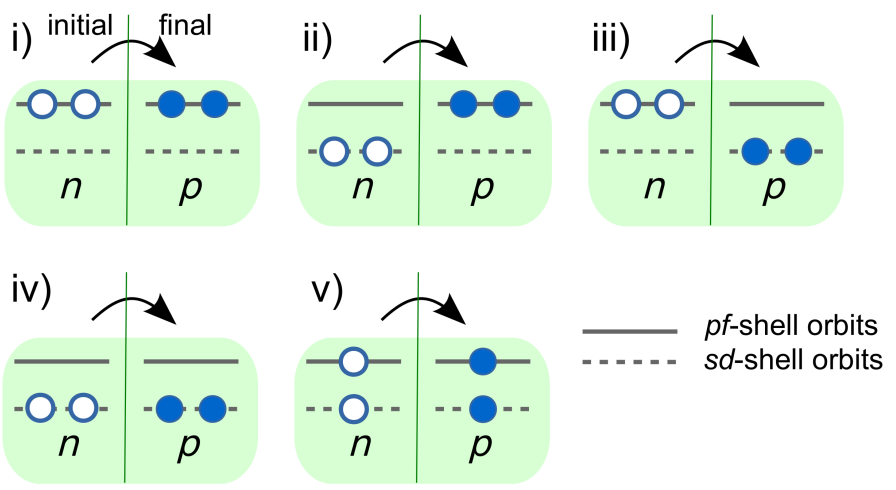

FIG. 7. Schematic contributions to shell model matrix elements from various parts of configuration space, for neutron (n) and proton (p) orbitals. Diagram i) shows the contributions from within the standard shell model configuration space. Diagrams ii)-iv) illustrate the contributions from $2 \mathrm{p}-$ $2 \mathrm{~h}$ excitations beyond this space, while diagram $\mathrm{v}$ ) shows contributions from $1 \mathrm{p}-1 \mathrm{~h}$ excitations. Focus is on the $p f$ (standard configuration space) and $s d$ (additional single-particle orbitals) shells used to study ${ }^{48} \mathrm{Ca}$, but the competition between diagrams ii)-iv) and v) is general. Figure taken from Ref. [114].

tained from number- and angular-momentum-projected HFB calculations of the kind discussed in the previous paragraph and in Sec. III C.

\section{F. Tests and Comparisons}

Although we have mentioned the strengths and weaknesses of the many-body approaches most commonly used to calculate $0 \nu \beta \beta$ matrix elements, it is worth trying to compare them more carefully. How important are all the correlations included in the shell model but neglected in the QRPA, GCM, and IBM? What about the large configuration spaces in which the GCM and QRPA work, but which the shell model and IBM cannot handle? A few publications address these matters and we discuss them here.

References $[114,145,189]$ have studied the effects of enlarging the configuration space in the shell model. The tentative conclusion is that allowing up to one or two particles to occupy a few selected orbitals beyond the original configuration space can have a strong effect on $2 \nu \beta \beta$ matrix elements (in particular if these orbitals are the spin-orbit partners of orbitals in the original configuration space [145]), but the effects on $0 \nu \beta \beta$ matrix elements are more moderate. The reason is that two distinct kinds of correlations compete, as illustrated in Fig. 7, taken from Ref. [114]. On one hand, pairing-like excitations to the additional orbitals enhance the $0 \nu \beta \beta$ matrix elements [189] (diagrams ii, iii and iv in Fig. 7); on the other hand, particle-hole excitations (1p-1h) to the additional orbitals, which in principle require less en- 
ergy than pairing-like excitations $(2 \mathrm{p}-2 \mathrm{~h})$, tend to reduce the matrix elements (diagram $\mathrm{v}$ in Fig. 7). The net result appears to be a moderate increase, generally by less than $50 \%$. A careful calculation of the matrix element for ${ }^{48} \mathrm{Ca}$ [114] that extended the configuration space from four to seven single-particle orbitals found about a $30 \%$ enhancement. That agrees reasonably well with results of calculations that include the effect of extra orbitals perturbatively: a $75 \%$ increase in the matrix element of ${ }^{48} \mathrm{Ca}$ [190], along with a $20 \%$ increase in ${ }^{76} \mathrm{Ge}$, and $30 \%$ increase in ${ }^{82} \mathrm{Se}$ [191]. (In perturbation theory configuration spaces even larger than those in QRPA and GCM calculations can be included.) Though the enhancement is greater in perturbation theory, the final matrix elements in Refs. [114] and [190] differ by only $20 \%$.

Other publications $[108,133,192]$ have reported studies of the correlations included in the shell-model but not in the QRPA. Nuclear many-body states can be classified by a "seniority" quantum number $s$ that labels the number of nucleons not in correlated neutronneutron and proton-proton $J^{\pi}=0^{+}$pairs. Fully paired $s=0$ states contribute the major part of $\beta \beta$ matrix elements $[108,133]$, a fact that is consistent with the finding that the additional pairing (2p-2h-like) correlations from extra orbitals enhance the matrix element. The states with broken pairs (seniority $s>0$ ) contribute with opposite sign, shrinking matrix elements [108, 133]. An overestimate of pairing correlations, i.e. of the $s=0$ component in the nuclear states, thus leads to an overestimate of the matrix elements themselves. QRPA correlations in spherical nuclei include states with seniority $s=4,8,12$ but not those with $s=6$ and 10, and QRPA matrix elements could be too large for that reason. Although the contributions of states with $s=6$ and 10 are relatively small in shell model calculations [192], Ref. [193] noted that when shell model states are forced to have the same seniority structure as QRPA states, the resulting shellmodel matrix elements grow, implying that the shortage of broken pairs in the QRPA makes its matrix elements too large. On the other hand, Ref. [194] observed that when the QRPA is applied within the small shell-model configuration space (which is not a natural space for the QRPA) the resulting matrix elements are similar to those of the shell model, suggesting that the shell-model matrix elements are about $50 \%$ too small. These issues are still unresolved.

The shell model's ability to incorporate all correlations induced by the nuclear interaction, at least in a small model space, allows it to benchmark the GCM as well as the QRPA, and to tease out the most important kinds of correlations for $0 \nu \beta \beta$ decay. Reference [175] compared shell model and EDF/GCM matrix elements for the decay of a wide range of calcium, titanium and chromium isotopes. These nuclei are not candidates for a $0 \nu \beta \beta$ decay experiment, but their decay matrix elements can still be calculated, allowing one to perform a systematic study in medium-mass nuclei. The calculations of Ref. [175] were in the full $p f$ shell, a useful testbed because it

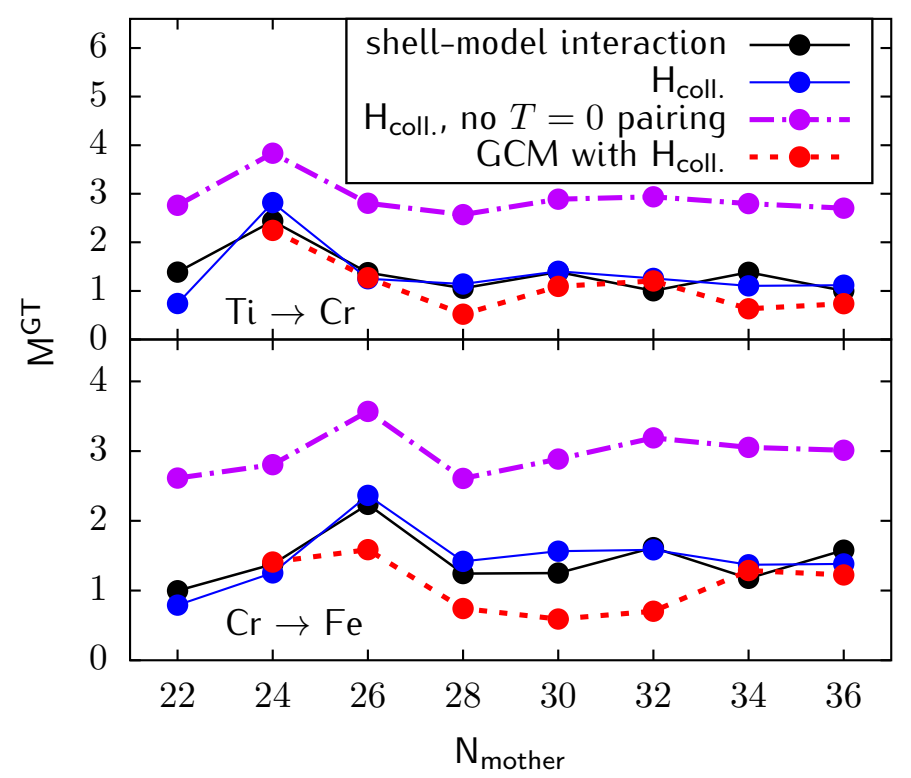

FIG. 8. Gamow-Teller part $\left(M_{G T}^{0 \nu}\right)$ of the nuclear matrix elements for the decay of titanium (top) and chromium (bottom) isotopes as a function of neutron number. Shell model results obtained with the KB3G shell model interaction [196] (black, solid lines) are compared with those produced by a collective Hamiltonian, $H_{\text {coll. }}$, (blue, solid lines) and with GCM matrix elements produced by the same collective Hamiltonian, with the isoscalar-pairing amplitude as a generalized coordinate (red, dashed lines). Shell model results produced by the collective Hamiltonian without isoscalar $(T=0)$ pairing are also shown (purple dashed-dotted lines). Figure adapted from Ref. [176].

includes all spin-orbit partners in the one-shell configuration space, removing one of the shortcomings of the shell model in heavier nuclei (see Sec. III A). In addition, both the shell model and EDF theory with the GCM describe the spectra of nuclei in this region quite well [121, 195]. The main finding of Ref. [175] is that the EDF-based GCM and the shell model produce similar matrix elements when spherical EDF configurations are kept and shell-model configurations are restricted to those with $s=0$. It is only when higher seniority components, which include correlations beyond pairing, are permitted that the shell model matrix elements decrease. The reduction is much larger than that which can be induced by including axial deformation as one of the coordinates in the EDF-based GCM. The inclusion of high-seniority states in the shell model but not the EDF-based GCM thus explains the results in Fig. 5, where shell model matrix elements are always smaller than those produced by EDF theory.

What is the nature of the higher-seniority correlations that reduce matrix elements? Quadrupole correlations, induced by deformation, can play a role, particularly when the initial and final nuclei are deformed by different amounts [173, 197, 198]. But the QRPA and shell model 
agree that isoscalar pairing correlations usually play an even more crucial role $[155,156,176]$. Figure 8 , based on work in Ref. [176], compares shell-model matrix elements with and without isoscalar pairing correlations. Put more precisely, it compares results produced by the full shellmodel Hamiltonian with those of an approximate collective Hamiltonian [128] from which isoscalar pairing can be excluded. The collective Hamiltonian reproduces the full results nearly perfectly. Without isoscalar pairing, however, the collective Hamiltonian greatly overestimates the matrix elements, always by about two units.

After noting the importance of isoscalar pairing, Ref. [176] demonstrated that the correlations missing from the GCM are nearly all of that kind. The paper tested a version of the GCM that works with Hamiltonians rather than EDFs. This shell-model based GCM and the shell model itself used the same Hamiltonian - the collective approximation - and the same configuration space; the shell model was thus the "exact" solution in the test because it diagonalized the interaction exactly. The result, shown in Fig. 8, was good overall agreement, provided the GCM included the isoscalar pairing amplitude as one of its generalized coordinates [177]. (Some small differences remain for decays involving closed shells because these isotopes have fewer collective correlations.) That step allowed the method to capture isoscalar pairing correlations quite well. GCM calculations in larger spaces do not yet include isoscalar pairing coordinate, but should be able to in the near future. The Hamiltonianbased GCM may thus be able to include both the large model spaces of the QRPA and the important shell-model correlations, without the drawbacks of either method.

Comparing the many-body methods suggests that with proper attention, each of them could obtain accurate matrix elements, which might well lie somewhere between the current predictions of the shell model and those of the EDF/GCM and QRPA. We will say more about how to improve and benchmark the different methods in the near future in Sec. V. First however, we turn to an issue that plagues all calculations in heavy nuclei and vitiates the idea that the matrix elements should be in the range spanned by current calculations: the over-prediction of single- $\beta$ and $2 \nu \beta \beta$ matrix elements, sometimes referred to as the " $g_{A}$ problem".

\section{THE $g_{A}$ PROBLEM}

\section{A. Systematic Over-prediction of Single- $\beta$ and $2 \nu \beta \beta$ Matrix Elements}

For nuclei close to stability up to mass number $A \sim 60$, calculations reproduce ground state properties, excitation spectra and electric moments and transitions well ${ }^{4}$

\footnotetext{
4 The moments and transitions require effective charges that one can obtain by treating collective states outside the configuration
}

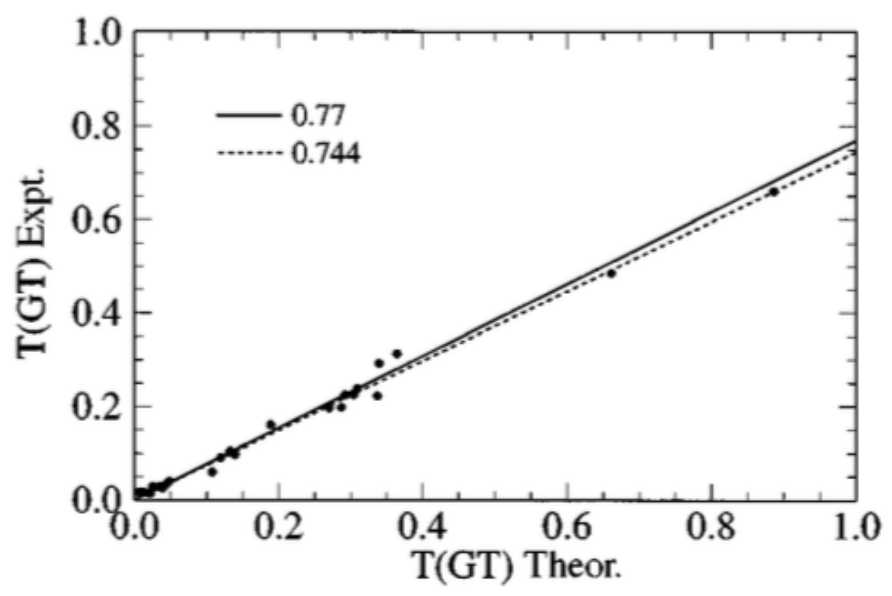

FIG. 9. Experimental versus theoretical strengths for a compilation of Gamow-Teller transitions in nuclei with mass number between about 40 and 50 . The dashed line is a fit of the theoretical results to data, and suggests that the effective value of $g_{A}^{\text {eff }}$ of the axial-vector coupling is $0.74 g_{A}$. Figure taken from Ref. [139].

$[121,124,125]$. They do not do as well with $\beta$-decay rates, at least not without a tweak. Figure 9, taken from Ref. [139], compares of experimental Gamow-Teller strengths - summed over certain low-lying states and scaled as described in that reference - for nuclei with mass number $A$ between about 40 and 50 versus the theoretical predictions. The calculated strengths are generally larger than the data, but if the Gamow-Teller operator $\boldsymbol{\sigma} \boldsymbol{\tau}$ is multiplied by 0.74 or, equivalently, if the axial coupling $g_{A} \simeq 1.27$ is replaced by an effective value $g_{A}^{\mathrm{eff}}=0.74 g_{A}$, then the calculations reproduce most of the experimental data quite well. A similar phenomenological correction, $g_{A}^{\text {eff }}=0.82 g_{A}$, brings predictions into agreement with data for nuclei with $A$ less than 16 [137]. For $A$ between about 16 and 40, a value of $g_{A}^{\mathrm{eff}}=0.77 g_{A}[199]$ works well, and a slightly larger phenomenological correction $g_{A}^{\mathrm{eff}}=0.68 g_{A}$ is preferred for $A$ between 60 and 80 [200]. That such a simple renormalization can largely eliminate a theoretical problem is a remarkable fact that has resisted attempts at explanation for several decades [146, 201-205].

The problem is not confined to $\beta$ decay or even electroweak operators. The total Gamow-Teller integrated strength is governed by the Ikeda sum rule [206]:

$$
\sum S_{G T}^{-}-S_{G T}^{+}=3(N-Z)
$$

where $S_{G T}^{\mp}$ is the total Gamow-Teller strength from the $\boldsymbol{\sigma} \tau^{+}$(neutron to proton) or $\boldsymbol{\sigma} \tau^{-}$(proton to neutron) operators, summed over all energies, and $N$ and $Z$ are

space in perturbation theory [128]. 


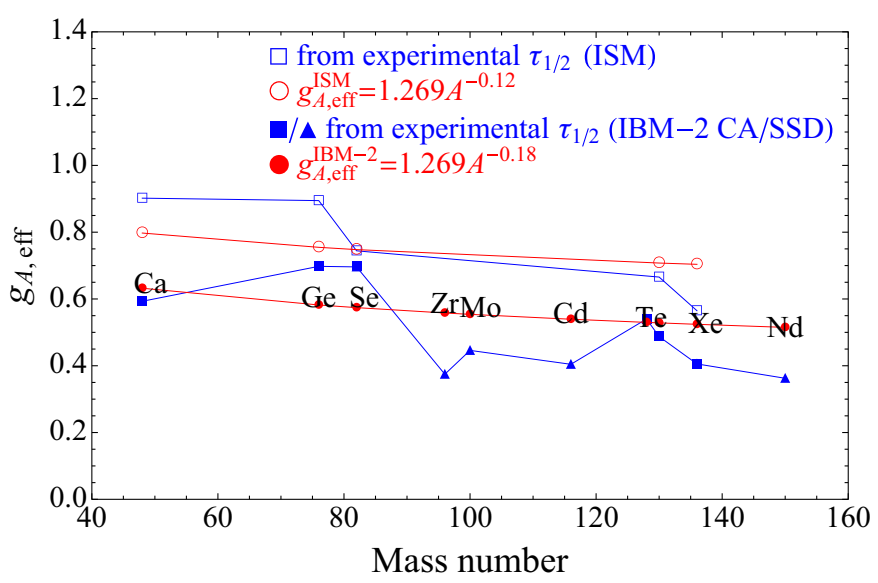

FIG. 10. Value of the effective strength of the $\boldsymbol{\sigma} \tau^{+}$operator, $g_{A}^{\text {eff }}$, required for shell model (ISM) and IBM calculations to reproduce the experimental matrix elements for several $2 \nu \beta \beta$ transitions, which are ordered by mass number. Figure taken from Ref. [109].

the initial nucleus's neutron and proton numbers. The sum rule is a simple consequence of commutation relations and must hold to the extent that neutrons and protons (as opposed, e.g., to $\Delta$-isobar excitations) are the only important nuclear degree of freedom. The summed strength can be extracted from charge-exchange experiments in which, for example, a proton is absorbed and a neutron is emitted, or a ${ }^{3} \mathrm{He}$ ion is absorbed and a triton emitted. The weak interaction plays no part in these reactions but they nevertheless measure Gamow-Teller strength because the cross-section at forward angles is determined by the transition matrix elements of the $\boldsymbol{\sigma} \tau^{ \pm}$ operators [207-209]. Experiments that can determine the sum $S_{G T}^{-}$of strength below about $50 \mathrm{MeV}$ report considerably less than $3(N-Z)$, typically about half that much [207]. $\left(S_{G T}^{+}\right.$is much smaller because stable nuclei usually have more neutrons than protons and proton-to-neutron transitions are thus Pauli blocked.) That amount would correspond to a "quenching" of the $\boldsymbol{\sigma} \tau^{+}$operator that is similar to what is needed for single- $\beta$ decay.

The agreement is strange. One experiment examines weak decay and the other tests nothing but the strong interaction. In one experiment, nature disagrees with complicated many-body calculations and in the other with a simple consistency requirement (though measured strength distributions are also smaller than calculations at low energies, demanding a quenching that is consistent with that used for $\beta$-decay [210-213]). Some chargeexchange experiments $[214,215]$ suggest that both discrepancies are due to $\boldsymbol{\sigma} \tau^{+}$strength that spreads out above the Gamow-Teller resonance, up to $50 \mathrm{MeV}$ or more of excitation energy. There is still no consensus about the suggestion, however, mainly because it is hard to distinguish spin-isospin strength from background at high energies [207, 209].
Given this comprehensive quenching of Gamow-Teller strength, it is not surprising that the " $g_{A}$ problem" also afflicts calculations of $2 \nu \beta \beta$ matrix elements. Figure 10, taken from Ref. [109], shows the values of $g_{A}^{\text {eff }}$ required to reproduce measured $2 \nu \beta \beta$ matrix elements for a selection of shell-model and IBM calculations (the latter of which are in the not-always-reliable $2 \nu \beta \beta$-decay closure approximation.) In both models $g_{A}^{\text {eff }}$ decreases with mass, approaching half of $g_{A}$ or less in the heavier nuclei. Although other sets of shell-model calculations show milder and less mass-dependent quenching [82, 134, 144] and the mass dependence might be due to the fixable omission of spin-orbit partners $[144,145]$, the main message of Fig. 10 is undeniable and its implication stark. $2 \nu \beta \beta$ decay rates, which are proportional to the fourth power of $g_{A}^{\text {eff }}$, are much smaller than shell-model and IBM predictions $^{5}$. If $0 \nu \beta \beta$ decay rates, to which the main contribution is $M_{G T}$ in Eq. (10), are smaller than predictions by a similar amount, next generation experiments will be significantly less sensitive than we currently expect; they will not be able to rule out the inverted hierarchy with a ton of material. Is $0 \nu \beta \beta$ decay really that quenched?

The answer depends on the source of the quenching, which is still unknown. We discuss possibilities and their implications next.

\section{B. Possible Causes and Implications for Neutrinoless Double-Beta Decay}

Over the years, theorists have suggested a wide range of sources for the quenching of Gamow-Teller strength. Almost all, in modern language, fall into one of two classes: nuclear many-body correlations that escape calculations [146, 202-204], and many-nucleon weak currents $[146,205]$. The former include short-range correlations, multi-phonon states, particle-hole excitations outside shell-model configuration spaces, etc. The latter stand for non-nucleonic degrees of freedom, i.e. $\Delta$-isobar excitations, in-medium modification of pion physics, partial restoration of chiral symmetry, etc. The consequences for $0 \nu \beta \beta$ decay depend on which of these two complementary sources is mostly responsible for the renormalization of the $\boldsymbol{\sigma} \boldsymbol{\tau}$ operator.

The reason that the relative quenching of $2 \nu \beta \beta$ and $0 \nu \beta \beta$ matrix elements can depend on the source is that there are marked differences between the two processes, even though both involve two virtual single- $\beta$ decays. The virtual neutrino that is emitted and reabsorbed in $0 \nu \beta \beta$ decay makes the average momentum transfer from nucleons to leptons at each vertex much higher than in $2 \nu \beta \beta$ decay. If the neutrinos actually emerge from the decay, the momentum and energy transfer are constrained

\footnotetext{
5 As we discussed earlier, the QRPA fits the strength of isoscalar pairing so as to reproduce $2 \nu \beta \beta$ decay rates.
} 


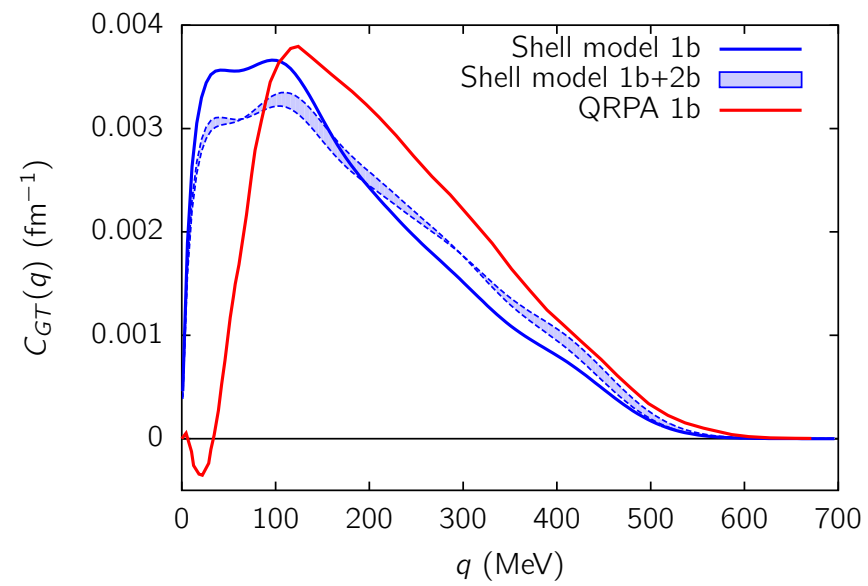

FIG. 11. Normalized momentum transfer distribution of the Gamow-Teller part of the nuclear matrix element of ${ }^{136} \mathrm{Xe}$. The solid curves are with one-body currents only, in the shell model (blue) and QRPA (red). The shaded area includes twobody contributions in the shell model. Data are taken from Ref. [217] (shell model) and Ref. [218] (QRPA).

by the $Q$-value of the transition, which is on the order of 1 $\mathrm{MeV}$. If only electrons are emitted, however, the average momentum is about $100 \mathrm{MeV}$, a scale set by the average distance between the two decaying neutrons. Fig. 11 presents the contribution of different momentum transfers $q$ to the nuclear matrix element produced by the shell model and QRPA, for the representative mother nucleus ${ }^{136} \mathrm{Xe}$. The momentum-transfer distribution is similar in the two calculations. Although the QRPA distribution is shifted to higher $q$, probably because of the larger configuration space, it falls off slowly in both cases so that several hundred $\mathrm{MeV}$ are transferred with reasonable probability. The higher momentum transfer means that the first virtual $\beta^{-}$transition in $0 \nu \beta \beta$ decay can excite virtual intermediate nuclear states with all spins and parities, not just the $0^{+}$or $1^{+}$intermediate states that contribute to $2 \nu \beta \beta$ decay. If the spin-isospin renormalization depends on the momentum transfer or multipolarity of the intermediate states, the large quenching needed to correctly predict single- $\beta$ Gamow-Teller and $2 \nu \beta \beta$ decay rates may not be needed for $0 \nu \beta \beta$ decay. Although experimentalists are trying to test the momentum-transfer and multipolarity dependence of quenching [216], the experiments are difficult and the existing data inconclusive.

In the search for the cause of quenching, complex correlations that calculations do not capture have long been a suspect. Reference [202] proposed in 1982 that twoparticle-two hole excitations to orbitals outside shellmodel configuration spaces or beyond QRPA correlations shift the Gamow-Teller strength to high energies. (The Ikeda sum rule requirement means that strength does not appear or disappear, but rather moves.) Nuclearstructure models miss this effect and therefore need to quench the low-energy strength. The authors of

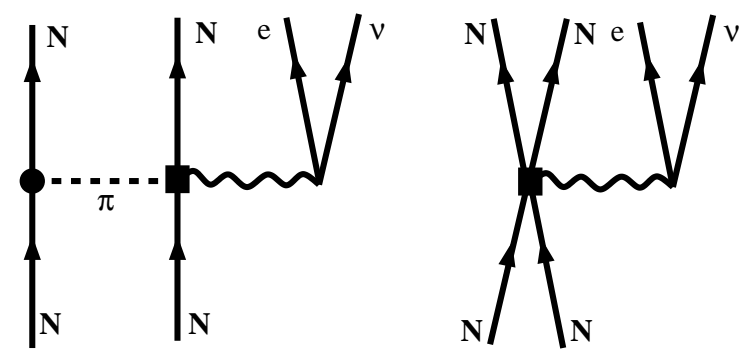

FIG. 12. Diagrams for the $\chi$ EFT two-nucleon currents most important for $\beta$-decay.

Refs. [146, 203] made a similar argument, and Ref. [147] proposed that about two thirds of the spin-isospin quenching comes from missing particle-hole configurations. The authors of Ref. [204] argued slightly differently, suggesting that because $\boldsymbol{\sigma} \boldsymbol{\tau}$ operates at all internucleonic distances, its matrix elements should be affected not only by the long-range (low-energy) correlations included e.g. in shell-model states, but also by short-range (high-energy) correlations, which are not included. They went on to argue that shell-model GamowTeller strength should be quenched consistently with the roughly $30 \%$ depletion of single-particle occupancies needed to reproduce electron scattering data [219] because both kinds of quenching reflect the same inability of the shell model to include short-range correlations.

More recently, two studies have tried to use many-body perturbation theory [149] to quantify the effect of missing correlations on the $\boldsymbol{\sigma} \boldsymbol{\tau}$ operator in the shell model. Reference [220] reported a 20\% reduction of Gamow-Teller strength for nuclei whose valence nucleons are in the $s d$ and $p f$ shells; the result agrees well with phenomenological fits to experimental strength. In heavier systems the authors found a much stronger reduction, as large as a $60 \%$ in ${ }^{100} \mathrm{Sn}$; that resut is in reasonable agreement with the trend shown in Fig. 10. The degree of renormalization varies by only a few percent up to momentum transfers of about $100 \mathrm{MeV}$, suggesting similar quenching of $2 \nu \beta \beta$ and $0 \nu \beta \beta$ matrix elements. Reference [191] studied $\beta \beta$ decay within a similar perturbative framework. While the method required the closure approximation and so could say relatively little about $2 \nu \beta \beta$ decay, it produced about a $20 \%$ enhancement of the $0 \nu \beta \beta$ matrix element in ${ }^{76} \mathrm{Ge}$ and a $30 \%$ enhancement in ${ }^{82} \mathrm{Se}$. These results agree with the tendency of the shell model to increase $0 \nu \beta \beta$ matrix elements when configuration spaces are enlarged slightly $[114,189]$, and argue against any suppression of $0 \nu \beta \beta$ decay. Once more, however, the argument is not conclusive: First-order one-particle one-hole excitations strongly suppress the matrix element and it just so happens that higher-order terms tend to counteract the suppression. But it is not at all clear whether or how fast the perturbative expansion converges, and neglected terms could have large effects.

Non-nucleonic degrees of freedom, manifested as many- 
nucleon currents in models without explicit $\Delta$-isobars and pions, are also a long-term suspect in the search to explain quenching (see e.g. Ref. [146] and references therein). Reference [147] concluded that only about one third of the phenomenological quenching is due to mesonexchange currents, most of which involved the $\Delta$-isobar. The currents in use at the time were based on models with no systematic power counting, however, and so the uncertainty in the result is very large. The situation has improved with the advent of $\chi \mathrm{EFT}$, which relates electroweak currents to nuclear interactions [221-223]. Several studies have exploited the relation in nuclei with $A \lesssim 10$, and it is now clear that the two-nucleon currents are necessary in precise calculations of weak [224-227] and electromagnetic $[228,229]$ transition rates. The effects in these nuclei range from a few percent to about $40 \%$ and depend significantly on nuclear structure.

Reference [217] applied many-nucleon currents, shown in Fig. 12 , to the single- $\beta$ and $\beta \beta$ decay of medium-mass nuclei. The authors reduced the two-body current operators to effective one-body operators by normal ordering with respect to a spin-isospin-symmetric Fermi-gas reference state. They found that the single- $\beta$ and $2 \nu \beta \beta$ matrix elements were reduced by an amount corresponding to $g_{A}^{\text {eff }} \sim(0.7-0.8) g_{A}$. Most of the quenching comes from the long-range one-pion-exchange current (depicted in the left-hand diagram in Fig. 12), which includes contributions from the $\Delta$-isobar. Uncertainty in the $\chi \mathrm{EFT}$ couplings that appear in the two-nucleon currents, especially in the coefficient of the contact term (depicted in the right-hand diagram in Fig. 12), leads to a significant uncertainty in the quenching, and even allows a slight enhancement.

Interestingly, the corresponding reduction in $0 \nu \beta \beta$ matrix elements turned out to be about 30\% [217] as well, much less than one would get by squaring the renormalization factor from single- $\beta$ decay, as one does to get the quenching of $2 \nu \beta \beta$ matrix elements. Later corrections to the currents $[230,231]$ did not change this fact. The two-body currents have a smaller effect at high momentum transfer, softening the quenching of $0 \nu \beta \beta$ matrix elements. QRPA calculations that used the same currents obtained even less quenching (about 20\% [218]) partly because the average momentum transfer is slightly higher in QRPA calculations and partly because the coefficient of the isoscalar pairing interaction was readjusted after adding the two-body currents to ensure that $2 \nu \beta \beta$ matrix elements were still correctly reproduced. In any case, these studies suggest that whatever renormalization is due to many-nucleon currents will be milder in $0 \nu \beta \beta$ decay than in single- $\beta$ and $2 \nu \beta \beta$ decay.

The smaller quenching at momentum transfers near the pion mass is consistent with the studies of muon capture, where the evidence for Gamow-Teller quenching is weaker than in $\beta$ decay [232]. Exclusive charged-current electron-neutrino scattering from ${ }^{12} \mathrm{C}$ to the ground state of ${ }^{12} \mathrm{~N}$ also shows little evidence of quenching, at momentum transfer even smaller than the pion mass [233-
235]. The inclusive cross-section, for which additional multipoles are relevant, is harder to calculate and shell model calculations disagree with one another by a factor of about two [233-235]. Improved calculations, using $a b$ initio methods such as those initiated in Ref. [236, 237], are clearly needed. Further measurements, preferably in targets that complement ${ }^{12} \mathrm{C}$, would also provide valuable information about quenching at non-zero momentum transfer.

Recently, Ref. [238] included two-nucleon currents in coupled-cluster calculations of single- $\beta$ decay. The authors normal-ordered the operator with respect to a Hartree-Fock reference state rather than the simpler Fermi gas of Ref. [217]. Focusing on carbon and oxygen isotopes, they found that the two-nucleon currents reduce the strength of the $\boldsymbol{\sigma} \boldsymbol{\tau}$ operator by about $10 \%$. Though this result suggests a very small quenching of $0 \nu \beta \beta$ matrix elements, the potentially coherent contribution of the nuclear core could make many-body currents more effective in the much heavier nuclei that will be used in $\beta \beta$ decay experiments.(The normal ordering approximation that makes the coherence apparent should be carefully explored, however.)

The discussion in this section should make it clear just how important it is to characterize and untangle the sources of the $g_{A}$ problem. If they lie mainly in complicated many-body effects, the $0 \nu \beta \beta$ matrix elements may be significantly too large (though that conclusion is not uniformly supported by theoretical work in perturbation theory). On the other hand, if the problem is mostly due to many-nucleon currents, then our matrix elements are probably too large by less than a factor of two.

To determine which of the contributions is more important and plumb the consequences for $0 \nu \beta \beta$ decay, we need calculations that treat many-body correlations in a comprehensive way and include many-nucleon currents consistently. It should be possible to do all this in the next five or so years, and we discuss how in the next section. We raise just one more consideration now. As suggested by the discussion of electron-scattering strengths just above, we may be able to learn about the source of $\boldsymbol{\sigma} \boldsymbol{\tau}$ quenching by studying other electroweak processes, for instance magnetic moments and transitions. The operators for these observables also need phenomenological renormalization to agree with experimental data [146148]. The missing many-body effects are expected to be similar to those in $\beta$ decay because they emerge from the same nuclear states and similar non-relativistic operators ( $\boldsymbol{\sigma}$ and $\boldsymbol{\sigma} \tau^{z}$ vs. $\boldsymbol{\sigma} \tau^{ \pm}$). The many-nucleon corrections to the current operators can be quite different, however, because the photon has no axial-vector coupling. 


\section{IMPROVING MATRIX ELEMENT CALCULATIONS IN THE NEXT FEW YEARS}

\section{A. General Ideas}

We have seen that the important $0 \nu \beta \beta$ matrix elements still carry significant theoretical uncertainty. We believe, however, that recent developments in the shell model, QRPA, GCM, and especially in ab initio nuclear structure methods and $\chi$ EFT will finally allow the community of nuclear theorists to produce more accurate matrix elements with real estimates of uncertainty. The approach to that task will have at least three prongs:

- the improvement of current methods, in particular to accommodate all the collective correlations we know to be important,

- the development and application of $a b$ initio methods to the initial and final $\beta \beta$-decay nuclei,

- a systematic assessment of theoretical uncertainty.

In what follows we address each of these in turn.

\section{B. Improving Present Models}

\section{Extending Shell Model Configuration Spaces}

The main limitation of the shell model is that it is restricted to small configuration spaces. We have already mentioned that the effects of the neglected single-particle orbitals can be estimated perturbatively [190, 191], and that calculations in two oscillator shells are now becoming feasible [114]. To add more than a few single-particle levels in a nonperturbative way, however, requires new ideas.

One way to mitigate the shell model's shortcomings is to discard many-body configurations that have little effect on the observables one is interested in. The most established approach for doing this is the Monte Carlo shell model (MCSM) [239], which uses statistical sampling to select deformed Slater determinants and then projection to restore angular-momentum symmetry. More recent work uses importance truncation [240] or the densitymatrix renormalization group [241], though not yet in a systematic way. These schemes allow configuration spaces with dimensions several orders of magnitude larger than those that can be handled with exact diagonalization. MCSM calculations now can to work with configuration spaces of dimension $10^{23}$ [242], while exact diagonalization currently requires a space of dimension $10^{11}$ or less. The MCSM number is large enough to allow the extension of shell model configuration spaces so that they include all spin-orbit partners in $\beta \beta$-decaying nuclei and all single-particle orbitals found relevant in QRPA or EDF calculations [243, 244]. The Monte Carlo approach could also facilitate $a b$ initio no-core shell model calculations [245], which are currently limited to nuclei with less than about 20 nucleons [246, 247], in isotopes closer to those used in $\beta \beta$-decay experiments. Calculations in light nuclei are useful for other reasons as well, and we discuss them further in Sec. V C.

Extended shell-model spaces will require suitable nuclear interactions. The phenomenological modification of $H_{\text {eff }}$ that accurate calculations usually require makes it difficult to obtain reliable effective interactions in larger configuration spaces; the number of two-body matrix elements to be constrained phenomenologically increases with the size of the space. The situation would be better if we could dispense with the adjustment, the need for which may be due to the absence of three-nucleon forces in the original interaction [248]. Recent promising work [249-253] avoids any phenomenological tuning by using $\chi$ EFT two- and three-body interactions as a starting point to generate $H_{\text {eff }}$. These $a b$ initio interactions allow a better assessment of uncertainty and appear to be usable in larger configuration spaces. One can obtain transition operators the same way. We discuss the use of $\chi$ EFT and nonperturbative many-body methods to create shell model interactions and operators in Sec. V C 2.

\section{Adding Correlations to the EDF and the IBM}

The EDF matrix elements, as we noted in Sec. III C, are almost universally larger than matrix elements calculated in any other approach. The reason, as discussed in Refs. [175, 176], is missing correlations, particularly isoscalar pairing correlations. It is actually not hard to add proton-neutron pairing to the GCM; Ref. [177] did precisely that within a two-shell calculation that used a multi-separable interaction. There is no reason that the same physics cannot be added to EDF-based GCM. The main ingredient is a set of HFB calculations that allow proton-neutron mixing, i.e. quasiparticles that are a combination of a neutron and a proton as well as a particle and a hole. Computer codes to do these calculations are nearly complete [254]. Problems with the EDF-based GGM, as noted earlier, arise only when one tries to superpose the projected HFB quasiparticle vacua. Here, with a few limited exceptions, one needs a Hamiltonian rather than a functional. That does not mean, however, that EDF theory can play no role. One might, for example, use the EDF-based HFB, with proton-neutron pairing, simply to generate the projected HFB vacua. These states can be constructed so that they include all possible collective correlations, and in particular all correlations relevant to $\beta \beta$ decay. One then can use them to form a basis in which to diagonalize a real Hamiltonian such as that produced by $\chi \mathrm{EFT}$ rather than the original density functional. Functionals were designed to work well in mean-field calculations and thus should produce a good correlated basis with contributions from many more single-particle states than are in the shell 


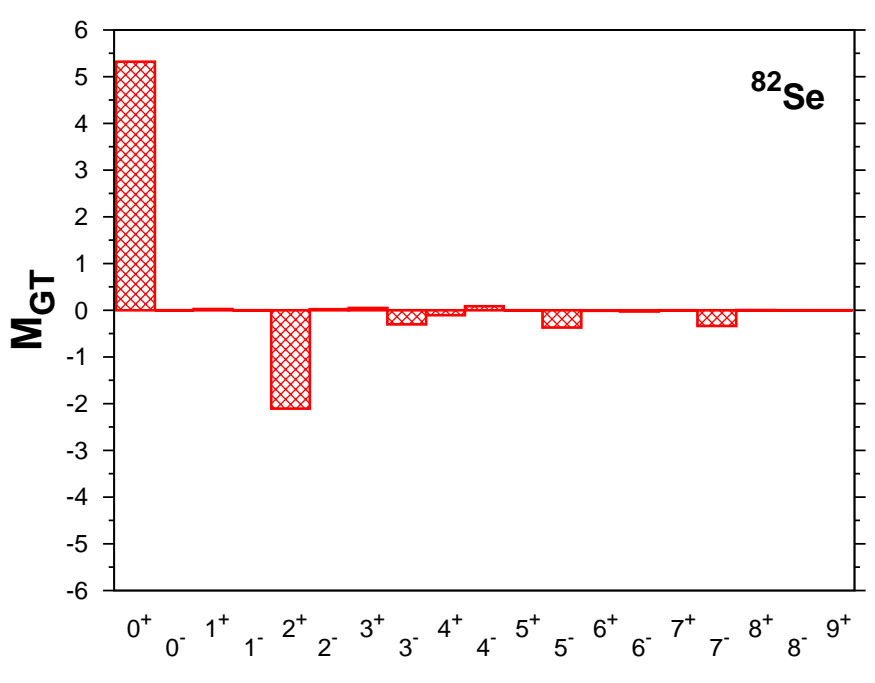

FIG. 13. Contributions of the decay of nucleon-nucleon (or proton-proton) pairs with given angular momentum and parity to the Gamow-Teller $0 \nu \beta \beta$ decay matrix element $M_{G T}$ for the ${ }^{82}$ Se transition. Figure taken from Ref. [133]

model. The Hamiltonian will then determine how these states are mixed. The result should combine many of the virtues of shell-model calculations with those of current EDF and QRPA calculations.

Even so, some correlations, e.g. those from high relative momentum, may still be absent from EDF-based approaches. Within the shell model, as we discuss in Sec. V C 2, one can construct an effective Hamiltonian and a consistent $0 \nu \beta \beta$ decay operator that captures such effects. It has not been as clear how to do that with EDF or QRPA-based calculations, in which the full many-body Hilbert space does not cleanly separate into included and neglected pieces. Recently, however, techniques to add missing correlations to approximate state vectors such as those produced by the GCM have nonetheless been developed; we discuss them in Sec. V C 3.

Finally, the IBM-2 $0 \nu \beta \beta$ decay calculations done so far, like those in EDF theory, have no explicit protonneutron pair degrees of freedom. Bosons that represent isoscalar pairs can certainly be added - a boson model with both isovector and isoscalar proton-neutron pairs is known as IBM-4 - and work is in progress to see the effects on $\beta \beta$ decay [255]. While it is not obvious that these effects will be large, the new pairs may bring the IBM matrix elements closer to those of the shell model. The IBM-2, however, already includes bosons that represent like-particle monopole and quadrupole pairs. The coupling of two spin-1 isoscalar pairs to total angular momentum zero can be represented as a combination of two like-particle $J=0$ or $J=2$ pairs, and these are exactly the degrees of freedom the usual IBM-2 bosons represent. It may be, then, that present IBM results capture most of the effects of isoscalar pairing without explicit isoscalar bosons. On the other hand, some facts indicate that isoscalar pairing effects are still missing. Figure 13, which is typical of shell model results, shows that almost the entire matrix element arises from the decay of likeparticle $J=0$ pairs or $J=2$ pairs, with a sizable cancellation between these two contributions. In the IBM-2, by contrast, the $d$-boson contribution is much smaller than that of the $s$ boson, so that there is very little cancellation [182]. The dominance of $J=0$ pairs is much like what one obtains in the shell model when isoscalar pairing is omitted [176].

\section{Higher QRPA and the Overlap Problem}

The QRPA can also be improved. It is currently limited by the simplicity of its correlations, which can be represented as one-boson states; the bosons are simplified versions of correlated quasiparticle pairs. Without many-boson states, the Pauli principle is violated and the transition strength to intermediate states overly concentrated. Accuracy requires that the many-boson states be included.

Many studies advance one scheme or another for doing that. The second QRPA and the quasiparticle timeblocking approximation (QTBA) are two representative examples. The second QRPA systematically adds all four-quasiparticle excitations to the two-quasiparticle excitations that enter the usual QRPA. Outside of preliminary investigations in Refs. [256, 257], however, it has not been systematically developed. Second RPA, without quasiparticles, is further along, both in an $a b$ inito form [258] and in conjunction with EDF theory [259, 260]. Though these schemes are computationally intensive, their quasiparticle versions should not be completely intractable and may be both accurate and flexible enough to allow ab initio calculations.

The QTBA is a Green's-function approach to linear response that supplements the iterated ring and ladder diagrams that are equivalent to the QRPA with a subset of diagrams that contain the emission and re-absorption of QRPA phonons by quasiparticles and the exchange of phonons between quasiparticles [261]. When used in conjunction with a relativistic EDF, the QTBA appears to significantly improve predictions of single- $\beta$ decay strength functions [262]. This method has the potential to significantly reduce the important physics omitted by the QRPA.

Any RPA-like method designed to calculate linear response faces a problem when applied to $\beta \beta$ decay, however. As mentioned in III B, $\beta \beta$ matrix elements involve separate transition matrix elements to each intermediatenucleus state from the initial and the final ground states. These two contributions must be multiplied and then summed over all intermediate states. But the two QRPA ground states are unrelated, so there is no way to match intermediate states produced by the excitation of one nucleus with those from the excitation of the other. 
Intermediate-state energies are different in the two cases, and the QRPA provides only transition densities, not full wave functions. Thus even attempts to compute the overlap of two different sets of intermediate-state wave functions must rely on prescriptions.

The only way to solve this problem, at least partially, is to extend the QRPA so that it does provide wave functions. One solution is to use wave functions from the simpler quasi-Tamm Dancoff approximation. Another track, taken by Refs. [263] and [264], is to represent the ground states of the initial and final nuclei by quasiparticle coupled-cluster wave functions of the schematic form

$$
|\Psi\rangle \propto \exp \sum_{\nu, a b c d}\left(\left[Y_{a b}^{\nu} / X_{c d}^{\nu}\right] \alpha_{a}^{\dagger} \alpha_{b}^{\dagger} \alpha_{c}^{\dagger} \alpha_{d}^{\dagger}\right),
$$

where the $X^{\nu}$ 's and $Y^{\nu}$ 's are the usual QRPA amplitudes for the $\nu^{\text {th }}$ excited state. This form is a natural extension of the quasi-boson wave function that the QRPA does provide when pairs of fermionic operators are replaced by bosonic operators (with respect to a particular vacuum). The quality of the approximate wave function in Eq. (21) is not yet known, however. In any event, every extension of the QRPA must face this issue. The method is a smalloscillations approximation, and the initial and final nuclei in $\beta \beta$ decay are often quite different in structure, so they cannot both be only a little different from states in the intermediate nucleus.

\section{Ab Initio Approaches}

\section{Kinds of Ab Initio Calculations}

The term $a b$ initio, literally "from the beginning," is a little vague in the nuclear-physics context. A truly firstprinciples calculation of nuclei involves solving the underlying theory, quantum chromodynamics (QCD), with quarks and gluon degrees of freedom. The ambitious framework for doing so is lattice QCD [265-267]. In spite of rapid progress, only the lightest nuclei with two, three and four nucleons have been studied thus far, and even these are treated with significant approximations, e.g. at larger-than-realistic values for the pion mass. We will not see QCD calculations of the structure of medium-mass and heavy nuclei for some time. Nevertheless, lattice QCD is valuable for the $0 \nu \beta \beta$ decay program. It can connect the parameters in the $\chi \mathrm{EFT}$ diagrams discussed in Sec. II B 2 with the underlying fundamental beyond-standard-model physics, for example [95]. More generally, it provides a way of determining all $\chi$ EFT parameters, which are often poorly constrained by data. Though doing so is computationally demanding and computed parameters are not accurate enough at present [265, 266, 268], researchers are working to improve their calculations.

In nuclear structure the term $a b$ initio usually refers to calculations that 1) take nucleons - all of them — as the degrees of freedom, and 2) use nuclear interactions and currents obtained from fits to nucleon-nucleon scattering data and properties of the lightest nuclei: the deuteron and isotopes of hydrogen and helium with 3 or 4 nucleons, and as few (slightly) heavier nuclei as possible. These fits can proceed through a meson-exchange phenomenology that describes the elastic channel of nucleon-nucleon scattering up to and perhaps beyond the pion-production threshold, leading, e.g., to the "Argonne" potentials and other similar interactions [269-271]. Phenomenological potentials of this sort work quite well in light nuclei but seem to need improvement for use in medium-mass nuclei [272].

The fits that determine nuclear interactions can also be based on $\chi$ EFT $[19-21,28]$, discussed already in connection with heavy-particle exchange (Sec. IIB 2) and many-nucleon currents (Sec. IV B). Here we give a very brief description of its use in ab initio nuclear structure. $\chi \mathrm{EFT}$ is an effective theory, based on the symmetries of QCD, that provides a perturbative framework for interactions and currents. It yields a systematic expansion of two- and many-nucleon forces and consistent one-, two-, and many-nucleon currents in powers of the parameters $p / \Lambda_{b}$ and $m_{\pi} / \Lambda_{b}$, where $p$ is a typical nucleon momentum and $\Lambda_{b}$ is the chiral-symmetry breaking scale defined in Sec. II B 2. Once the interactions are fixed, an accurate many-body method is used to calculate nuclear binding energies, radii, excitation spectra, electromagnetic transitions, decay rates, and other observables, with error estimates inferred from the power counting and tests of the many-body method.

$\chi \mathrm{EFT}$ is not without its problems. Though they sometimes produce excellent results [24], the most widely-used $\chi \mathrm{EFT}$ nuclear interactions frequently predict binding energies that are too large and radii that are too small, especially in heavy systems [26, 27, 273]. The overbinding can be partly cured by fitting the $\chi \mathrm{EFT}$ couplings to properties of nuclei as heavy as oxygen [274], even while omitting interactions beyond the three-nucleon level (and thus limiting the ability to estimate uncertainties). But the theory may still work without that step; some interactions that are obtained without it predict saturation properties [275] and agree better with experimental radii than others [276]. And fits that include consistent twoand three-nucleon forces to fourth-order in the chiral expansion are now underway [277]. Reference [278] recently presented the initial calculations in neutron matter with these interactions.

Ab initio calculations are not yet successful everywhere in the isotopic chart, but progress has been impressive. Green function Monte Carlo (GFMC) [23] and the nocore shell model (NCSM) [246, 247] have extended the scope of $a b$ initio studies light nuclei to detailed spectroscopy, reactions, and nuclear response. Ab initio work on heavy nuclei has a fairly long history; milestones include coupled-clusters (CC) calculations in ${ }^{16} \mathrm{O}$ and ${ }^{40} \mathrm{Ca}$ [279-281], the introduction of the now essential normalordering procedure for treating three-body interactions 
[282] and the use of $\chi$ EFT two- and three-nucleon interactions together with many-body perturbation theory to derive a shell-model effective interaction for oxygen isotopes [249]. The same ideas were later used in heavier nuclei [24]. Non-perturbative $a b$ initio methods, including CC theory [25], the in-medium similarity renormalization group (IMSRG) [26], the self-consistent Green's function method [27], and nuclear lattice simulations [28, 283] have developed quickly as computers have become more powerful. We describe some of them here.

The application of GFMC [23] to nuclear structure was a true turning point for the field [284]. The method starts with an approximate wave function that includes two and three-body correlations in all spin and isospin channels and is usually obtained through a variational Monte Carlo calculation. This wave function is then evolved in imaginary time to filter out spurious components. The procedure, which exploits the locality of Argonne twobody and "Illinois" three-body interactions, reproduces binding energies with an r.m.s. error of $0.36 \mathrm{MeV}$ and the energies of the lowest excited states with an r.m.s. error of $0.5 \mathrm{MeV}$ in nuclei up to ${ }^{12} \mathrm{C}$ [23]. The approach also works with local versions of $\chi$ EFT interactions [285] and is valuable for the computation of electromagnetic and weak response [23, 228, 237]. Unfortunately, computation time scales exponentially with the number of particles, and the method cannot soon be extended to the heavy nuclei in $\beta \beta$ decay experiments. Although other forms of quantum Monte Carlo, e.g. auxiliary diffusion Monte Carlo, should reach heavier systems [272], a treatment of open shell nuclei appears to lie at least a few years in the future. The promise of quantum Monte Carlo, however, is exceptional. And the GFMC can already be used in light nuclei to help diagnose the source of the $g_{A}$ quenching we discussed in Sec. IV.

The NCSM [246, 247], already discussed briefly in Sec. $\mathrm{VB} 1$, is a shell model in which none of the $A$ nucleons are forced to occupy particular single-particle orbitals, and in which one can explicitly check the convergence of results as the configuration space grows. The space is usually truncated so as to keep all configurations with total oscillator excitation energy $\mathcal{N} \hbar \omega$ or below (for some $\mathcal{N})$ compared to the lowest-energy configuration. The parameter $\mathcal{N}$ ranges from hundreds for the deuteron to just a few for nuclei with a valence $s d$ shell. Whether any particular $\mathcal{N}$ is sufficient depends on the nuclear interaction employed. For that reason, it is helpful to "evolve" or "soften" the interaction via the SRG, so that the calculation converges in a smaller configuration space (with the drawback that the evolution generates many-body interactions). The initial unevolved interaction can come from a phenomenological model or from $\chi \mathrm{EFT}$.

In general, the NCSM produces energies that are comparable in accuracy to those of the GFMC, but can be extended to somewhat heavier nuclei by using importance truncation to exclude irrelevant configurations [286]. Unfortunately, as with the GFMC, computation time scales exponentially with the number of particles and an ex- tension to the medium mass $\beta \beta$-decaying nuclei will be difficult. NCSM calculations will still be useful in $\beta \beta$ decay research, however. In addition to investigating $g_{A}$ in single- $\beta$ decay [287], they allow tests of schemes to generate effective operators for the usual shell model (with a core) [60].

The $a b$ initio methods that can soon be applied in heavier nuclei generally have the benefit of being explicitly "size extensive". That term refers to the correct (roughly linear) scaling of binding energy with the mass number $A$ at any level of truncation. The computation time for these methods, which include CC, the IMSRG, and the self-consistent Green's function approach, is a polynomial in $A$ rather than an exponential. A basic CC calculation, for example, takes a time that is proportional to $A^{4}$.

The starting point for CC theory [25] is an exponential ansatz for the ground state $\left|\Psi_{0}\right\rangle$ of a closed-shell eveneven nucleus:

$$
\left|\Psi_{0}\right\rangle=e^{T}\left|\varphi_{0}\right\rangle
$$

where $|\varphi\rangle$ is a Slater determinant and

$$
T=\frac{1}{4} \sum_{i, a} t_{i}^{a} a_{a}^{\dagger} a_{i}+\frac{1}{36} \sum_{i j, a b} t_{i j}^{a b} a_{a}^{\dagger} a_{b}^{\dagger} a_{i} a_{j}+\ldots
$$

with the indices $a, b$ denoting particle orbitals (above the Fermi surface) and $i, j$ hole orbitals (below the Fermi surface). The $t$ 's are amplitudes determined by projecting the Schrödinger equation onto one-particle one-hole excitations (generating an equation for the $t_{i}^{a}$ ), two-particle two-hole excitations (generating one for the $T_{i j}^{a b}$ ), etc. If the operator $T$ is truncated at that point, the method is called CC-SD, where SD stands for "singles and doubles," and if it is continued to the next order it is called CC-SDT, where T stands for "triples." Still higher-order terms, involving four-particle clusters, are rarely considered. CC theory has been applied to closed-shell nuclei as heavy as ${ }^{132} \mathrm{Sn}[273]$ and to compute not only energies but also charge and matter radii, single- $\beta$ decay rates, etc. $[238,276,288]$.

To calculate $\beta \beta$ decay elements in a closed shell nucleus such as ${ }^{48} \mathrm{Ca}$, one needs, in addition to the ground-state $\left|\Psi_{0}\right\rangle$ of that nucleus, the ground state $\left|\Psi_{0}^{\prime}\right\rangle$ of the final nucleus ${ }^{48} \mathrm{Ti}$, which has both valence protons and neutrons. There one uses the "equations-of-motion" method [25]. The ground state of the final nucleus is represented as $\left|\Psi_{0}^{\prime}\right\rangle=\mathcal{R}\left|\Psi_{0}\right\rangle$, where

$$
\mathcal{R}=\sum_{i j a b} r_{i j}^{a b} p_{a}^{\dagger} p_{b}^{\dagger} n_{i} n_{j}+\frac{1}{6} \sum_{i j k a b c} r_{i j k}^{a b c} p_{a}^{\dagger} p_{b}^{\dagger} N_{c}^{\dagger} N_{k} n_{i} n_{j}+\ldots
$$

The operator $\mathcal{R}$ creates two-particle two-hole excitations, three-particle three-hole, excitations, etc. of the $\mathrm{CC}{ }^{48} \mathrm{Ca}$ ground state. Equations for the corresponding $r$ amplitudes, like those for the $t$ amplitudes, follow from projection of the Schrödinger equation. The application of 
$\mathrm{CC}$ theory in mid-shell nuclei requires a more elaborate construct, which we discuss in Sec. V C 2.

The IMSRG [26] is a set of flow equations for a unitary transformation that in the asymptotic limit decouples some pre-selected subspace of states from the rest. In its simplest version, the flow equations for the Hamiltonian take the form

$$
\frac{d}{d s} H(s)=[\eta(s), H(s)], \quad H(\infty)=H_{\mathrm{eff}}
$$

with $s$ the flow parameter and the "generator" $\eta(s)$ given by

$$
\eta(s)=\left[H_{d}(s), H_{o d}(s)\right]
$$

Here, $H_{d}$, (where $d$ stands for diagonal, though its meaning can be more general) is the part of the Hamiltonian that does not couple the space one is interested in to the rest, and $H_{o d}$ is the part that does. It is not hard to show that as the flow progresses, $H_{o d}$ is driven to zero. In a closed-shell nucleus, if one chooses to decouple a reasonable zeroth-order approximation to the ground state e.g. a Hartree-Fock Slater determinant - one ends up with a Hamiltonian $H(s=\infty)$ for which that approximation is the exact ground state. Because the evolution equation is unitary, the eigenvalue is the ground-state energy. A consistent flow equation governs the evolution of transition operators other than the Hamiltonian.

Application of the method in practice is more complicated than this simple description indicates, in a number of ways. First, the flow equations generate threebody interactions, four-body interactions, etc., (as in any SRG flow) even if the initial Hamiltonian has only oneand two-body terms. In practice, the equations are usually truncated to include only normal-ordered one- and two-body pieces. But the normal-ordering, carried out with respect to the initial Slater determinant in the example above, incorporates the most important effects of higher-body terms, just as it includes the most important pieces of the two-body interaction in a Hartree-Fock single-particle Hamiltonian. Second, the flow equations above are stiff and solutions hard to obtain. Other forms for the generator $\eta$ are better behaved and still drive $H_{o d}$ to zero. Also, the evolution of transition operators is easiest with a single set of equations for the unitary transformation that does the decoupling rather than a separate set for every operator [289]. Finally, in openshell systems a Slater determinant is not a good zerothorder approximation to the exact ground state and the method fails.

The approach described here has been used to calculate energies, charge radii, and matter radii in nuclei that range from the lightest to nickel isotopes [26, 290]. In Secs. V C 2 and VC 3 we describe ways the method can be extended.

\section{Effective Operators for the Shell Model from Ab Initio Calculations}

The computation times for CC theory and the IMSRG both scale in a polynomial way with $A$, but both methods need to be modified in open-shell nuclei like those that are used in $\beta \beta$ decay experiments. One approach to these nuclei is to use the methods indirectly, to construct effective interactions and operators for use in shell-model configuration spaces. Then one can simply diagonalize the effective interaction $H_{\text {eff }}$ and compute the transition matrix element of the effective $\beta \beta$ decay operator in exactly the same way as with the usual (phenomenological) shell model.

One of the oldest procedures for constructing effective operators, and the one that is used in conjunction with CC theory, employs what is known as an Okubo-LeeSuzuki mapping [291-293]. The idea is to do ab initio calculations in the closed-shell nucleus - the shellmodel "core" — in the nucleus with one nucleon outside the core, and in the nucleus with two nucleons outside the core [294]. (The procedure can be continued, though it gets increasingly complicated). CC theory uses the equations-of-motion method in the nuclei with one or two nucleons outside the core. With the lowest few states in these nuclei computed, one obtains the energy of the shell-model core from the ground-state energy in the CC closed-shell calculation, and the shellmodel single-particle energies from the lowest CC energies in the nuclei with one additional nucleon. To get the two-body matrix elements that define an effective interaction $H_{\text {eff }}$ in the dimension- $d$ shell-model configuration space, one projects $d \mathrm{CC}$ eigenstates $|k\rangle$ in the nuclei with two particles outside the core onto the shell-model space, and then orthogonalizes the projected states to get the shell-model states $|\tilde{k}\rangle$. The orthogonalization procedure, sometimes called "symmetric orthogonalization" minimizes the quantity

$$
\sum_{k=1}^{d}(\langle k|-\langle\tilde{k}|)(|k\rangle-|\tilde{k}\rangle)
$$

so that the shell-model states are, on average, as close as possible to the original $\mathrm{CC}$ sates. With the mapping specified, one simply sets the matrix elements of all twobody effective operators between the shell model $|\tilde{k}\rangle$ 's to equal those of the "bare" operators between the CC $|k\rangle$ 's. The program has been carried out in nuclei with a valence $s d$ shell $[250,295]$.

In the IMSRG, the procedure for obtaining effective shell-model operators is a little different [26]. The idea is to construct the generator $\eta$ in Eq. (25) so that the Hamiltonian does not connect states in which the core is filled and the "active" particles are all in the shellmodel configuration space to the rest of the Hilbert space. The resulting Hamiltonian $H(s=\infty)$ is the effective shell-model interaction $H_{\text {eff }}$. To capture residual threenucleon interactions among active particles (generated 


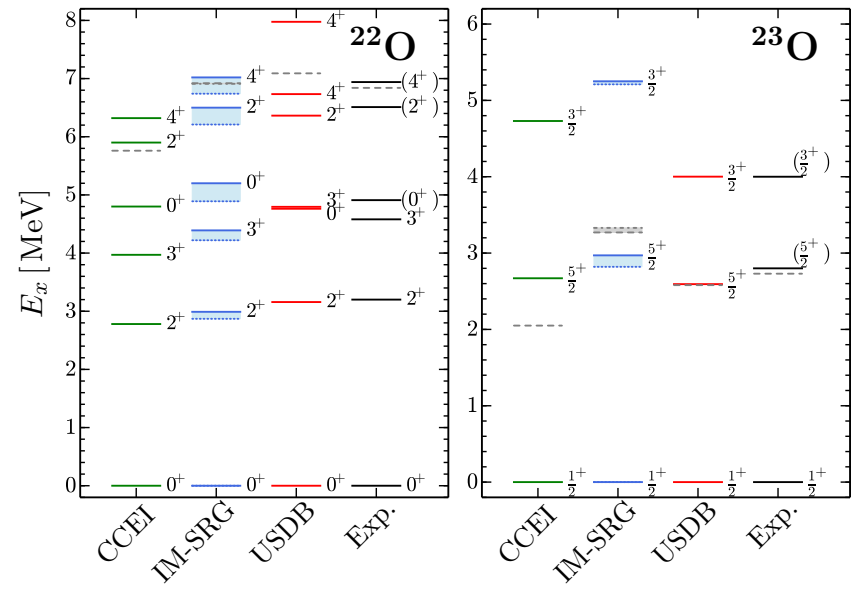

FIG. 14. Excitation spectra of two neutron-rich oxygen isotopes, with effective interactions obtained in coupled-clusters theory (CCEI) and the IMSRG (IM-SRG), compared with spectra produced by a good phenomenological shell-model interaction (USDB [298]), and with experiment. The bands in the IM-SRG results indicate the uncertainty associated with the choice of frequency for the harmonic oscillator potential that generates basis states. Figure taken from Ref. [299].

by the SRG flow) an ensemble "reference state," yielding partially occupied orbitals, is used for the normalordering [296]. Since the ensemble depends on the nucleus being studied, a different $H_{\text {eff }}$ results for each isotope. As with the simpler version of the IMSRG discussed above, one obtains the effective $0 \nu \beta \beta$ decay operator by applying the same unitary transformation that generates $H_{\text {eff }}$ to the bare $0 \nu \beta \beta$ decay operator. IMSRG calculations of this sort have shown the ability to compute binding energies and spectra in nuclei with valence $s d$ - and $p f$-shell nucleons [251, 296, 297].

The $a b$ initio shell model program is still in its early stages, both in CC theory and the IMSRG, but has already generated promising results. Figure 14 shows spectra computed by the shell-model effective interactions that result from both procedures. The spectra are not exact for a number of reasons: 1) High-order pieces of the $\chi$ EFT Hamiltonian are neglected, 2) the CC expansion and the IMSRG flow equation are truncated, 3) the Okubo-Lee-Suzuki mapping is carried out only up to two valence particles (in CC theory). Nevertheless, the results are comparable to those obtained with a standard phenomenological shell-model interaction, which was fit to spectroscopic data in the same mass region. The $a b$ initio approaches have clear promise.

The computation of matrix elements for other operators is just beginning, and it remains to be seen how successful it will be. There is no reason in principle, however, that matrix elements of single- $\beta$ and $\beta \beta$ decay operators should be much less accurate than those of the effective interaction. The framework offers an opportunity to make the shell model a much more rigorous tool for $\beta \beta$ decay calculations. Researchers will pursue it vigorously.

\section{Multi-reference IMSRG}

To the extent that the closure approximation is good (see Sec. II B 1), only ground states in the initial and final nuclei are required to calculate the $0 \nu \beta \beta$ decay transition rate. The effective-operator methods for use in the shellmodel, just described in Sec. V C 2, are quite general and produce information not only about the ground state but also about a whole set of low-lying states. An approach that restricts itself to ground states might be more efficient. Both the CC and IMSRG procedures, as described in Sec. V C 1, begin with a Slater determinant as an approximate ground state, a reference state to which they add correlations. With the exception of ${ }^{48} \mathrm{Ca}$, however, none of the $\beta \beta$ emitters of potential use can be described even approximately by a Slater determinant.

Fortunately, both the CC and IMSRG approaches can be extended to more general reference states. The extension is perhaps easier in the IMSRG, which relies on the reference only for the normal ordering of many-body operators that includes their most important effects within zero-, one-, and two-body operators. And normal ordering has, in fact, been generalized to arbitrary reference states, most prominently in Refs. [300] and [301]. The generalized normal ordering has most of the benefits of the usual normal ordering, including an associated (generalized) Wick's theorem that expresses the product of an $\mathcal{N}$-body normal-ordered operator with an $\mathcal{M}$ body normal-ordered operator as a linear combination of normal-ordered operators of rank less than or equal to $\mathcal{N}+\mathcal{M}$. The only drawback is that the contractions that enable this procedure generate up to $(\mathcal{N}+\mathcal{M})$-body density matrices, rather than just the one-body density matrices produced by the usual Wick's theorem. But that is a small price for the benefit: The generalized normal ordering allows one to include most of the effects of three- and higher-body operators generated by the flow equations by discarding just the normal-ordered pieces of these operators, making the equations as tractable as in the ordinary IMSRG [299]. The use of a generalized reference state in this way is usually called the multi-reference IMSRG.

Thus far, the method has been applied only to spherical nuclei (e.g. calcium and nickel isotopes) with reference states obtained from spherical HFB calculations [26], but there is no reason it cannot be applied more generally, with a shell-model reference state or one from an EDF. That would allow important collective correlations to be included directly in the reference, with the flow equations adding the most important parts of other less identifiable correlations. Work in this direction is in progress [299]. 


\section{Assessing Error}

At present, nothing resembling a solid quantitative measure of uncertainty in the $0 \nu \beta \beta$ matrix element exists. In Fig. 5 the error bars on some of the QRPA values represent only the spread in results coming from the use of two different nucleon-nucleon interactions, a crude sort of statistical uncertainty. The bars on some of the shellmodel values come from the use of two prescriptions for including short-range correlations and represent a crude estimate of a part of what we will call the systematic uncertainty associated with the model.

With the lack of real uncertainty estimates, experimentalists usually rely on the range of numbers obtained by seemingly reliable models (e.g. the one spanned in Fig. 5). The problems with that approach to uncertainty are obvious: on the one hand the range is large and may include the results of poor calculations and, on the other, all the models may be omitting the same important physics (such as that associated with the $g_{A}$ problem discussed in Sec. IV). The point we have made in this review is that models must incorporate all known correlations relevant for $0 \nu \beta \beta$ decay and reproduce related observables. Capturing the important correlations is easiest in an ab initio calculation that systematically controls error at each step, even if that control is imperfect. Phenomenological models can be extended to include more correlations in the ways discussed in Sec. V B, and can be benchmarked against $a b$ initio efforts. In this way all the many-body methods we have discussed can be useful in improving the accuracy of matrix elements and estimating their uncertainty.

The absence of error quantification in $0 \nu \beta \beta$ decay is not an exception. Because it has been traditionally been dominated by phenomenological approaches, nuclear-structure theory has traditionally neglected even to try to estimate uncertainty. This situation is slowly changing, however, and ab initio methods and $\chi \mathrm{EFT}$ forces provide natural tools to quantify theoretical error. References [302-304] have taken the first steps in quantifying error, and Ref. [305] provides initial estimates of theoretical uncertainty in $s d$-shell nuclei. We can expect this sort of work to progress rapidly.

Dividing error estimates into two kinds - statistical and systematic - is useful. Statistical error is probably easier to assess. We discuss both below, in turn.

\section{Statistical Error}

By statistical error we mean, roughly, the uncertainty due to the choice of parameters within a given model or method. The matrix elements of the effective interaction $H_{\text {eff }}$ for the shell model, the value of the parameter $g_{p p}$ in the QRPA, the effective value of the spinisospin coupling $g_{A}$ in all phenomenological models, and the low-energy constants that specify the $\chi \mathrm{EFT}$ forces and currents in $a b$ initio calculations are all examples of model parameters. No matter what statistical protocols are used, the statistical uncertainty in $0 \nu \beta \beta$ matrix elements will emerge from an analysis of the correlation between them and other observables for which we have data. Rates of single- $\beta$ decay and $2 \nu \beta \beta$ decay will be strongly correlated. Gamow-Teller transition strengths, excitation spectra, occupation numbers, and two-neutron transfer probabilities [306, 307] can also be correlated. These observables will constrain parameters and, in turn, the predictions for $0 \nu \beta \beta$ matrix elements.

Some analysis of this kind has been carried out in the QRPA [53,115]; the $0 \nu \beta \beta$ matrix elements tend to vary by about one unit when parameters are changed. Reference [308] examined the correlations among matrix elements in different isotopes. References $[54,193]$ examined the sensitivity of matrix elements to $H_{\text {eff }}$ in the shell model. Again, as can be seen in Fig. 5, results vary by about one unit. This amount is similar to the average difference between matrix elements calculated with nonrelativistic and relativistic EDFs. (Only in ${ }^{150} \mathrm{Nd}$ is the difference much larger.) All these results suggest that systematic uncertainty is larger than statistical uncertainty.

The statistical uncertainty related to $g_{A}$ has been explored via the correlation of this parameter with rates of electron capture, single- $\beta$ decay, and $2 \nu \beta \beta$ decay data, first for selected isotopes [309] and more recently in a more complete study for a wide range of nuclei [310]. References $[217,218,231]$ studied the sensitivity of $g_{A}$ to variations of the couplings in the $\chi \mathrm{EFT}$ two-body currents. More comprehensive work in the same vein is important. We need to examine sensitivity in other computational approaches and correlations with other observables in the same way.

\section{Systematic Error and $g_{A}$}

Systematic error, the uncertainty related to the insufficiency of models, is harder to assess. Once a model incorporates as many of the known-to-be-important correlations as possible - isoscalar pairing, or like-particle pairing correlations involving single-particle orbitals away from the Fermi level, for example - its deficiencies can only be assessed by a confrontation with data and with other models. Here there is a lot to do. The statistical error analysis just discussed can shed light on systematic error as well; if there are no sensible sets of parameters that lead to an accurate description of the available data, we know that our model is systematically deficient [311].

Benchmarking is another important tool. The $a b i n i$ tio methods reviewed here unavoidably entail some kind of truncation, but a different kind in each. For instance, $\mathrm{CC}$ theory and the IMSRG both impose a cutoff on the maximum numbers of particles within a cluster (roughly speaking) or the maximum number of single-particle oscillator orbitals. Meanwhile, the NCSM instead allows only configurations with excitation energy below a cer- 
tain cutoff and the GFMC discretizes time and constrains paths to cope with the fermion sign problem. Comparing the predictions of these approaches in nuclei for which they are all implementable - e.g. nuclei with a valence $s d$ shell - can reveal a lot. Agreement between ground state energies of oxygen isotopes in this kind of intermethod test is at the few percent level [24]. There is no reason not to test calculations of $0 \nu \beta \beta$ matrix elements in the same way, even though the corresponding transitions are not observable. Benchmarking complementary methods can reveal the importance of each kind of truncation, and agreement among methods could reduce uncertainty considerably.

The renormalization of the spin-isospin operator $\left(g_{A}\right.$ quenching) is particularly ripe for analysis in light nuclei. The $a b$ initio methods appear powerful enough to include all important correlations as well as the leading two-body currents in those isotopes, for which there is no shortage of measured single- $\beta$ decay rates. The analysis can also include magnetic moments and transitions, which, like $\beta$-decay, involve the spin operator and sometimes require that its strength be quenched to agree with data. If $a b$ initio calculations all fail to reproduce experimental $\beta$-decay rates, then there will have to be some other source of systematic uncertainty that we cannot currently imagine. If, on the other hand, one or more succeed, the result will be a reduction of systematic uncertainty in the $0 \nu \beta \beta$ decay matrix elements and a clear path to ab initio $\beta \beta$ decay calculations in the heavier nuclei used or contemplated by experimentalists. Understanding the cause of quenching will also allow the development of realistic procedures for improving the more phenomenological methods.

These tests and similar ones in heavier nuclei, where data for $2 \nu \beta \beta$ decay can be directly confronted, will not in themselves yield a precise error bar for $0 \nu \beta \beta$ matrix elements, especially for those in ${ }^{76} \mathrm{Ge}$ or ${ }^{136} \mathrm{Xe}$. One can never be sure that all sources of systematic error are understood. But a good protocol (e.g. based on popular Bayesian methods) should lead to error bars in which we can be reasonably confident, much more confident than we are in the "uncertainty" associated with the spread in the predictions of only partially tested models. A good protocol will require collaboration among all kinds of theorists. The necessary groundwork for such collaboration now exists.

\section{SUMMARY AND PROSPECTS}

Next-generation experiments could well be in a position to observe $0 \nu \beta \beta$ decay if neutrinos are Majorana particles and neutrino masses are arranged according to the inverted hierarchy. They may also be able to discover new fundamental physics even if the mass hierarchy is normal. The rate of any kind of $\beta \beta$ decay, however, depends on nuclear matrix elements, and informed decisions about which and how much material to use in experiments rely on our ability to calculate them accurately. If $0 \nu \beta \beta$ decay is actually observed, the matrix elements will play a key role in identifying the mechanism responsible and/or extracting information about neutrino masses.

At present, a handful of many-body methods produce matrix elements that differ from one another by factors of up to about three. The range suggests that each approach is missing important physics. We have identified the most obvious weaknesses of each approach — usually either a restricted configuration space or simplified correlations - and have discussed programs to improve each of them. Phenomenological models are not in themselves sufficient to produce truly reliable matrix elements, however, as the longstanding need to renormalize $g_{A}$ makes clear. Without a more systematic approach to nuclear structure we are unlikely either to understand the implications of $g_{A}$ quenching for $0 \nu \beta \beta$ decay or to assign meaningful theoretical uncertainty to matrix elements.

We are hopeful that $a b$ initio nuclear-structure theory will help with these tasks. Once limited to very light nuclei, ab initio methods have progressed rapidly and will soon allow the matrix element of the lightest $\beta \beta$ emitter, ${ }^{48} \mathrm{Ca}$, to be computed. They promise to do the same in the heavier isotopes that are currently used in experiments: ${ }^{76} \mathrm{Ge},{ }^{130} \mathrm{Te}$, and ${ }^{136} \mathrm{Xe}$. Comparing the by now wide variety of $a b$ initio methods to one another in lighter nuclei will facilitate the assignment of uncertainty. The $a b$ initio methods can also revitalize traditional models, e.g. by supplying input for the shell model or using states produced by EDF calculations as references. And they provide tools that should let us, finally, solve the $g_{A}$ problem. That step alone will significantly reduce the systematic uncertainty in $0 \nu \beta \beta$ matrix elements.

We are in an exciting time. After years of development, $\beta \beta$ decay experiments are poised to scale up, opening new windows into fundamental physics. We are optimistic that nuclear theory will soon produce the accurate matrix elements and controlled theoretical error that these experiments demand.

\section{ACKNOWLEDGMENTS}

We would like to thank B. A. Brown, E. Caurier, S. R. Elliott, D. Gazit, R. Henning, N. Hinohara, J. D. Holt, M. Horoi, F. Iachello, C. F. Jiao, Y. Iwata, G. MartínezPinedo, M. T. Mustonen, F. Nowacki, T. Otsuka, A. Poves, T. R. Rodríguez, F. Šimkovic, A. Schwenk, N. Shimizu, P. Vogel, and J. M. Yao for many useful discussions on the topics we covered in this review, and $\mathrm{H}$. Hergert, T. Papenbrock, S. Pastore, T. R. Rodríguez and S. R. Stroberg for useful comments on the manuscript. J. E. acknowledges support from DOE grants DE-FG0297ER41019, DE-SC0008641, and DE-SC0004142. J. M. was supported by an International Research Fellowship from JSPS and JSPS Grant-in-Aid for Scientific Research No. 26.04323. 
[1] Reyco Henning, "Current status of neutrinoless doublebeta decay searches," Rev. Phys. 1, 29-35 (2016).

[2] Stefano Dell'Oro, Simone Marcocci, Matteo Viel, and Francesco Vissani, "Neutrinoless double beta decay: 2015 review," Adv. High Energy Phys. 2016, 2162659 (2016).

[3] O. Cremonesi and M. Pavan, "Challenges in Double Beta Decay," Adv. High Energy Phys. 2014, 951432 (2014).

[4] J. J. Gómez-Cadenas, J. Martín-Albo, M. Mezzetto, F. Monrabal, and M. Sorel, "The search for neutrinoless double beta decay," Riv. Nuovo Cim. 35, 29-98 (2012).

[5] A. Gando et al. (KamLAND-Zen Collaboration), "Search for Majorana Neutrinos near the Inverted Mass Hierarchy Region with KamLAND-Zen," Phys. Rev. Lett. 117, 082503 (2016).

[6] J. B. Albert et al. (EXO-200 Collaboration), "Search for Majorana neutrinos with the first two years of EXO-200 data," Nature 510, 229-234 (2014).

[7] J. Martín-Albo et al. (NEXT Collaboration), "Sensitivity of NEXT-100 to neutrinoless double beta decay," JHEP 05, 159 (2016).

[8] M. Agostini et al. (GERDA Collaboration), "Results on Neutrinoless Double- $\beta$ Decay of ${ }^{76} \mathrm{Ge}$ from Phase I of the GERDA Experiment," Phys. Rev. Lett. 111, 122503 (2013).

[9] N. Abgrall et al. (Majorana Collaboration), "The Majorana Demonstrator Neutrinoless Double-Beta Decay Experiment," Adv. High Energy Phys. 2014, 365432 (2014).

[10] K. Alfonso et al. (CUORE Collaboration), "Search for Neutrinoless Double-Beta Decay of ${ }^{130}$ Te with CUORE0," Phys. Rev. Lett. 115, 102502 (2015).

[11] S. Andringa et al. (SNO+ Collaboration), "Current Status and Future Prospects of the SNO+ Experiment," Adv. High Energy Phys. 2016, 6194250 (2016).

[12] S. Blot et al. (NEMO-3 and SuperNEMO Collaborations), "Investigating decay with the NEMO-3 and SuperNEMO experiments," J. Phys. Conf. Ser. 718, 062006 (2016).

[13] J. W. Beeman et al. (LUCIFER Collaboration), "Double-beta decay investigation with highly pure enriched ${ }^{82}$ Se for the LUCIFER experiment," Eur. Phys. J. C 75, 591 (2015).

[14] T. Iida et al. (CANDLES Collaboration), "Status and future prospect of ${ }^{48} \mathrm{Ca}$ double beta decay search in CANDLES," J. Phys. Conf. Ser. 718, 062026 (2016).

[15] HyangKyu Park (AMoRE Collaboration), "The AMoRE: Search for Neutrinoless Double Beta Decay in 100 Mo," Nucl. Part. Phys. Proc. 273-275, 2630-2632 (2016).

[16] J. Ebert et al. (COBRA Collaboration), "The COBRA demonstrator at the LNGS underground laboratory," Nucl. Instrum. Meth. A 807, 114-120 (2016).

[17] Neha Dokania, V. Singh, C. Ghosh, S. Mathimalar, A. Garai, S. Pal, V. Nanal, R. G. Pillay, A. Shrivastava, and K. G. Bhushan, "Radiation Background Studies for $0 \nu \beta \beta$ decay in ${ }^{124} \mathrm{Sn}, "$ arXiv:1504.05433.

[18] Yoshiyuki Fukuda, "ZICOS - New project for neutrinoless double beta decay experiment using zirconium complex in liquid scintillator," J. Phys. Conf. Ser. 718, 062019 (2016).

[19] E. Epelbaum, H.-W. Hammer, and U.-G. Meißner, "Modern theory of nuclear forces," Rev. Mod. Phys. 81, 1773 (2009).

[20] R. Machleidt and D. R. Entem, "Chiral effective field theory and nuclear forces," Phys. Rept. 503, 1-75 (2011).

[21] Hans-Werner Hammer, Andreas Nogga, and Achim Schwenk, "Three-body forces: From cold atoms to nuclei," Rev. Mod. Phys. 85, 197 (2013).

[22] R. Machleidt and F. Sammarruca, "Chiral EFT based nuclear forces: Achievements and challenges," Phys. Scr. 91, 083007 (2016).

[23] J. Carlson, S. Gandolfi, F. Pederiva, Steven C. Pieper, R. Schiavilla, K. E. Schmidt, and R. B. Wiringa, "Quantum monte carlo methods for nuclear physics," Rev. Mod. Phys. 87, 1067-1118 (2015).

[24] K. Hebeler, J. D. Holt, J. Menéndez, and A. Schwenk, "Nuclear forces and their impact on neutron-rich nuclei and neutron-rich matter," Ann. Rev. Nucl. Part. Sci. 65, 457-484 (2015).

[25] G. Hagen, T. Papenbrock, M. Hjorth-Jensen, and D. J. Dean, "Coupled-cluster computations of atomic nuclei," Rept. Prog. Phys. 77, 096302 (2014).

[26] H. Hergert, S. K. Bogner, T. D. Morris, A. Schwenk, and K. Tsukiyama, "The In-Medium Similarity Renormalization Group: A Novel Ab Initio Method for Nuclei," Phys. Rept. 621, 165-222 (2016).

[27] V. Somà, A. Cipollone, C. Barbieri, P. Navrátil, and T. Duguet, "Chiral two- and three-nucleon forces along medium-mass isotope chains," Phys. Rev. C 89, 061301 (2014).

[28] Ulf-G Meißner, "The long and winding road from chiral effective Lagrangians to nuclear structure," Phys. Scr. 91, 033005 (2016).

[29] F. T. Avignone III, Steven R. Elliott, and Jonathan Engel, "Double beta decay, Majorana neutrinos, and neutrino mass," Rev. Mod. Phys. 80, 481 (2008).

[30] J.D. Vergados, H. Ejiri, and F. Šimkovic, "Theory of neutrinoless double beta decay," Rept. Prog. Phys. 75, 106301 (2012).

[31] Y. Fukuda et al. (Super-Kamiokande Collaboration), "Evidence for oscillation of atmospheric neutrinos," Phys. Rev. Lett. 81, 1562-1567 (1998).

[32] Q. R. Ahmad et al. (SNO Collaboration), "Direct evidence for neutrino flavor transformation from neutral current interactions in the Sudbury Neutrino Observatory," Phys. Rev. Lett. 89, 011301 (2002).

[33] K. Eguchi et al. (KamLAND Collaboration), "First results from KamLAND: Evidence for reactor antineutrino disappearance," Phys. Rev. Lett. 90, 021802 (2003).

[34] K. A. Olive et al. (Particle Data Group), "Review of Particle Physics," Chin. Phys. C 38, 090001 (2014).

[35] M. C. Gonzalez-Garcia, Michele Maltoni, and Thomas Schwetz, "Updated fit to three neutrino mixing: status of leptonic CP violation," JHEP 11, 052 (2014).

[36] K. Abe et al. (T2K Collaboration), "Measurements of neutrino oscillation in appearance and disappearance channels by the $\mathrm{T} 2 \mathrm{~K}$ experiment with $6.6 \times 10^{20}$ protons 
on target," Phys. Rev. D 91, 072010 (2015).

[37] P. Adamson et al. (NOvA Collaboration), "First measurement of electron neutrino appearance in NOvA," Phys. Rev. Lett. 116, 151806 (2016).

[38] S. M. Bilenky, C. Giunti, J. A. Grifols, and E. Masso, "Absolute values of neutrino masses: Status and prospects," Phys. Rep. 379, 69 (2003).

[39] M. Doi, T. Kotani, and E. Takasugi, "Double beta decay and majorana neutrino," Prog. Theor. Phys. 83, 1 (1985).

[40] W. C. Haxton and G. J. Stephenson Jr., "Double beta decay," Prog. Part. and Nucl. Phys. 12, 409 (1984).

[41] T. Tomoda, "Double beta decay," Rep. Prog. Phys. 54, 53 (1991).

[42] M. P. Mendenhall et al. (UCNA Collaboration), "Precision measurement of the neutron $\beta$-decay asymmetry," Phys. Rev. C 87, 032501 (2013).

[43] O. Dumbrajs, R. Koch, H. Pilkuhn, G. C. Oades, H. Behrens, J. J. De Swart, and P. Kroll, "Compilation of Coupling Constants and Low-Energy Parameters," Nucl. Phys. B 216, 277-335 (1983).

[44] Veronique Bernard, Latifa Elouadrhiri, and Ulf-G. Meissner, "Axial structure of the nucleon," J. Phys. G: Nucl. Part. Phys. 28, R1-R35 (2002).

[45] K. Muto, "Neutrinoless double beta decay beyond closure approximation," Nucl. Phys. A 577, 415C-420C (1994).

[46] Fedor Šimkovic, Rastislav Hodak, Amand Faessler, and Petr Vogel, "Relation between the $0 \nu \beta \beta$ and $2 \nu \beta \beta$ nuclear matrix elements revisited," Phys. Rev. C 83, 015502 (2011).

[47] R. A. Sen'kov and M. Horoi, "Neutrinoless double- $\beta$ decay of ${ }^{48} \mathrm{Ca}$ in the shell model: Closure versus nonclosure approximation," Phys. Rev. C 88, 064312 (2013).

[48] R. A. Sen'kov, M. Horoi, and B. A. Brown, "Neutrinoless double- $\beta$ decay of ${ }^{82} \mathrm{Se}$ in the shell model: beyond closure approximation," Phys. Rev. C 89, 054304 (2014).

[49] R. A. Sen'kov and M. Horoi, "Accurate shell-model nuclear matrix elements for neutrinoless double- $\beta$ decay," Phys. Rev. C 90, 051301 (2014).

[50] J. Kotila and F. Iachello, "Phase space factors for double- $\beta$ decay," Phys. Rev. C 85, 034316 (2012).

[51] Sabin Stoica and Mihail Mirea, "New calculations for phase space factors involved in double- $\beta$ decay," Phys. Rev. C 88, 037303 (2013).

[52] F. Šimkovic, G. Pantis, J. D. Vergados, and A. Faessler, "Additional nucleon current contributions to neutrinoless double $\beta$ decay," Phys. Rev. C 60, 055502 (1999).

[53] V. A. Rodin, A. Faessler, F. Šimkovic, and P. Vogel, "Assessment of uncertainties in QRPA $0 \nu \beta \beta$-decay nuclear matrix elements," Nucl. Phys. A 766, 107 (2006), [Erratum: Nucl. Phys. A 793, 213 (2007)].

[54] Mihai Horoi and Sabin Stoica, "Shell model analysis of the neutrinoless double- $\beta$ decay of ${ }^{48} \mathrm{Ca}$," Phys. Rev. C 81, 024321 (2010).

[55] G.A. Miller and J.E. Spencer, "A survey of pion chargeexchange reactions with nuclei," Ann. Phys. 100, 562 (1976).

[56] R. Roth, H. Hergert, P. Papakonstantinou, T. Neff, and H. Feldmeier, "Matrix elements and few-body calculations within the unitary correlation operator method," Phys. Rev. C 72, 034002 (2005).

[57] K. A. Brueckner, "Many-body problem for strongly in- teracting particles. II. Linked cluster expansion," Phys. Rev. 100, 36-45 (1955).

[58] H. Müther and A. Polls, "Two-body correlations in nuclear systems," Prog. Part. Nucl. Phys. 45, 243-334 (2000).

[59] Omar Benhar, Riccardo Biondi, and Enrico Speranza, "Short-range correlation effects on the nuclear matrix element of neutrinoless double- $\beta$ decay," Phys. Rev. C 90, 065504 (2014).

[60] J. Engel, J. Carlson, and R.B. Wiringa, "Jastrow functions in double- $\beta$ decay," Phys. Rev. C 83, 034617 (2011).

[61] M. Kortelainen, O. Civitarese, J. Suhonen, and J. Toivanen, "Short-range correlations and neutrinoless double beta decay," Phys. Lett. B 647, 128 (2007).

[62] Fedor Šimkovic, Amand Faessler, Herbert Müther, Vadim Rodin, and Markus Stauf, "The $0 \nu \beta \beta$-decay nuclear matrix elements with self-consistent short-range correlations," Phys. Rev. C 79, 055501 (2009).

[63] J. Schechter and J. W. F. Valle, "Neutrino decay and spontaneous violation of lepton number," Phys. Rev. D 25, 25091 (1982).

[64] Werner Rodejohann, "Neutrino-less Double Beta Decay and Particle Physics," Int. J. Mod. Phys. E 20, 18331930 (2011).

[65] Frank F. Deppisch, Martin Hirsch, and Heinrich Pas, "Neutrinoless Double Beta Decay and Physics Beyond the Standard Model," J. Phys. G: Nucl. Part. Phys. 39, 124007 (2012).

[66] Andre de Gouvea and Petr Vogel, "Lepton Flavor and Number Conservation, and Physics Beyond the Standard Model," Prog. Part. Nucl. Phys. 71, 75-92 (2013).

[67] J. C. Helo, M. Hirsch, S. G. Kovalenko, and H. Pas, "Neutrinoless double beta decay and lepton number violation at the LHC," Phys. Rev. D 88, 011901 (2013).

[68] Heinrich Päs and Werner Rodejohann, "Neutrinoless Double Beta Decay," New J. Phys. 17, 115010 (2015).

[69] Tao Peng, Michael J. Ramsey-Musolf, and Peter Winslow, "TeV lepton number violation: From neutrinoless double- $\beta$ decay to the LHC," Phys. Rev. D 93, 093002 (2016).

[70] Mattias Blennow, Enrique Fernandez-Martinez, Jacobo Lopez-Pavon, and Javier Menéndez, "Neutrinoless double beta decay in seesaw models," JHEP 07, 096 (2010).

[71] R. Mohapatra and G. Senjanovic, "Neutrino mass and spontaneous parity nonconservation," Phys. Rev. Lett. 44, 912 (1980).

[72] R. Mohapatra and J. D. Vergados, "New contribution to neutrinoless double beta decay in gauge models," Phys. Rev. Lett. 47, 1713 (1981).

[73] R. Mohapatra, "New contributions to neutrinoless double-beta decay in supersymmetric theories," Phys. Rev. D 34, 3457 (1986).

[74] J. D. Vergados, "Neutrinoless Double Beta Decay Without Majorana Neutrinos in Supersymmetric Theories," Phys. Lett. B 184, 55-62 (1987).

[75] Y. Chikashigi, R. N. Mohapatra, and R. D. Peccei, "Are there real goldstone bosons associated with broken lepton number?" Phys. Lett. B 98, 265 (1981).

[76] G. B. Gelmini and M. Roncadelli, "Left-handed neutrino mass scale and spontaneously broken lepton number," Phys. Lett. B 99, 411 (1981).

[77] H. M. Georgi, S. L. Glashow, and S. Nussinov, "Unconventional model of neutrino masses," Nucl. Phys. B 
193, 297 (1981).

[78] Dusan Štefánik, Rastislav Dvornicky, Fedor Šimkovic, and Petr Vogel, "Reexamining the light neutrino exchange mechanism of the $0 \nu \beta \beta$ decay with left- and right-handed leptonic and hadronic currents," Phys. Rev. C 92, 055502 (2015).

[79] Amand Faessler, Marcela González, Sergey Kovalenko, and Fedor Šimkovic, "Arbitrary mass Majorana neutrinos in neutrinoless double beta decay," Phys. Rev. D 90, 096010 (2014).

[80] J. Barea, J. Kotila, and F. Iachello, "Limits on sterile neutrino contributions to neutrinoless double beta decay," Phys. Rev. D 92, 093001 (2015).

[81] Juhani Hyvärinen and Jouni Suhonen, "Nuclear matrix elements for $0 \nu \beta \beta$ decays with light or heavy Majorananeutrino exchange," Phys. Rev. C 91, 024613 (2015).

[82] Mihai Horoi and Andrei Neacsu, "Shell model predictions for ${ }^{124}$ Sn double- $\beta$ decay," Phys. Rev. C 93, 024308 (2016).

[83] J. Retamosa, E. Caurier, and F. Nowacki, "Neutrinoless double beta decay of ${ }^{48}$ Ca," Phys. Rev. C 51, 371-378 (1995).

[84] E. Caurier, F. Nowacki, A. Poves, and J. Retamosa, "Shell Model Studies of the Double Beta Decays of ${ }^{76} \mathrm{Ge},{ }^{82} \mathrm{Se}$, and ${ }^{136} \mathrm{Xe}$," Phys. Rev. Lett. 77, 1954-1957 (1996).

[85] Mihai Horoi and Andrei Neacsu, "Analysis of mechanisms that could contribute to neutrinoless double-beta decay," Phys. Rev. D 93, 113014 (2016).

[86] M. Hirsch, H. V. Klapdor-Kleingrothaus, and S. G. Kovalenko, "New constraints on $R$-parity broken supersymmetry from neutrinoless double beta decay," Phys. Rev. Lett. 75, 17-20 (1995).

[87] M. Hirsch, H.V. Klapdor-Kleingrothaus, and O. Panella, "Double beta decay in left-right symmetric models," Phys. Lett. B 374, 7 (1996).

[88] Mihai Horoi, "Shell model analysis of competing contributions to the double- $\beta$ decay of ${ }^{48} \mathrm{Ca}$," Phys. Rev. C 87, 014320 (2013).

[89] A. Meroni, S. T. Petcov, and F. Šimkovic, "Multiple CP non-conserving mechanisms of $(\beta \beta)_{0 \nu}$-decay and nuclei with largely different nuclear matrix elements," JHEP 02, 025 (2013).

[90] G. Prézeau, M. Ramsey-Musolf, and P. Vogel, "Neutrinoless double $\beta$ decay and effective field theory," Phys. Rev. D 68, 034016 (2003).

[91] A. Faessler, S. Kovalenko, F. Šimkovic, and J. Schwieger, "Dominance of pion exchange in $R$-parityviolating supersymmetric contributions to neutrinoless double beta decay," Phys. Rev. Lett. 78, 183 (1997).

[92] Amand Faessler, Sergey Kovalenko, and Fedor Šimkovic, "Pions in nuclei and manifestations of supersymmetry in neutrinoless double beta decay," Phys. Rev. D 58, 115004 (1998).

[93] Amand Faessler, Thomas Gutsche, Sergey Kovalenko, and Fedor Šimkovic, "Pion dominance in RPV SUSY induced neutrinoless double beta decay," Phys. Rev. D 77, 113012 (2008).

[94] Martin J. Savage, "Pionic matrix elements in neutrinoless double beta decay," Phys. Rev. C 59, 2293-2296 (1999).

[95] Amy Nicholson, Evan Berkowitz, Chia Cheng Chang, M. A. Clark, Balint Joo, Thorsten Kurth, Enrico Rinaldi, Brian Tiburzi, Pavlos Vranas, and Andre Walker-
Loud, "Neutrinoless double beta decay from lattice QCD," arXiv:1608.04793.

[96] S. K. Bogner, R. J. Furnstahl, and A. Schwenk, "From low-momentum interactions to nuclear structure," Prog. Part. Nucl. Phys. 65, 94-147 (2010).

[97] R. J. Furnstahl and K. Hebeler, "New applications of renormalization group methods in nuclear physics," Rept. Prog. Phys. 76, 126301 (2013).

[98] Gary Prézeau, "Light neutrino and heavy particle exchange in $0 \nu \beta \beta$-decay," Phys. Lett. B 633, 93 (2006).

[99] V. Cirigliano, A. Kurylov, M. J. Ramsey-Musolf, and P. Vogel, "Neutrinoless double beta decay and lepton flavor violation," Phys. Rev. Lett. 93, 231802 (2004).

[100] R. Arnold et al. (SuperNEMO Collaboration), "Probing New Physics Models of Neutrinoless Double Beta Decay with SuperNEMO," Eur. Phys. J. C 70, 927-943 (2010).

[101] A. Ali, A. V. Borisov, and D. V. Zhuridov, "Probing new physics in the neutrinoless double beta decay using electron angular correlation," Phys. Rev. D 76, 093009 (2007).

[102] Frank Deppisch and Heinrich Päs, "Pinning down the mechanism of neutrinoless double $\beta$ decay with measurements in different nuclei," Phys. Rev. Lett. 98, 232501 (2007).

[103] V. M. Gehman and S. R. Elliott, "Multiple-isotope comparison for determining $0 \nu \beta \beta$ mechanisms," J. Phys. G: Nucl. Part. Phys. 34, 667 (2007), [Erratum: J. Phys. G 35, 029701 (2008)].

[104] Fedor Šimkovic, John Vergados, and Amand Faessler, "Few active mechanisms of the neutrinoless double betadecay and effective mass of Majorana neutrinos," Phys. Rev. D 82, 113015 (2010).

[105] Amand Faessler, G. L. Fogli, E. Lisi, A.M. Rotunno, and F. Šimkovic, "Multi-isotope degeneracy of neutrinoless double beta decay mechanisms in the quasi-particle random phase approximation," Phys. Rev. D 83, 113015 (2011).

[106] E. Lisi, A. Rotunno, and F. Šimkovic, "Degeneracies of particle and nuclear physics uncertainties in neutrinoless $\beta \beta$ decay," Phys. Rev. D 92, 093004 (2015).

[107] F. Šimkovic, M. Nowak, W. A. Kaminski, A. A. Raduta, and Amand Faessler, "Neutrinoless double beta decay of ${ }^{76} \mathrm{Ge},{ }^{82} \mathrm{Se},{ }^{100} \mathrm{Mo}$ and ${ }^{136} \mathrm{Xe}$ to excited $0^{+}$states," Phys. Rev. C 64, 035501 (2001).

[108] J. Menéndez, A. Poves, E. Caurier, and F. Nowacki, "Occupancies of individual orbits, and the nuclear matrix element of the ${ }^{76}$ Ge neutrinoless $\beta \beta$ decay," Phys. Rev. C 80, 048501 (2009).

[109] J. Barea, J. Kotila, and F. Iachello, " $0 \nu \beta \beta$ and $2 \nu \beta \beta$ nuclear matrix elements in the interacting boson model with isospin restoration," Phys. Rev. C 91, 034304 (2015).

[110] Juhani Hyvärinen and Jouni Suhonen, "Neutrinoless $\beta \beta$ decays to excited $0^{+}$states and the Majorana-neutrino mass," Phys. Rev. C 93, 064306 (2016).

[111] J. J. Gomez-Cadenas, J. Martin-Albo, M. Sorel, P. Ferrario, F. Monrabal, J. Munoz-Vidal, P. Novella, and A. Poves, "Sense and sensitivity of double beta decay experiments," JCAP 1106, 007 (2011).

[112] R. G. H. Robertson, "Empirical survey of neutrinoless double beta decay matrix elements," Mod. Phys. Lett. A 28, 1350021 (2013).

[113] J. Menéndez, A. Poves, E. Caurier, and F. Nowacki, "Disassembling the nuclear matrix elements of the neu- 
trinoless double beta decay," Nucl. Phys. A 818, 139151 (2009).

[114] Y. Iwata, N. Shimizu, T. Otsuka, Y. Utsuno, J. Menéndez, M. Honma, and T. Abe, "Large-scale shell-model analysis of the neutrinoless $\beta \beta$ decay of ${ }^{48}$ Ca," Phys. Rev. Lett. 116, 112502 (2016).

[115] Fedor Šimkovic, Vadim Rodin, Amand Faessler, and Petr Vogel, " $0 \nu \beta \beta$ and $2 \nu \beta \beta$ nuclear matrix elements, quasiparticle ramdom-phase approximation, and isospin symmetry restoration," Phys. Rev. C 87, 045501 (2013).

[116] Dong-Liang Fang, Amand Faessler, and Fedor Šmkovic, "Partial restoration of isospin symmetry for neutrinoless double $\beta$ decay in the deformed nuclear system of ${ }^{150} \mathrm{Nd}$," Phys. Rev. C 92, 044301 (2015).

[117] M. T. Mustonen and J. Engel, "Large-scale calculations of the double- $\beta$ decay of ${ }^{76} \mathrm{Ge},{ }^{128} \mathrm{Te},{ }^{136} \mathrm{Xe}$, and ${ }^{150} \mathrm{Nd}$ in the deformed self-consistent Skyrme quasiparticle random-phase approximation," Phys. Rev. C 87, 064302 (2013).

[118] J. M. Yao, L. S. Song, K. Hagino, P. Ring, and J. Meng, "Systematic study of nuclear matrix elements in neutrinoless double- $\beta$ decay with a beyond-mean-field covariant density functional theory," Phys. Rev. C 91, 024316 (2015).

[119] J. M. Yao and J. Engel, "Octupole correlations in lowlying states of ${ }^{150} \mathrm{Nd}$ and ${ }^{150} \mathrm{Sm}$ and their impact on neutrinoless double- $\beta$ decay," Phys. Rev. C 94, 014306 (2016).

[120] Nuria López Vaquero, Tomás R. Rodríguez, and J. Luis Egido, "Shape and pairing fluctuations effects on neutrinoless double beta decay," Phys. Rev. Lett. 111, 142501 (2013).

[121] E. Caurier, G. Martínez-Pinedo, F. Nowacki, A. Poves, and A. P. Zuker, "The shell model as a unified view of nuclear structure," Rev. Mod. Phys. 77, 427 (2005).

[122] J. Barea, J. Kotila, and F. Iachello, "Nuclear matrix elements for double- $\beta$ decay," Phys. Rev. C 87, 014315 (2013).

[123] Pekka Pirinen and Jouni Suhonen, "Systematic approach to $\beta$ and $2 \nu \beta \beta$ decays of mass $A=100-136$ nuclei," Phys. Rev. C 91, 054309 (2015).

[124] B. A. Brown, "The nuclear shell model towards the drip lines," Prog. Part. Nucl. Phys. 47, 517 - 599 (2001).

[125] T. Otsuka, M. Honma, T. Mizusaki, N. Shimizu, and Y. Utsuno, "Monte carlo shell model for atomic nuclei," Prog. Part. Nucl. Phys. 47, 319 - 400 (2001).

[126] P. Ćársky, "Configuration interaction," in Encyclopedia of Computation Chemistry, edited by P. Schleyer, N. L. Allinger, T. Clark, J. Gasteiger, P. A. Kollman, H. F. Schaefer, and P. R. Schreiner (John Wiley and Sons, Chichester, 1998) pp. 485-497.

[127] C. David Sherrill and Henry F. Schaefer, "The configuration interaction method: Advances in highly correlated approaches," Adv. Quantum Chem. 34, 143 - 269 (1999).

[128] Marianne Dufour and Andrés P. Zuker, "The realistic collective nuclear hamiltonian," Phys. Rev. C 54, 16411660 (1996).

[129] M. G. Mayer, "On closed shells in nuclei. II," Phys. Rev. 75, 1969 (1949).

[130] O. Haxel, J. H. D. Jensen, and H. E. Suess, "On the "magic numbers" in nuclear structure," Phys. Rev. 75, 1766 (1949).

[131] Noritaka Shimizu, Yutaka Utsuno, Yasunori Futamura,
Tetsuya Sakurai, Takahiro Mizusaki, and Takaharu Otsuka, "Stochastic estimation of nuclear level density in the nuclear shell model: An application to paritydependent level density in ${ }^{58} \mathrm{Ni}$," Phys. Lett. B 753, 13 (2016).

[132] F. Nowacki, A. Poves, E. Caurier, and B. Bounthong, "Shape Coexistence in ${ }^{78} \mathrm{Ni}$ as the Portal to the Fifth Island of Inversion," Phys. Rev. Lett. 117, 272501 (2016).

[133] E. Caurier, J. Menéndez, F. Nowacki, and A. Poves, "The influence of pairing on the nuclear matrix elements of the neutrinoless double beta decays," Phys. Rev. Lett. 100, 052503 (2008).

[134] Andrei Neacsu and Mihai Horoi, "Shell model studies of the ${ }^{130}$ Te neutrinoless double-beta decay," Phys. Rev. C 91, 024309 (2015).

[135] R. A. Sen'kov and M. Horoi, "Shell-model calculation of neutrinoless double- $\beta$ decay of ${ }^{76} \mathrm{Ge}, "$ Phys. Rev. C 93, 044334 (2016).

[136] Petr Vogel, "Nuclear structure and double beta decay," J. Phys. G: Nucl. Part. Phys. 39, 124002 (2012).

[137] W. T. Chou, E. K. Warburton, and B. Alex Brown, "Gamow-Teller beta-decay rates for A $\leq 18$ nuclei," Phys. Rev. C 47, 163-177 (1993).

[138] B. H. Wildenthal, M. S. Curtin, and B. Alex Brown, "Predicted features of the beta decay of neutron-rich sd-shell nuclei," Phys. Rev. C 28, 1343-1366 (1983).

[139] G. Martínez-Pinedo, A. Poves, E. Caurier, and A. P. Zuker, "Effective $g_{A}$ in the pf shell," Phys. Rev. C 53, R2602 (1996).

[140] E. Caurier, A. P. Zuker, and A. Poves, "A Full $0 \hbar \omega$ description of the $2 \nu \beta \beta$ decay of ${ }^{48} \mathrm{Ca}$," Phys. Lett. B 252, 13-17 (1990).

[141] A. Poves, R. P. Bahukutumbi, K. Langanke, and P. Vogel, "Double beta decay of ${ }^{48}$ Ca revisited," Phys. Lett. B 361, 1-4 (1995).

[142] R. Arnold et al. (NEMO-3 Collaboration), "Measurement of the double-beta decay half-life and search for the neutrinoless double-beta decay of ${ }^{48} \mathrm{Ca}$ with the NEMO-3 detector," Phys. Rev. D 93, 112008 (2016).

[143] A. Balysh, Asoka S. De Silva, V. I. Lebedev, K. Lou, M. K. Moe, M. A. Nelson, A. Piepke, A. Pronsky, M. A. Vient, and P. Vogel, "Double beta decay of ${ }^{48} \mathrm{Ca}$," Phys. Rev. Lett. 77, 5186-5189 (1996).

[144] Etienne Caurier, Frederic Nowacki, and Alfredo Poves, "Shell Model description of the $\beta \beta$ decay of ${ }^{136} \mathrm{Xe}$," Phys. Lett. B 711, 62-64 (2012).

[145] M. Horoi and B.A. Brown, "Shell-model analysis of the ${ }^{136}$ Xe double beta decay nuclear matrix elements," Phys. Rev. Lett. 110, 222502 (2013).

[146] I.S. Towner, "Quenching of spin matrix elements in nuclei," Phys. Rep. 155, 263 (1997).

[147] B. A. Brown and B. H. Wildenthal, "Empirically optimum M1 operator for sd-shell nuclei," Nucl. Phys. A 474, 290-306 (1987).

[148] P. von Neumann-Cosel, A. Poves, J. Retamosa, and A. Richter, "Magnetic dipole response in nuclei at the $N=28$ shell," Phys. Lett. B 443, 1-6 (1998).

[149] M. Hjorth-Jensen, T.T.S. Kuo, and E. Osnes, "Realistic effective interactions for nuclear systems," Phys. Rep. 261, 125 (1995).

[150] David Bohm and David Pines, "A Collective Description of Electron Interactions. I. Magnetic Interactions," Phys. Rev. 82, 625-634 (1951).

[151] D. J. Rowe, "Equations-of-motion method and the ex- 
tended shell model," Rev. Mod. Phys. 40, 153 (1968).

[152] B. Jancovici and D. H. Schiff, "The collective vibrations of a many-fermion system," Nucl. Phys. 58, 678-686 (1964).

[153] Peter Ring and Peter Schuck, The Nuclear ManyBody Problem (Springer-Verlag, New York, Heidelberg, Berlin, 1980).

[154] V.A. Rodin, Amand Faessler, F. Šimkovic, and Petr Vogel, "Uncertainty in the $0 \nu \beta \beta$ decay nuclear matrix elements," Phys. Rev. C 68, 044302 (2003).

[155] P. Vogel and M. R. Zirnbauer, "Suppression of the two-neutrino double-beta decay by nuclear-structure effects," Phys. Rev. Lett. 57, 3148-3151 (1986).

[156] J. Engel, P. Vogel, and M. R. Zirnbauer, "Nuclear structure effects in double-beta decay," Phys. Rev. C 37, 731 (1988).

[157] J. Toivanen and J. Suhonen, "Renormalized protonneutron quasiparticle random-phase approximation and its application to double beta decay," Phys. Rev. Lett. 75, 410 (1995).

[158] Vadim Rodin and Amand Faessler, "Fully renormalized quasiparticle random-phase approximation fulfills Ikeda sum rule exactly," Phys. Rev. C 66, 051303 (2002).

[159] J. Engel, S. Pittel, M. Stoitsov, P. Vogel, and J. Dukelsky, "Neutron-proton correlations in an exactly solvable model," Phys. Rev. C 55, 1781 (1997).

[160] Jorge G. Hirsch, Peter O. Hess, and Osvaldo Civitarese, "Single- and Double-Beta Decay Fermi Transitions in an Exactly Solvable Model," Phys. Rev. C 56, 199 (1997).

[161] J. Terasaki, "Many-body correlations of quasiparticle random-phase approximation in nuclear matrix elements of neutrinoless double- $\beta$ decay," Phys. Rev. C 91, 034318 (2015).

[162] Jun Terasaki, "Two decay paths for calculating the nuclear matrix element of neutrinoless double- $\beta$ decay using quasiparticle random-phase approximation," Phys. Rev. C 93, 024317 (2016).

[163] Fernando Nogueira, Miguel A L Marques, and Carlos Fiolhais, A primer in density functional theory, Lecture Notes in Physics (Springer, Berlin, 2003).

[164] P. Hohenberg and W. Kohn, "Inhomogeneous Electron Gas," Phys. Rev. 136, B864-B871 (1964).

[165] W. Kohn and L. J. Sham, "Self-Consistent Equations Including Exchange and Correlation Effects," Phys. Rev. 140, A1133-A1138 (1965).

[166] D. Vautherin and D. M. Brink, "Hartree-Fock calculations with Skyrme's interaction. 1. Spherical nuclei," Phys. Rev. C 5, 626-647 (1972).

[167] J. Dechargé and D. Gogny, "Hartre-fock-bogolyubov calculations with the d1 effective interaction on spherical nuclei," Phys. Rev. C 21, 1568-1593 (1980).

[168] Brian D. Serot and John Dirk Walecka, "The Relativistic Nuclear Many Body Problem," Adv. Nucl. Phys. 16, 1-327 (1986).

[169] Michael Bender, Paul-Henri Heenen, and Paul-Gerhard Reinhard, "Self-consistent mean-field models for nuclear structure," Rev. Mod. Phys. 75, 121-180 (2003).

[170] M. Bender, T. Duguet, and D. Lacroix, "ParticleNumber Restoration within the Energy Density Functional Formalism," Phys. Rev. C 79, 044319 (2009).

[171] M. Anguiano, J. L. Egido, and L. M. Robledo, "Particle number projection with effective forces," Nucl. Phys. A 696, 467-493 (2001).

[172] J. Dobaczewski, M. V. Stoitsov, W. Nazarewicz, and
P. G. Reinhard, "Particle-Number Projection and the Density Functional Theory," Phys. Rev. C 76, 054315 (2007).

[173] Tomás R. Rodríguez and G. Martínez-Pinedo, "Energy density functional study of nuclear matrix elements for neutrinoless $\beta \beta$ decay," Phys. Rev. Lett. 105, 252503 (2010).

[174] L. S. Song, J. M. Yao, P. Ring, and J. Meng, "Relativistic description of nuclear matrix elements in neutrinoless double- $\beta$ decay," Phys. Rev. C 90, 054309 (2014).

[175] Javier Menéndez, Tomás R. Rodríguez, Gabriel Martínez-Pinedo, and Alfredo Poves, "Correlations and neutrinoless $\beta \beta$ decay nuclear matrix elements of $p f$ shell nuclei," Phys. Rev. C 90, 024311 (2014).

[176] Javier Menéndez, Nobuo Hinohara, Jonathan Engel, Gabriel Martínez-Pinedo, and Tomás R. Rodríguez, "Testing the importance of collective correlations in neutrinoless decay," Phys. Rev. C 93, 014305 (2016).

[177] Nobuo Hinohara and Jonathan Engel, "Proton-Neutron Pairing Amplitude as a Generator Coordinate for Double-Beta Decay," Phys. Rev. C 90, 031301 (2014).

[178] Franceso Iachello and Akito Arima, The interacting boson model (Cambridge University Press, Cambridge, 1987).

[179] A. Arima and F. Iachello, "The interacting boson model," Ann. Rev. Nucl. Part. Sci. 31, 75-105 (1981).

[180] Aage Bohr and Ben R. Mottelson, Nuclear Structure, Vol. 2 (World Scientific Pub Co Inc, 1998).

[181] T. Otsuka, A. Arima, and F. Iachello, "Nuclear shell model and interacting bosons," Nucl. Phys. A 309, 1-33 (1978).

[182] J. Barea and F. Iachello, "Neutrinoless double- $\beta$ decay in the microscopic interacting boson model," Phys. Rev. C 79, 044301 (2009).

[183] K. Chaturvedi, R. Chandra, P. K. Rath, P. K. Raina, and J. G. Hirsch, "Nuclear deformation and neutrinoless double- $\beta$ decay of ${ }^{94} \mathrm{Zr},{ }^{96} \mathrm{Zr},{ }^{98} \mathrm{Mo},{ }^{100} \mathrm{Mo},{ }^{104} \mathrm{Ru}$, ${ }^{110} \mathrm{Pd},{ }^{128} \mathrm{Te},{ }^{130} \mathrm{Te}$, and ${ }^{150} \mathrm{Nd}$ nuclei within a mechanism involving neutrino mass," Phys. Rev. C 78, 054302 (2008).

[184] R. Chandra, K. Chaturvedi, P. K. Rath, P. K. Raina, and J. G. Hirsch, "Multipolar correlations and deformation effect on nuclear transition matrix elements of double-beta decay," Europhys. Lett. 86, 32001 (2009).

[185] P. K. Rath, R. Chandra, K. Chaturvedi, P. K. Raina, and J. G. Hirsch, "Uncertainties in nuclear transition matrix elements for neutrinoless $\beta \beta$ decay within the PHFB model," Phys. Rev. C 82, 064310 (2010).

[186] P. K. Rath, R. Chandra, K. Chaturvedi, P. Lohani, P. K. Raina, and J. G. Hirsch, "Neutrinoless decay transition matrix elements within mechanisms involving light Majorana neutrinos, classical Majorons, and sterile neutrinos," Phys. Rev. C 88, 064322 (2013).

[187] J. G. Hirsch, O. Castaños, P. O. Hess, and O. Civitarese, "Pseudo SU(3) approach to the $\beta \beta$ decay," Prog. Part. Nucl. Phys. 32, 333-334 (1994).

[188] J. G. Hirsch, O. Castaños, and P. O. Hess, "Neutrinoless double beta decay in heavy deformed nuclei," Nucl. Phys. A 582, 124 (1995).

[189] E. Caurier, F. Nowacki, and A. Poves, "Nuclear Structure Aspects of the Neutrinoless Double Beta Decay," Eur. Phys. J. A 36, 195-200 (2008).

[190] A. A. Kwiatkowski et al., "New Determination of Double-Beta-Decay Properties in ${ }^{48} \mathrm{Ca}$ : High-Precision 
Q-Value Measurement and Improved Nuclear Matrix Element Calculations," Phys. Rev. C 89, 045502 (2014).

[191] Jason D. Holt and Jonathan Engel, "Effective double$\beta$-decay operator for ${ }^{76} \mathrm{Ge}$ and ${ }^{82} \mathrm{Se}, "$ Phys. Rev. C 87 , 064315 (2013).

[192] Alberto Escuderos, Amand Faessler, Vadim Rodin, and Fedor Šimkovic, "Contributions of different neutron pairs in different approaches for neutrinoless double beta decay," J. Phys. G: Nucl. Part. Phys. 37, 125108 (2010).

[193] J. Menéndez, A. Poves, E. Caurier, and F. Nowacki, "Neutrinoless double beta decay: The nuclear matrix elements revisited," J. Phys. Conf. Ser. 312, 072005 (2011).

[194] Fedor Šimkovic, Amand Faessler, Vadim Rodin, Petr Vogel, and Jonathan Engel, "Anatomy of the $0 \nu \beta \beta \mathrm{nu}-$ clear matrix elements," Phys. Rev. C 77, 045503 (2008).

[195] Tomás R. Rodríguez and J. Luis Egido, "New BeyondMean-Field Theories: Examination of the Potential Shell Closures at $\mathrm{N}=32$ or 34," Phys. Rev. Lett. 99, 062501 (2007).

[196] A. Poves, J. Sánchez-Solano, E. Caurier, and F. Nowacki, "Shell model study of the isobaric chains $\mathrm{A}=50, \mathrm{~A}=51$ and $\mathrm{A}=52$," Nucl. Phys. A 694, 157198 (2001).

[197] J. Menéndez, A. Poves, E. Caurier, and F. Nowacki, "Novel nuclear structure aspects of the $0 \nu \beta \beta$-decay," J. Phys. Conf. Ser. 267, 012058 (2011).

[198] Dong-Liang Fang, Amand Faessler, Vadim Rodin, and Fedor Šimkovic, "Neutrinoless Double Beta Decay of Deformed Nuclei within QRPA with Realistic Interaction," Phys. Rev. C 83, 034329 (2011).

[199] B. Alex Brown and B. H. Wildenthal, "Status of the nuclear shell model," Ann. Rev. Nucl. Part. Sci. 38, 29-66 (1988).

[200] Vikas Kumar, P. C. Srivastava, and Hantao Li, "Nuclear $\beta^{-}$-decay half-lives for $f p$ and $f p g$ shell nuclei," J. Phys. G: Nucl. Part. Phys. 43, 105104 (2016).

[201] B. Alex Brown, W. Chung, and B. H. Wildenthal, "Empirical Renormalization of the One-Body Gamow-Teller beta-Decay Matrix Elements in the 1s-0d Shell," Phys. Rev. Lett. 40, 1631-1635 (1978).

[202] G. F. Bertsch and I. Hamamoto, "Gamow-Teller strength at high excitations," Phys. Rev. C 26, 13231326 (1982).

[203] A. Arima, K. Shimizu, W. Bentz, and H. Hyuga, "Nuclear Magnetic Properties and Gamow-teller Transitions," Adv. Nucl. Phys. 18, 1-106 (1987).

[204] E. Caurier, A. Poves, and A. P. Zuker, "Missing and quenched Gamow-Teller strength," Phys. Rev. Lett. 74, 1517-1520 (1995).

[205] T.-S. Park, H. Jung, and D.-P. Min, "In-medium effective axial-vector coupling constant," Phys. Lett. B 409, 26-32 (1997).

[206] K. Ikeda, S. Fujii, and J. I. Fujita, "The (p,n) reactions and beta decays," Phys. Lett. 3, 271 - 272 (1963).

[207] M. Ichimura, H. Sakai, and T. Wakasa, "Spin-isospin responses via (p,n) and (n,p) reactions," Prog. Part. Nucl. Phys. 56, 446-531 (2006).

[208] Y. Fujita, B. Rubio, and W. Gelletly, "Spin-isospin excitations probed by strong, weak and electro-magnetic interactions," Prog. Part. Nucl. Phys. 66, 549-606 (2011).

[209] D. Frekers, P. Puppe, J. H. Thies, and H. Ejiri,
"Gamow-Teller strength extraction from $\left({ }^{3} \mathrm{He}, t\right)$ reactions," Nucl. Phys. A 916, 219-240 (2013).

[210] K. Yako et al., "Gamow-Teller Strength Distributions in ${ }^{48} \mathrm{Sc}$ by the ${ }^{48} \mathrm{Ca}(\mathrm{p}, \mathrm{n})$ and ${ }^{48} \mathrm{Ti}(\mathrm{n}, \mathrm{p})$ Reactions and TwoNeutrino Double- $\beta$ Decay Nuclear Matrix Elements," Phys. Rev. Lett. 103, 012503 (2009).

[211] Y. Fujita et al., "High-resolution study of $T_{z}=+2 \rightarrow$ +1 Gamow-Teller transitions in the ${ }^{44} \mathrm{Ca}\left({ }^{3} \mathrm{He}, \mathrm{t}\right){ }^{44} \mathrm{Sc}$ reaction," Phys. Rev. C 88, 014308 (2013).

[212] S. Noji et al., " $\beta^{+}$Gamow-Teller Transition Strengths from ${ }^{46} \mathrm{Ti}$ and Stellar Electron-Capture Rates," Phys. Rev. Lett. 112, 252501 (2014).

[213] Y. Iwata, N. Shimizu, Y. Utsuno, M. Honma, T. Abe, and T. Otsuka, "Ingredients of nuclear matrix element for two-neutrino double-beta decay of ${ }^{48} \mathrm{Ca}$," JPS Conf. Proc. 6, 030057 (2015).

[214] K. Yako et al., "Determination of the Gamow-Teller quenching factor from charge exchange reactions on ${ }^{90}$ Zr," Phys. Lett. B 615, 193-199 (2005).

[215] M. Sasano et al., "Gamow-Teller unit cross sections of the $(\mathrm{p}, \mathrm{n})$ reaction at 198 and $297 \mathrm{MeV}$ on mediumheavy nuclei," Phys. Rev. C 79, 024602 (2009).

[216] H. Ejiri and D. Frekers, "Spin dipole nuclear matrix elements for double beta decay nuclei by charge-exchange reactions," J. Phys. G: Nucl. Part. Phys. 43, 11LT01 (2016).

[217] J. Menéndez, D. Gazit, and A. Schwenk, "Chiral twobody currents in nuclei: Gamow-teller transitions and neutrinoless double-beta decay," Phys. Rev. Lett. 107, 062501 (2011).

[218] J. Engel, F. Šimkovic, and P. Vogel, "Chiral Two-Body Currents and Neutrinoless Double-Beta Decay in the QRPA," Phys. Rev. C 89, 064308 (2014).

[219] Vijay R. Pandharipande, Ingo Sick, and Peter K. A. deWitt Huberts, "Independent particle motion and correlations in fermion systems," Rev. Mod. Phys. 69, 981991 (1997).

[220] T. Siiskonen, M. Hjorth-Jensen, and J. Suhonen, "Renormalization of the weak hadronic current in the nuclear medium," Phys. Rev. C 63, 055501 (2001).

[221] T. S. Park, L. E. Marcucci, R. Schiavilla, M. Viviani, A. Kievsky, S. Rosati, K. Kubodera, D. P. Min, and M. Rho, "Parameter free effective field theory calculation for the solar proton fusion and hep processes," Phys. Rev. C 67, 055206 (2003).

[222] Martin Hoferichter, Philipp Klos, and Achim Schwenk, "Chiral power counting of one- and two-body currents in direct detection of dark matter," Phys. Lett. B 746, 410-416 (2015).

[223] H. Krebs, E. Epelbaum, and U. G. Meiner, "Nuclear axial current operators to fourth order in chiral effective field theory," Annals Phys. 378, 317-395 (2017).

[224] Malcolm Butler, Jiunn-Wei Chen, and Xinwei Kong, "Neutrino deuteron scattering in effective field theory at next-to-next-to-leading order," Phys. Rev. C 63, 035501 (2001).

[225] S. Nakamura, T. Sato, Vladimir P. Gudkov, and K. Kubodera, "Neutrino reactions on the deuteron," Phys. Rev. C 63, 034617 (2001).

[226] Doron Gazit, Sofia Quaglioni, and Petr Navrátil, "Three-Nucleon Low-Energy Constants from the Consistency of Interactions and Currents in Chiral Effective Field Theory," Phys. Rev. Lett. 103, 102502 (2009).

[227] A. Baroni, L. Girlanda, A. Kievsky, L. E. Marcucci, 
R. Schiavilla, and M. Viviani, "Tritium $\beta$-decay in chiral effective field theory," Phys. Rev. C 94, 024003 (2016).

[228] Sonia Bacca and Saori Pastore, "Electromagnetic reactions on light nuclei," J. Phys. G: Nucl. Part. Phys. 41, 123002 (2014).

[229] L. E. Marcucci, F. Gross, M. T. Pena, M. Piarulli, R. Schiavilla, I. Sick, A. Stadler, J. W. Van Orden, and M. Viviani, "Electromagnetic Structure of FewNucleon Ground States," J. Phys. G: Nucl. Part. Phys. 43, 023002 (2016).

[230] P. Klos, J. Menéndez, D. Gazit, and A. Schwenk, "Large-scale nuclear structure calculations for spindependent WIMP scattering with chiral effective field theory currents," Phys. Rev. D 88, 083516 (2013), [Erratum: Phys. Rev. D 89, 029901 (2014)].

[231] J. Menéndez, "What do we know about neutrinoless double-beta decay nuclear matrix elements?" arXiv:1605.05059.

[232] Nikolaj Thomas Zinner, Karlheinz Langanke, and Petr Vogel, "Muon capture on nuclei: Random phase approximation evaluation versus data for $6 \leq Z \leq 94$ nuclei," Phys. Rev. C 74, 024326 (2006).

[233] A. C. Hayes and I. S. Towner, "Shell model calculations of neutrino scattering from C-12," Phys. Rev. C 61, 044603 (2000).

[234] C. Volpe, N. Auerbach, G. Colo, T. Suzuki, and N. Van Giai, "Microscopic theories of neutrino C-12 reactions," Phys. Rev. C 62, 015501 (2000).

[235] Toshio Suzuki, Satoshi Chiba, Takashi Yoshida, Toshitaka Kajino, and Takaharu Otsuka, "Neutrino nucleus reactions based on new shell model Hamiltonians," Phys. Rev. C 74, 034307 (2006).

[236] A. C. Hayes, P. Navrátil, and J. P. Vary, "Neutrino C12 scattering in the ab initio shell model with a realistic three body interaction," Phys. Rev. Lett. 91, 012502 (2003).

[237] A. Lovato, S. Gandolfi, J. Carlson, Steven C. Pieper, and R. Schiavilla, "Electromagnetic response of ${ }^{12} \mathrm{C}$ : A first-principles calculation," Phys. Rev. Lett. 117, 082501 (2016).

[238] A. Ekström, G. R. Jansen, K. A. Wendt, G. Hagen, T. Papenbrock, S. Bacca, B. Carlsson, and D. Gazit, "Effects of three-nucleon forces and two-body currents on Gamow-Teller strengths," Phys. Rev. Lett. 113, 262504 (2014).

[239] Noritaka Shimizu, Takashi Abe, Yusuke Tsunoda, Yutaka Utsuno, Tooru Yoshida, Takahiro Mizusaki, Michio Honma, and Takaharu Otsuka, "New-generation Monte Carlo shell model for the K computer era," Prog. Theor. Exp. Phys. 2012, 01A205 (2012).

[240] Christina Stumpf, Jonas Braun, and Robert Roth, "Importance-Truncated Large-Scale Shell Model," Phys. Rev. C 93, 021301 (2016).

[241] Ö. Legeza, L. Veis, A. Poves, and J. Dukelsky, "Advanced density matrix renormalization group method for nuclear structure calculations," Phys. Rev. C 92, 051303 (2015).

[242] Tomoaki Togashi, Yusuke Tsunoda, Takaharu Otsuka, and Noritaka Shimizu, "Quantum Phase Transition in the Shape of Zr isotopes," Phys. Rev. Lett. 117, 172502 (2016).

[243] Fedor Šimkovic, Amand Faessler, and Petr Vogel,
" $0 \nu \beta \beta$ nuclear matrix elements and the occupancy of individual orbits," Phys. Rev. C 79, 015502 (2009).

[244] Tomás R. Rodríguez, Alfredo Poves, and Frdric Nowacki, "Occupation numbers of spherical orbits in self-consistent beyond-mean-field methods," Phys. Rev. C 93, 054316 (2016).

[245] T. Abe, P. Maris, T. Otsuka, N. Shimizu, Y. Utsuno, and J. P. Vary, "Benchmarks of the full configuration interaction, Monte Carlo shell model, and no-core full configuration methods," Phys. Rev. C 86, 054301 (2012).

[246] Petr Navrátil, Sofia Quaglioni, Guillaume Hupin, Carolina Romero-Redondo, and Angelo Calci, "Unified ab initio approaches to nuclear structure and reactions," Phys. Scr. 91, 053002 (2016).

[247] Bruce R. Barrett, Petr Navrátil, and James P. Vary, "Ab initio no core shell model," Prog. Part. Nucl. Phys. 69, 131-181 (2013).

[248] Andrés P. Zuker, "Three body monopole corrections to the realistic interactions," Phys. Rev. Lett. 90, 042502 (2003).

[249] T. Otsuka, T. Suzuki, J.D. Holt, A. Schwenk, and Y. Akaishi, "Three-body forces and the limit of oxygen isotopes," Phys. Rev. Lett 105, 032501 (2010).

[250] G. R. Jansen, J. Engel, G. Hagen, P. Navrátil, and A. Signoracci, "Ab-initio coupled-cluster effective interactions for the shell model: Application to neutron-rich oxygen and carbon isotopes," Phys. Rev. Lett. 113, 142502 (2014).

[251] S. K. Bogner, H. Hergert, J. D. Holt, A. Schwenk, S. Binder, A. Calci, J. Langhammer, and R. Roth, "Nonperturbative shell-model interactions from the inmedium similarity renormalization group," Phys. Rev. Lett. 113, 142501 (2014).

[252] E. Dikmen, A. F. Lisetski, B. R. Barrett, P. Maris, A. M. Shirokov, and J. P. Vary, "Ab initio effective interactions for sd-shell valence nucleons," Phys. Rev. C 91, 064301 (2015).

[253] Naofumi Tsunoda, Takaharu Otsuka, Noritaka Shimizu, Morten Hjorth-Jensen, Kazuo Takayanagi, and Toshio Suzuki, "Exotic neutron-rich medium-mass nuclei with realistic nuclear forces," Phys. Rev. C 95, 021304 (2017).

[254] J. A. Sheikh, N. Hinohara, J. Dobaczewski, T. Nakatsukasa, W. Nazarewicz, and K. Sato, "Isospin-invariant Skyrme energy-density-functional approach with axial symmetry," Phys. Rev. C 89, 054317 (2014).

[255] P. van Isacker, J. Engel, and K. Nomura, In preparation.

[256] A. A. Raduta, A. Faessler, and S. Stoica, "The $2 \nu \beta \beta$ decay rate within a boson expansion formalism," Nucl. Phys. A 534, 149 (1991).

[257] S. Stoica and H. V. Klapdor-Kleingrothaus, "Critical view on double-beta decay matrix elements within quasi random phase approximation-based methods," Nucl. Phys. A 694, 269 (2001).

[258] P. Papakonstantinou and R. Roth, "Large-scale second RPA calculations with finite-range interactions," Phys. Rev. C 81, 024317 (2010).

[259] D. Gambacurta, M. Grasso, and F. Catara, "Collective nuclear excitations with Skyrme-second RPA," Phys. Rev. C 81, 054312 (2010).

[260] D. Gambacurta, M. Grasso, and J. Engel, "Subtraction method in the second random-phase approximation: first applications with a Skyrme energy func- 
tional," Phys. Rev. C 92, 034303 (2015).

[261] E. Litvinova, P. Ring, and V. Tselyaev, "Relativistic quasiparticle time blocking approximation. Dipole response of open-shell nuclei," Phys. Rev. C 78, 014312 (2008).

[262] Caroline Robin and Elena Litvinova, "Nuclear response theory for spin-isospin excitations in a relativistic quasiparticle-phonon coupling framework," Eur. Phys. J. A 52, 205 (2016).

[263] J. Terasaki, "Overlap of quasiparticle random-phase approximation states for nuclear matrix elements of the neutrino-less double-beta decay," Phys. Rev. C 86, 021301 (2012).

[264] J. Terasaki, "Overlap of quasiparticle random-phase approximation states based on ground states of different nuclei: Mathematical properties and test calculations," Phys. Rev. C 87, 024316 (2013).

[265] S. R. Beane, W. Detmold, K. Orginos, and M. J. Savage, "Nuclear Physics from Lattice QCD," Prog. Part. Nucl. Phys. 66, 1-40 (2011).

[266] Sinya Aoki, Takumi Doi, Tetsuo Hatsuda, Yoichi Ikeda, Takashi Inoue, Noriyoshi Ishii, Keiko Murano, Hidekatsu Nemura, and Kenji Sasaki (HAL QCD Collaboration), "Lattice QCD approach to Nuclear Physics," PTEP 2012, 01A105 (2012).

[267] Raúl A. Briceño, Zohreh Davoudi, and Thomas C. Luu, "Nuclear Reactions from Lattice QCD," J. Phys. G: Nucl. Part. Phys. 42, 023101 (2015).

[268] A. Abdel-Rehim et al., "Nucleon and pion structure with lattice QCD simulations at physical value of the pion mass," Phys. Rev. D 92, 114513 (2015), [Erratum: Phys. Rev.D93,no.3,039904(2016)].

[269] R. B. Wiringa, V. G. J. Stoks, and R. Schiavilla, "Accurate nucleon-nucleon potential with chargeindependence breaking," Phys. Rev. C 51, 38 (1995).

[270] R. Machleidt, K. Holinde, and C. Elster, "The Bonn meson exchange model for the nucleon nucleon interaction," Phys. Rep. 149, 1-89 (1987).

[271] V. G. J. Stoks, R. A. M. Klomp, C. P. F. Terheggen, and J. J. de Swart, "Construction of High Quality NN Potential Models," Phys. Rev. C 49, 2950-2962 (1994).

[272] S. Gandolfi, A. Lovato, J. Carlson, and Kevin E. Schmidt, "From the lightest nuclei to the equation of state of asymmetric nuclear matter with realistic nuclear interactions," Phys. Rev. C90, 061306 (2014).

[273] Sven Binder, Joachim Langhammer, Angelo Calci, and Robert Roth, "Ab Initio Path to Heavy Nuclei," Phys. Lett. B 736, 119-123 (2014).

[274] A. Ekström, G. R. Jansen, K. A. Wendt, G. Hagen, T. Papenbrock, B. D. Carlsson, C. Forssén, M. HjorthJensen, P. Navrátil, and W. Nazarewicz, "Accurate nuclear radii and binding energies from a chiral interaction," Phys. Rev. C 91, 051301 (2015).

[275] K. Hebeler, S. K. Bogner, R. J. Furnstahl, A. Nogga, and A. Schwenk, "Improved nuclear matter calculations from chiral low-momentum interactions," Phys. Rev. C 83, 031301 (2011).

[276] R. F. Garcia Ruiz et al., "Unexpectedly large charge radii of neutron-rich calcium isotopes," Nature Phys. 12, 594 (2016).

[277] K. Hebeler, H. Krebs, E. Epelbaum, J. Golak, and R. Skibinski, "Efficient calculation of chiral threenucleon forces up to $\mathrm{N}^{3} \mathrm{LO}$ for ab initio studies," Phys. Rev. C 91, 044001 (2015).
[278] C. Drischler, A. Carbone, K. Hebeler, and A. Schwenk, "Neutron matter from chiral two- and three-nucleon calculations up to $\mathrm{N}^{3}$ LO," Phys. Rev. C 94, 054307 (2016).

[279] H. Kummel, K. H. Luhrmann, and J. G. Zabolitzky, "Many-Fermion theory in expS- (or coupled cluster) form," Phys. Rept. 36, 1-36 (1978).

[280] Bogdan Mihaila and Jochen H. Heisenberg, "Ground state correlations and mean field in ${ }^{16} \mathrm{O}$. II. Effects of a three-nucleon interaction," Phys. Rev. C 61, 054309 (2000).

[281] G. Hagen, D. J. Dean, M. Hjorth-Jensen, T. Papenbrock, and A. Schwenk, "Benchmark calculations for ${ }^{3} \mathrm{H},{ }^{4} \mathrm{He},{ }^{16} \mathrm{O}$, and ${ }^{40} \mathrm{Ca}$ with ab initio coupled-cluster theory," Phys. Rev. C 76, 044305 (2007).

[282] G. Hagen, T. Papenbrock, D. J. Dean, A. Schwenk, A. Nogga, M. Włoch, and P. Piecuch, "Coupled-cluster theory for three-body hamiltonians," Phys. Rev. C 76, 034302 (2007).

[283] Dean Lee, "Lattice simulations for few- and many-body systems," Prog. Part. Nucl. Phys. 63, 117-154 (2009).

[284] B. S. Pudliner, V. R. Pandharipande, J. Carlson, Steven C. Pieper, and R. B. Wiringa, "Quantum Monte Carlo calculations of nuclei with $A \lesssim 7$," Phys. Rev. C 56, 1720-1750 (1997).

[285] J. E. Lynn, I. Tews, J. Carlson, S. Gandolfi, A. Gezerlis, K. E. Schmidt, and A. Schwenk, "Chiral Three-Nucleon Interactions in Light Nuclei, Neutron- $\alpha$ Scattering, and Neutron Matter," Phys. Rev. Lett. 116, 062501 (2016).

[286] Robert Roth, "Importance Truncation for Large-Scale Configuration Interaction Approaches," Phys. Rev. C 79, 064324 (2009).

[287] P. Maris, J. P. Vary, P. Navrátil, W. E. Ormand, H. Nam, and D. J. Dean, "Origin of the anomalous long lifetime of ${ }^{14}$ C," Phys. Rev. Lett. 106, 202502 (2011).

[288] G. Hagen et al., "Neutron and weak-charge distributions of the ${ }^{48}$ Ca nucleus," Nature Phys. 12, 186-190 (2015).

[289] T. D. Morris, N. Parzuchowski, and S. K. Bogner, "Magnus expansion and in-medium similarity renormalization group," Phys. Rev. C 92, 034331 (2015).

[290] V. Lapoux, V. Somà, C. Barbieri, H. Hergert, J. D. Holt, and S.R. Stroberg, "Radii and Binding Energies in Oxygen Isotopes: A Challenge for Nuclear Forces," Phys. Rev. Lett. 117, 052501 (2016).

[291] S. Okubo, "Diagonalization of hamiltonian and tammdancoff equation," Prog. Theor. Phys 12, 603 (1954).

[292] S.Y. Lee and K. Suzuki, "The effective interaction of two nucleons in the s-d shell," Phys. Lett. B 91, 173 (1980).

[293] Kenji Suzuki and Shyh Yuan Lee, "Convergent theory for effective interaction in nuclei," Prog. Theor. Phys. 64, 2091 (1980).

[294] A. F. Lisetskiy, B. R. Barrett, M. K. G. Kruse, P. Navrátil, I. Stetcu, and J. P. Vary, "Ab initio shell model with a core," Phys. Rev. C 78, 044302 (2008).

[295] G. R. Jansen, A. Signoracci, G. Hagen, and P. Navrátil, "Open $s d$-shell nuclei from first principles," Phys. Rev. C 94, 011301 (2016).

[296] S. R. Stroberg, A. Calci, H. Hergert, J. D. Holt, S. K. Bogner, R. Roth, and A. Schwenk, "A nucleusdependent valence-space approach to nuclear structure," Phys. Rev. Lett. 118, 032502 (2017).

[297] S. R. Stroberg, H. Hergert, J. D. Holt, S. K. Bogner, and A. Schwenk, "Ground and excited states of doubly open-shell nuclei from ab initio valence-space Hamilto- 
nians," Phys. Rev. C 93, 051301 (2016).

[298] B. Alex Brown and W. A. Richter, "New 'USD' Hamiltonians for the sd shell," Phys. Rev. C 74, 034315 (2006).

[299] H. Hergert, "In-Medium Similarity Renormalization Group for Closed and Open-Shell Nuclei," Phys. Scr. 92, 023002 (2017).

[300] Werner Kutzelnigg and Debashis Mukherjee, "Normal order and extended wick theorem for a multiconfiguration reference wave function," J. Chem. Phys. 107, 432-449 (1997).

[301] Debashis Mukherjee, "Normal ordering and a wick-like reduction theorem for fermions with respect to a multideterminantal reference state," Chem. Phys. Lett. 274, 561 - 566 (1997).

[302] E. Epelbaum, H. Krebs, and U. G. Meißner, "Improved chiral nucleon-nucleon potential up to next-to-next-tonext-to-leading order," Eur. Phys. J. A 51, 53 (2015).

[303] R. J. Furnstahl, N. Klco, D. R. Phillips, and S. Wesolowski, "Quantifying truncation errors in effective field theory," Phys. Rev. C 92, 024005 (2015).

[304] B. D. Carlsson, A. Ekström, C. Forssén, D. Fahlin Strömberg, G. R. Jansen, O. Lilja, M. Lindby, B. A. Mattsson, and K. A. Wendt, "Uncertainty analysis and order-by-order optimization of chiral nuclear inter- actions," Phys. Rev. X 6, 011019 (2016).

[305] J. Simonis, K. Hebeler, J. D. Holt, J. Menéndez, and A. Schwenk, "Exploring sd-shell nuclei from twoand three-nucleon interactions with realistic saturation properties," Phys. Rev. C 93, 011302 (2016).

[306] S. J. Freeman and J. P. Schiffer, "Constraining the $0 \nu 2 \beta$ matrix elements by nuclear structure observables," J. Phys. G: Nucl. Part. Phys. 39, 124004 (2012).

[307] B. A. Brown, M. Horoi, and R. A. Sen'kov, "Nuclear Structure Aspects of Neutrinoless Double- $\beta$ Decay," Phys. Rev. Lett. 113, 262501 (2014).

[308] Amand Faessler, G. L. Fogli, E. Lisi, V. Rodin, A. M. Rotunno, and F. Šimkovic, "QRPA uncertainties and their correlations in the analysis of $0 \nu \beta \beta$ decay," Phys. Rev. D 79, 053001 (2009).

[309] Amand Faessler, G. L. Fogli, E. Lisi, V. Rodin, A.M. Rotunno, and F. Šimkovic, "Overconstrained Estimates of Neutrinoless Double Beta Decay Within the QRPA," J. Phys. G 35, 075104 (2008).

[310] Frank F. Deppisch and Jouni Suhonen, "Statistical analysis of beta decays and the effective value of $g_{A}$ in the proton-neutron quasiparticle random-phase approximation framework," Phys. Rev. C 94, 055501 (2016).

[311] J. Dobaczewski, W. Nazarewicz, and P. G. Reinhard, "Error estimates of theoretical models: a guide," J. Phys. G: Nucl. Part. Phys. 41, 074001 (2014). 